\title{
1 Geologic Mapping of Vesta
}

3 R.A. Yingst (1), S.C. Mest (1), D.C. Berman (1), W.B. Garry (1), D.A. Williams (2), D.

4 Buczkowski (3), R. Jaumann (4), C.M. Pieters (5), M.C. De Sanctis (6), A. Frigeri (6), L. Le

5 Corre (7), F. Preusker (4), C.A. Raymond (8), V. Reddy (7), C.T. Russell (9), T. Roatsch

6 (4), and P.M. Schenk (10)

7 (1) Planetary Science Institute, Arizona, (2) Arizona State University, Arizona, (3) JHU-APL, Maryland; (4)

8 DLR, Institute of Planetary Research, Berlin, Germany, (5) Brown University, Rhode Island, (6) National

9 Institute of Astrophysics, Italy, (7) Max Planck Institute for Solar System Research, Germany, (8) NASA

10 JPL, California Institute of Technology, California, (9) UCLA, California, (10) LPI, Texas (yingst@psi.edu /

11 Fax: +1-920-465-2376)

12

13 Corresponding author: R. Aileen Yingst; yingst@psi.edu; 920-360-3627; 1700 E. Ft.

14 Lowell, Suite 106, Tucson, AZ 85719.

15

16 Submitted: 12 February 2013

17

18 Key words: Dawn, Vesta, geologic mapping

19 


\section{Abstract}

We report on a preliminary global geologic map of Vesta, based on data from the

23 Dawn spacecraft's High-Altitude Mapping Orbit (HAMO) and informed by Low-Altitude

24 Mapping Orbit (LAMO) data. This map is part of an iterative mapping effort; the

25 geologic map has been refined with each improvement in resolution. Vesta has a

26 heavily-cratered surface, with large craters evident in numerous locations. The south

27 pole is dominated by an impact structure identified before Dawn's arrival. Two large

28 impact structures have been resolved: the younger, larger Rheasilvia structure, and the

29 older, more degraded Veneneia structure. The surface is also characterized by a system

30 of deep, globe-girdling equatorial troughs and ridges, as well as an older system of

31 troughs and ridges to the north. Troughs and ridges are also evident cutting across, and

32 spiraling arcuately from, the Rheasilvia central mound. However, no volcanic features

33 have been unequivocally identified. Vesta can be divided very broadly into three

34 terrains: heavily-cratered terrain; ridge-and-trough terrain (equatorial and northern);

35 and terrain associated with the Rheasilvia crater. Localized features include bright and

36 dark material and ejecta (some defined specifically by color); lobate deposits; and mass-

37 wasting materials. No obvious volcanic features are evident. Stratigraphy of Vesta's

38 geologic units suggests a history in which formation of a primary crust was followed by

39 the formation of impact craters, including Veneneia and the associated Saturnalia

40 Fossae unit. Formation of Rheasilvia followed, along with associated structural

41 deformation that shaped the Divalia Fossae ridge-and-trough unit at the equator.

42 Subsequent impacts and mass wasting events subdued impact craters, rims and 
43 portions of ridge-and-trough sets, and formed slumps and landslides, especially within

44 crater floors and along crater rims and scarps. Subsequent to the formation of

45 Rheasilvia, discontinuous low-albedo deposits formed or were emplaced; these lie

46 stratigraphically above the equatorial ridges that likely were formed by Rheasilvia. The

47 last features to be formed were craters with bright rays and other surface mantling

48 deposits. Executed progressively throughout data acquisition, the iterative mapping

49 process provided the team with geologic proto-units in a timely manner. However,

50 interpretation of the resulting map was hampered by the necessity to provide the team

51 with a standard nomenclature and symbology early in the process. With regard to

52 mapping and interpreting units, the mapping process was hindered by the lack of

53 calibrated mineralogic information. Topography and shadow played an important role

54 in discriminating features and terrains, especially in the early stages of data acquisition.

\section{1. Introduction}

$58 \quad$ Geologic mapping is a comprehensive investigative process that organizes

59 disparate datasets into geologic units with the goal of revealing the underlying geologic

60 processes and placing those processes into a global, contextual framework. The arrival

61 of the Dawn spacecraft at the asteroid Vesta provides a first opportunity for this

62 approach to be utilized for Vesta at the sub-km scale, at which features such as impact

63 craters, local landslides and tectonic structures can be resolved. The inner main belt

64 asteroid Vesta is a particularly compelling target for this traditional investigative

65 process because of long-standing evidence for its basaltic surface and longitudinal 
66 mineralogic heterogeneity gathered first through Earth-based polarimetric and

67 spectroscopic measurements [Degewij et al., 1979; Gaffey, 1997, 1983; McCord et al.,

68 1970; Reddy et al., 2010]. Such a surface indicated a differentiated crust and, potentially,

69 volcanic activity in Vesta's past.

70 Prior to the arrival of the Dawn spacecraft, the highest-resolution images of the

71 surface of Vesta (38 km/pxl) were provided by the Hubble Space Telescope (HST; Li et

72 al., 2010). During favorable approach conditions in 1994 and 1996, the HST provided

73 reflectance data at $0.439,0.673,0.953$ and $1.042 \mu \mathrm{m}$, and from these data, albedo,

74 elevation and mineralogical data were derived, from which maps of mineralogic

75 composition and lithology were produced [Binzel et al., 1997; Gaffey, 1997; Li et al.,

$762008,2006]$. These data revealed a surface dominated by regionally distinct units

77 interpreted to be impact-excavated pyroxene-rich plutonic material, results that agreed

78 generally with mineralogic maps created from Earth-based spectroscopy [Degewij et al.,

79 1979; Gaffey, 1997, 1983; Reddy et al., 2010]. Though necessarily generated from

80 images with a resolution no better than 38.5 - $52 \mathrm{~km} / \mathrm{pxl}$ [Binzel et al., 1997; Li et al.,

81 2010, 2008; Zellner et al., 1997], these maps represented first steps in understanding

82 Vesta's geologic history.

83 NASA's Dawn spacecraft entered Vestan orbit on July 16, 2011, and spent one

84 year in orbit to characterize its geomorphology, elemental and mineralogical

85 composition, topography, shape, and internal structure before departing to asteroid

86 Ceres on September 5, 2012. Three orbital phases of the mission returned images at

87 successively higher resolutions; the highest of these was $20-25 \mathrm{~m} /$ pixel. Preliminary

88 geologic results from the initial orbital phase ("Survey orbit") are reported by Russell et 
90 During the pre-encounter phase of the mission, the Dawn science team followed

91 the recommendations of Batson [1990] for planetary geologic mapping and divided the

92 asteroid into 15 quadrangles for geologic mapping. Preliminary global geologic maps

93 were also produced in an iterative fashion as new data became available [Yingst et al.,

$942012 ; 2011]$. These iterations of the global geologic map were utilized by the science

95 team during the active phases of the mission to inform evolving hypotheses, correlate

96 crater size-frequency statistics, mineralogic data and other products with preliminary

97 geologic units, and place new data within a baseline geologic context. This work

98 represents the compilation and analysis of these iterative efforts.

100 2. Approach

101 A geologic map is a visual representation of the distribution and sequence of

102 rock types and other geologic information. It allows observations to be organized and

103 represented in an intuitive format, unifies observations of heterogeneous surfaces

104 made at different localities into a comprehensive whole, and provides a framework for

105 science questions to be answered. A geologic map defines boundaries for the extent and

106 overlap of important characteristics such as mineralogy, topography, morphology and

107 elemental abundance. This information can then be used to analyze relationships

108 between these characteristics; this, in turn, can inform models of thermal and structural

109 evolution. In the case of Vesta, a geologic map also would allow the HED (howardite,

110 eucrite and diogenite) meteorites (a family of meteorites believed to have originated

111 from Vesta [Binzel and Xu, 1993; Consolmagno and Drake, 1977; McCord et al. 1970] and 
112 discussed in more detail in Section 3) to be placed in geologic context, should the

113 sources be located.

114 The goals in creating any geologic map determine the level of detail at which the

115 map is created, and thus the required spatial resolution of data selected for the base

116 map. Where the goal is to summarize the current state of knowledge for a region for

117 archiving, the presented map will differ from one where the purpose is to provide a

118 preliminary overview of geologic context in a setting where data collection is in process,

119 or where the amount or type of data available varies across the mapped region. These

120 latter maps are often iterative - that is, multiple versions are created because each

121 iteration is refined as data become available. An example of such a situation is the

122 geologic mapping that may occur during field work, where a sketch map of local units or

123 layers is created first to inform the choice of future sampling locations, and is updated

124 as those samples are collected and analyzed. The more comprehensive geologic map is

125 generated later, when all the available data has been acquired, refined and analyzed in

126 detail.

127 An orbital mission to another planetary body is analogous to this scenario of field

128 work followed by data analysis, where time in the field mirrors the period of spacecraft

129 data acquisition. A detailed geologic map is often generated after the mission ends, once

130 all the data are acquired and have been fully calibrated and refined. However, as in field

131 work, analysis of data begins as soon as it is acquired. Iterative mapping is a process

132 that provides the geologic context for, and reveals the interrelationships of, geologic

133 characteristics revealed by each emerging dataset. Further, it can do so within a

134 timeframe that allows the map to inform data analysis of other team members on the 
135 mission timeline.

136 The global geologic maps presented here demonstrate the progression of lessons

137 learned from generating each iteration [Yingst et al., 2012; 2011]. Where possible, we

138 have referred back to units and surface features identified by mapping efforts that

139 predate the Dawn mission [Binzel et al., 1997; Gaffey et al., 1997]; we note that because

140 the spatial resolution available for these mapping efforts was $\sim 500$ times coarser than

141 that available here, there are previously named and mapped regions that are not

142 included because they do not exist as geologically defined features. This includes Olbers

143 Regio, identified in HST images as a dark ovoid region approximately $200 \mathrm{~km}$ across.

144 For the final iteration of the map we include type examples of units and landforms, and

145 descriptions and interpretations of primary units; we also attempt to deconvolve and

146 interpret the basic stratigraphy in a relative sense utilizing stratigraphic relationships.

147 We intend for the map to provide a contextual framework for more advanced

148 compositional and/or geomorphological mapping at large scales (smaller regions).

149 However, because the process of data analysis is still in its early stages as of this

150 writing, we expect the concepts for the units and structures presented to evolve as

151 analysis and understanding mature. Our goals for this work are thus twofold: firstly, to

152 provide the community with a preliminary assessment of the geology of Vesta, using

153 traditional geologic mapping methods as a primary tool to perform this assessment;

154 and secondly, to report on and analyze the mapping process as it was conducted during

155 an active mission, where iterative products were fed directly to the team to inform

156 subsequent data acquisition and analysis. 


\section{3. Geologic Setting}

Vesta is an ellipsoidal asteroid with dimensions estimated at $286.3 \times 278.6 \mathrm{x}$

$161223.2 \pm 0.1 \mathrm{~km}$ [Russell et al., 2012]. Efforts by Binzel et al. [1997], Gaffey [1997] and Li

162 et al. [2010; 2008] utilized Earth-based and HST spectral data to identify and interpret

163 low-resolution albedo patterns on the surface. Spectral signatures were also identified

164 based on ground-based spectroscopy and HST Wide-Field Planetary Camera (WFPC2)

165 images that resolved Vesta at up to $\sim 9^{\circ} / \mathrm{pxl}$. Characteristics used to discriminate

166 between potential units included derived albedo, spectral shape, and variations in the

167 depth, position and width of the $1 \mu \mathrm{m}$ absorption band ( $\mathrm{Fe}^{2+}$, a common component of

168 basaltic minerals, has a $1 \mu \mathrm{m}$ absorption band). The lithologic maps derived from

169 analysis of these data showed a surface composed of several discrete, spectrally-similar

170 regions. The hemisphere that Binzel et al. [1997] noted as their "western hemisphere"

171 was interpreted as relatively uniform, similar to iron-rich pyroxenes, and comparable

172 to surface basalts such as eucrite meteorites. By contrast, their "eastern hemisphere"

173 was more diverse, with magnesium-rich pyroxenes and several regions of olivine-rich,

174 diogenite, and low-Ca eucrite regions located near the prime meridian [Binzel et al.,

175 1997; Gaffey, 1997]. The average surface of Vesta was noted as analogous to a mix of

176 howardite or polymict eucrite; these are regolith-derived members of the HED

177 meteorites [Gaffey, 1997]. These results indicated that Vesta has an old, differentiated

178 surface, with spectrally-distinct regions that can be geochemically tied to the HED

179 meteorites. Crystallization ages measured by radiometric dating for HEDs in Earth-

180 based laboratories document that rocks comprising Vesta were formed within the first 
181100 million years of solar system history (4.43-4.55 Gyr [Lugmair and Shukolyukov,

182 1998; Nyquist et al., 1997; Tera et al., 1997; see review by McSween et al., 2011]). The

183 mapping results reveal surface features formed by processes that must postdate the

184 very old age of the HEDs.

185 Data available prior to the Dawn mission indicated that impact cratering was the

186 dominant process on the surface of Vesta (e.g., Gaffey [1997]), and the Dawn data

187 confirm that Vesta has a heavily-cratered surface (e.g., Marchi et al. [2012a]). The south

188 pole is dominated by a large impact structure identified before Dawn's arrival [Thomas

189 et al., 1997a, b]; the name Rheasilvia has been approved by the International

190 Astronomical Union (IAU) for this structure (the IAU is the organization that certifies

191 the nomenclature of planetary features; only IAU-approved names are used throughout

192 this manuscript). The surface also has three large systems of troughs and ridges: one

193 around the equator, one confined to the northern hemisphere, and one cutting across,

194 and spiraling arcuately from, the Rheasilvia central mound [Jaumann et al., 2012]. A

195 color relief map of the surface is shown in Figure 1.

196 Global geologic mapping and image analysis of Vesta using data of increasing

197 resolution acquired at successive phases of the Dawn mission has enabled the

198 identification of several impact basins hundreds of kilometers in diameter (e.g.,

199 Rheasilvia, Veneneia), an ancient heavily cratered northern hemisphere, and regional

200 sets of graben and ridge-and-groove structures (e.g,. Divalia Fossae and Saturnalia

201 Fossae). We have also mapped and characterized a number of geologic units associated

202 with impact basins and craters, regional cratered plains and highlands units, and

203 surficial deposits suggesting localized mass wasting of loose material. 


\section{4. Data and Mapping Procedure}

Each of Dawn's several orbital phases at Vesta provided increasingly higher

208 spatial resolution data that were integrated into the mapping effort, as summarized in

209 Table 1. Using data from each of these phases as "waypoints," we completed three main

210 iterations of the global map. The first was created during the approach phase, when

211 data were taken to determine the Vestan pole (Rotational Characterization, or RC) and

212 to support navigation (Optical Navigation, or OpNav). The RC/OpNav map was based on

213 clear filter data from the Framing Camera (FC), which covered the surface at 3-9

$214 \mathrm{~km} /$ pixel resolution. The second iteration was completed subsequent to Survey orbit

215 and was based on FC clear filter data at $\sim 200 \mathrm{~m}$ resolution and a Digital Terrain Model

216 (DTM) derived from Survey orbit image data [Jaumann et al., 2012; Preusker et al.,

217 2012]. The third was based on data from the High-Altitude Mapping Orbit (HAMO) with

218 a spatial resolution of $\sim 61 \mathrm{~m} /$ pixel. For a summary of the navigational aspects of the

219 Dawn at Vesta mission, including orbit tracks, see Russell et al. [2005] and Polanskey et

220 al. [2011].

221 The preparation of all iterations of the geologic map followed the methods

222 developed and described by Shoemaker and Hackman [1962], Wilhelms [1990, 1972],

223 Tanaka et al. [2010] and Greeley and Batson [1990]. Units were defined on the basis of

224 characteristics such as morphologic features, surface textures, color and albedo; where

225 color is defined as the color ratio scheme used in Clementine multispectral images

226 (Clementine data are often displayed as ratios of $415 / 750 \mu \mathrm{m}$ in red, $750 / 950 \mu \mathrm{m}$ in 
227 green, and 750/415 $\mu \mathrm{m}$ in blue; here we use $440 / 750 \mu \mathrm{m}, 750 / 920 \mu \mathrm{m}$ and 750/440 $228 \mu \mathrm{m})$.

229 For the first iteration (RC/OpNav), the portion of the surface imaged was 230 mapped in its entirety by four separate workers who then compared and consolidated

231 results. This method was adopted for two reasons: Firstly, it was important in this early 232 stage to allow all mappers to become familiar with the surface features as rapidly as 233 possible, as the first map had to be produced within a few months. Secondly, we wanted 234 to calibrate the different approaches that each mapper utilized, so that in later 235 iterations this would be a relatively known factor.

236 For the second iteration, each worker mapped one of four broad regions: $30-90^{\circ}$

$237 \mathrm{~S}$, or $0-120^{\circ}, 120-240^{\circ}$ or $240-360^{\circ}$ longitude, with each of the latter three blocks

238 ranging from $30^{\circ} \mathrm{S}$ latitude to the limit of coverage in the north. Some overlap occurred

239 where features or units straddled these longitudinal blocks; this overlap allowed the

240 mappers to compare results and address any potential areas of disagreement. For the

241 third iteration, each worker mapped a different one of these longitudinally-defined

242 blocks, to lessen bias and to allow each worker to become familiar with the geology of

243 the entire body at significantly higher resolution than revealed by RC/OpNav.

\section{4.1. Iterative mapping}

246 4.1.1. RC/OpNav iteration. The initial iterations of the map, based on FC images

247 from the OpNav and Survey phases of the mission, were generated in Adobe Illustrator

248 by the mapping team. The map produced from RC and OpNav data is shown in Figure 2.

249 This map covers latitudes from $0--90^{\circ}$, however, $\mathrm{RC} / \mathrm{OpNav}$ data covered some areas 
250 further north than the equator. Units are informed by these more northern images.

251 Hypotheses to be tested in this iteration were that the surface of Vesta would show

252 evidence of two processes: (1) cratering, especially in the form of a large crater at or

253 near the south pole; and (2) volcanism, in the form of some surface features, possibly

254 vents and flows (as predicted by Wilson and Keil [1996]), or pyroclastic material.

255 In this iteration of the map, we divided the surface into brighter- and darker-

256 toned units, as well as circular features that were inferred at the time (and later

257 confirmed) to be impact craters. Large variations in topography were also visible,

258 including several prominent scarps. Other features identified included the south polar

259 impact crater (Rheasilvia) and two ridges identified in later iterations as the scarp

260 discontinuously bounding the crater (ridge1and ridge2 in Figure 2); lower-albedo

261 regions associated with Rheasilvia ejecta (d1); a high plateau (BT2, Vestalia Terra); and

262 grooves within the Rheasilvia impact structure (noted as GHT on Figure 2, with

263 individual troughs outlined where they could be identified). Prominent features on

264 Vesta that could not be discerned at this resolution included Veneneia and all other,

265 smaller craters. The large topographic variation from the top of Vestalia Terra to the

266 bottom of the craters Marcia, Calpurnia and Minucia was noted but we mapped it

267 incorrectly in this iteration as a crater rim (the eastern rims of the three craters

268 combined) and a potential complex crater peak (the high-standing rim of Marcia and

269 Calpurnia, which were among the highest albedo features seen at this resolution; see

270 Figure 3). This combination of features likely represents what was originally identified

271 as the dark albedo feature "Olbers Regio" on previous maps, as noted by Reddy et al.

272 [2012]. Notably, though the ridge-and-trough system around the equator is very 
273 prominent, it was not identified or mapped at this resolution (the northern latitudes

274 were not imaged at this altitude). The walls of the deepest troughs, which could be seen

275 in some of the earliest FC images, were mapped as curved crater rims. In terms of color

276 ratio data (defined as FC monochrome data and color ratio data, where red-blue tones

277 capture the visible continuum and green tones capture the relative strength of the

278 ferrous absorption band at $1.0 \mu \mathrm{m}$ ), the most prominent feature noted was a deposit

279 that appears orange-toned. This feature was first identified in the images shown in

280 Figure 3, and inferred to be associated with a nearby circular feature (confirmed as an

281 impact crater in Survey data, and named Octavia). Its location is broadly similar to that

282 of a potential olivine signature noted by Binzel et al. [1997]. In this iteration we mapped

283 the deposit as a surface mantling feature, pending more detailed morphologic data. We

284 note, however, that another prominent "orange" toned deposit that was revealed in the

285 Survey data around the crater Oppia does not correlate with any spectral signature

286 noted by Binzel et al. [1997]. These types of deposits are discussed in more depth in 287 section 5.5.1.

288 The geologic map in Figure 2 was used to assist the team in highlighting and

289 preparing for the types of features and terrains that would be encountered as the

290 mission progressed. Outstanding issues to be addressed included: understanding the

291 unique nature of Rheasilvia (whether there was a large extent of impact melt; why there

292 was a central mound rather than a peak or an inner/outer ring complex; the processes

293 that formed the ridge-trough complex); confirming circular features as impact craters

294 and thus beginning to deconvolve the cratering history and relative age of the surface;

295 clarifying the scale and extent of features so they could be compared to similar features 
296 on other bodies; and characterizing the nature of the higher- and lower-albedo regions, 297 especially in relation to topography. The data also confirmed some important previous 298 observations, including the presence of the large south-polar impact structure [Thomas 299 et al., 1997a, b].

300 4.1.2. Survey iteration. The geologic map resulting from Survey data is shown in 301 Figure 4. For this second iteration, all basemap products were created using a 302 coordinate scheme developed by the science team (known informally as the "Claudia" 303 system after a crater at $0^{\circ}$ longitude). Our goal at this point was to facilitate the 304 mapping process, which was most easily done by using the products created by team 305 members assigned to that work. We thus used this team-derived coordinate system as 306 shown in Figures 4-22. Note that at the time of this writing, the PDS is providing Dawn 307 data in a longitude system that can be obtained from the Claudia longitude by 308 subtracting $150^{\circ}$.

309 FC continued to be the basemap, but improved color coverage and preliminary

310 VIR data were also available. Though the interpretation of VIR band combinations is not

311 clear as of this writing, we include the data because it informed some boundaries and

312 interpretations. The geologic map was compiled in ArcGIS software (v. 10.1) using

313 digital mapping techniques as outlined in the NASA Planetary Geologic Mapping

314 Handbook [Tanaka et al., 2010]. We chose to utilize ArcGIS instead of continuing to use

315 Adobe Illustrator because digital mapping facilitates unit characterizations, feature

316 correlation, and crater counts. This iteration of the map was produced at 1:1,000,000 317 scale. 
At this resolution and coverage, we were able to identify and describe the gross

319 characteristics of many of the main geologic units on Vesta (cratered plains and

320 cratered highlands; equatorial and northern trough terrains; equatorial cratered

321 terrain; bright and dark ejecta and crater materials; and Rheasilvia mound and ridge-

322 and-groove terrain). First estimates of crater size-frequency distribution were

323 calculated based on those units [Marchi et al., 2012c; Schmedemann et al., 2012]. We

324 confirmed our observations from the previous iteration that no large melt sheet

325 associated with Rheasilvia exists on the surface. Other crater features were identified

326 and mapped with this iteration, including: craters with higher-albedo ejecta rays;

327 craters with lower-albedo ejecta rays; and so-called "bimodal" craters with one rim

328 portion sharp and the other more degraded [Krohn et al., 2012].

329 Major craters other than Rheasilvia were identified. The largest of these was the

330 Veneneia impact structure, lying north of the younger Rheasilvia crater and identified

331 by sections of rim scarp associated with a semi-circular topographic low. The

332 interpretation of this set of features as an impact structure was vigorously debated

333 among the mappers and the greater Dawn science team. Arguments against such an

334 interpretation included: (a) the shape of the proposed impact structure was not

335 circular and had an irregular vertical profile; (b) the center of the topographic low did

336 not correspond precisely with the center of the proposed structure; and (c) the rim

337 scarp was not continuous. The presence of Veneneia was not confirmed until the

338 acquisition of HAMO data, which revealed that the rim scarp was more complete than

339 had previously been believed. 
341 continuous structural features rather than as scattered grooves or as disconnected

342 peaks and valleys. These features' dimensions were mapped (northern cratered trough

343 terrain and equatorial ridge and trough terrain respectively) and measured, and

344 potential correlations with other terrains were assessed. Specifically, the two large

345 ridge-and-trough complexes outside the south polar region were preliminarily

346 associated with tectonic disruptions that occurred during the formation of Rheasilvia

347 and Veneneia impact structures (equatorial and northern respectively] [Buczkowski et

348 al., 2012; Jaumann et al., 2012].

349 Remaining issues at this stage of the iterative mapping process included

350 clarifying the nature of the higher- and lower-albedo regions, and their relation to

351 stratigraphy and topography.

352 4.1.3. HAMO iteration. For this iteration, shown in Figure 5, we used a

353 monochrome (clear filter) FC mosaic as our basemap. Images in this mosaic have an

354 average spatial scale of $\sim 70 \mathrm{~m} /$ pixel for HAMO. This base was imported into ArcGIS and

355 supplemented by the Survey DTM. FC color ratio images from Survey orbit with a

356 spatial scale of $\sim 250 \mathrm{~m} /$ pixel and Visible and InfraRed (VIR) hyperspectral images from

357 the Survey and HAMO orbits with spatial scales of 700 and $200 \mathrm{~m} /$ pixel, respectively,

358 provided information on surface composition and were used to refine unit boundaries.

359 The final map in Figure 5 was produced at a scale of 1:500,000.

360 In mapping this iteration, we expanded the list of units into a more traditional

361 Description of Material Units (DOMU). Names were assigned to each unit, some 
362 associated with IAU-compliant names of the most prominent or characteristic feature 363 associated with that unit [Roatsch et al., 2012].

364 With this iteration, the data was sufficient to resolve differences in surface

365 texture down to the $\sim 100 \mathrm{~m}$ scale, and a different illumination angle allowed albedo

366 differences to be more clearly discerned through comparison to global Survey data. For

367 this iteration, we mapped craters down to $2 \mathrm{~km}$ diameter at the request of the Dawn

368 science team (for clarity, only craters $>6 \mathrm{~km}$ diameter are shown on Figure 5). Using

369 this information we were able to make several improvements to the geologic map,

370 including (1) differentiating some larger units into smaller ones based on relative crater

371 density and texture; (2) mapping the extent of Rheasilvia-modified terrain, which

372 extends to nearly the equator in some places; (3) characterizing the extent of fine-

373 textured ejecta materials at fresher craters; and (4) identifying and analyzing the

374 characteristics of unique small-scale (tens of $\mathrm{m}$ ) features such as units with lobate

375 boundaries, and pitted terrain within crater floors. With regard to differentiation of

376 larger units, we used improved surface texture information to divide portions of the

377 Rheasilvia ridge-and-groove terrain (Figure 4) into Rheasilvia smooth material (less-

378 heavily cratered, smoother than surrounding Rheasilvia materials) and the more

379 heavily cratered highlands and cratered plains north of it (Rs, cp and ch in Figure 5

380 respectively). Units added included mass wasting material and Rheasilvia smooth

381 material. We also revisited the boundaries of cratered terrain (cratered highlands and

382 cratered plains in earlier maps) based on roughness of texture and relative crater

383 density. Specifically, the area represented by cratered highlands increased at the

384 expense of cratered plains. Additionally, the boundaries of the cratered highlands unit 
385 were expanded at the expense of ridge-and-trough terrain, to include areas with similar 386 mean topography and FC color. The equatorial ridge-and-groove terrain and northern

387 cratered trough terrain were renamed the Divalia Fossae ridge-and-trough and

388 Saturnalia Fossae cratered trough units, as the improved resolution revealed that

389 grooves (a more general term) were indeed troughs.

390

391 5. Material Units

392

Based on data from the Dawn instruments, the surface of Vesta is comprised of

394 four major terrains: individual craters and associated impact materials, widespread

395 undifferentiated cratered units, the Saturnalia and Divalia Fossae units, and materials

396 associated with the Rheasilvia impact structure. Other more localized units include

397 lobate, smooth and tholus materials, and mass-wasting materials. We describe each of

398 these units in terms of morphology, surface texture, relative crater density, topography

399 and color ratio data. We also present type localities for each unit; images are from

400 HAMO unless otherwise noted. The symbology and nomenclature used are shown in

$401 \quad$ Figure 5.

402

403 5.1. Surficial deposits

404

405 5.1.1. Mass wasting material (mw)

406 5.1.1.1. Description: This unit takes the form of deposits along the bases of steep

407 slopes or crater walls due to mass movement of material, indicating the mobility of the 
408 regolith [Jaumann et al., 2012; Pieters et al., 2012]. There are several morphologies

409 represented within this unit. Slumps occur as sequences of benches separated by

410 crescent-shaped cliffs or scarps beginning at the top of a slope (Figure 6a). Lobate or

411 fan-shaped, smooth-textured deposits also occur, often associated with impact craters

412 (Figure 6b). More irregularly bounded deposits tend to have a hummocky texture and

413 often display subtle or more diffuse boundaries.

414 5.1.1.2. Interpretation: We interpret this unit as debris falls, slumps or slides

415 formed through slope failure that may be associated with a number of possible

416 processes that involve mass movement of material downslope. Possible drivers include

417 "seismic" shaking associated with impact crater formation or slope failure due to

418 overburden. The team requested that these materials be mapped together by process

419 rather than associated feature, in order to facilitate analysis of their distribution and

420 thus how regolith mobility may vary by location.

421

\section{$422 \quad$ 5.1.2. Bright lobate (bl)}

423 5.1.2.1. Description: This unit is characterized by lobes that extend from crater

424 rims or local topographic highs (e.g., scarps) onto crater floors or local topographic

425 lows (e.g., Figure 7). Deposits that fall within this unit have a convex-up topography,

426 with lobate margins and smooth to hummocky surfaces. Deposits differ from mw

427 deposits in their convex, positive topography and relatively sharp boundaries. Bright

428 lobate deposits have intermediate albedo in FC monochrome images, with a yellow tone

429 in color ratio images, though the color is not unique to this unit. These deposits

430 generally have lower crater abundances compared to their surroundings. 
432 material, younger than the surrounding surface. These are likely flow deposits and may

433 be the result of either impact ejecta flow lobes or impact debris transported downslope

434 as mass movement. Yellow areas in color ratio images tend to be smoother and may be

435 composed of impact melts.

\section{5.1.3. Dark lobate (dl)}

438 5.1.3.1. Description: Dark lobate deposits (Figure 8) are similar in morphology to 439 the bright lobate unit but have a distinctively lower albedo. These materials extend 440 from crater rims or scarps onto crater floors or local topographic lows. Deposits have a

441 flat topography, with lobate margins and relatively smooth surfaces. Deposits differ 442 from mw deposits in their flat surfaces, very smooth textures and relatively sharp 443 boundaries. Dark lobate deposits have low albedo in FC monochrome images, with a 444 yellow tone in color ratio images, though again, this color is not unique to this unit.

445 These deposits generally have lower crater abundances compared to their 446 surroundings.

447 5.1.3.2. Interpretation: Similarly to the bright lobate unit, we interpret the dark 448 lobate unit to be impact-derived material; surface texture is consistent with impact melt 449 [McCord et al., 2012; Reddy et al., 2012b].

\section{$451 \quad$ 5.1.4. Smooth unit (s)}

452 5.1.4.1. Description: The smooth material unit is highly localized, consisting of 453 several exposures found on the floor and rim of Marcia crater (Figure 9). This unit 
454 displays overall smooth, dark and relatively featureless surfaces at the tens of meters

455 scale, except for variable amounts of small impact craters and some clusters of pits.

456 Smooth unit deposits have low albedo in FC monochrome images, and are typically

457 blue-green to green-brown in FC color ratio images.

458 5.1.4.2. Interpretation: We interpret this smooth unit to be very young impact

459 melt. Pit clusters are associated with low hydrogen and OH levels [De Sanctis et al.,

460 2012; Prettyman et al., 2012] and have been interpreted as pits formed when volatiles

461 from a volatile-rich impactor boiled off subsequent to crater formation [Denevi et al.,

462 2012]. Mapping results are consistent with this hypothesis. Alternately, the unit could

463 be fine-grained materials deposited by mass movement.

$465 \quad$ 5.1.5. Tholus ( $t)$

466 5.1.5.1. Description: There are two tholi mapped at this resolution: Aricia and

467 Lucaria Tholi (Figure 10). These are defined as isolated topographic highs with heavily-

468 cratered surfaces and dark lobate patches associated with them. They have

469 intermediate albedos in FC monochrome images and appear dark blue to purple in FC

470 color ratio images.

471 5.1.5.2. Interpretation: We interpret the tholus unit to be impact-sculpted crust,

472 possibly containing volcanic dikes or intrusions, or volcanic cones. Dark-rayed crater

473 material and dark lobate patches on Aricia Tholus may indicate basaltic material

474 exposed by impact cratering. Alternatively, the dark-rayed crater could have an

475 exogenic source (i.e., carbonaceous meteorite), and the small lobate patches could be

476 impact ejecta flows or impact melts [Reddy et al., 2012b]. 


\section{$478 \quad$ 5.2. Cratered terrains}

\section{$479 \quad$ 5.2.1. Cratered highlands (ch)}

480 5.2.1.1. Description: This extensive unit has a heavily-cratered surface and a

481 higher albedo and overall topography than the surrounding plains (Figure 11). The

482 boundary between this and other units is occasionally subtle but discernable as a

483 combination of steepening topographic slope and an increase in roughness of surface

484 texture. This unit is concentrated along the equator and includes the Vestalia Terra

485 high. An intermediate albedo is seen in monochrome FC images, with localized bright

486 and dark patches. In FC color ratio images color ranges from purple-red to blue tones.

487 Early analysis of VIR data led to an interpretation of the spectral signature of this unit 488 as having howarditic mineralogy [De Sanctis et al., 2012].

489 5.2.1.2. Interpretation: We interpret this unit to be ancient terrain. The Vestalia 490 Terra region may be a preserved section of ancient crustal materials (e.g., Raymond et 491 al. [2013]) or a mound of accumulated ejecta, modified by later impact cratering, 492 producing a distinctive topographic high. Because the surface texture of cratered 493 highlands is similar throughout, showing no distinct difference between the higher 494 Vestalia Terra and the surrounding cratered highlands, the former interpretation is 495 currently preferred. If this is the case, Vestalia Terra may be the oldest terrain on Vesta.

$497 \quad$ 5.2.2. Cratered plains (cp)

498 5.2.2.1. Description: The cratered plains unit (Figure 12) occurs as narrow, 499 somewhat isolated regions of smoother, topographically lower, relatively sloping 
500 terrain with lower crater density than the Divalia Fossae unit. All occurrences of this

501 unit are bounded by cratered highlands, the Divalia Fossae unit or both.

502 5.2.2.2. Interpretation: We interpret this unit to be ancient cratered terrain

503 degraded or smoothed by either the emplacement of a thinning layer of Rheasilvia

504 ejecta, or the degradation of sloping material over time.

505

506 5.3. Crater material

507

508 Impact cratering is an important geologic process on Vesta. Craters are also

509 probes into previous geologic conditions because they excavate into lower layers.

510 Crater morphology shows many similarities to other small, airless, rocky bodies. Small,

511 fresh craters are characterized by sharp-crested, narrow rims and bowl shapes, while

512 larger fresh craters have flat floors and may display slumping of rim walls, finer-

513 textured floor fill, or visible ejecta material (craters 1- $3 \mathrm{~km}$ in diameter are of

514 indeterminate state of degradation because morphology is below resolution, and are

515 mapped as points). Degraded craters have subdued but distinct, continuous rims and

516 varying shapes. Enclosed sub-circular or ovoid depressions also exist; these have lower

517 topography than the surroundings but lack a discernable rim or bowl shape. Complex

518 craters are characterized by high-topography rugged hills and arcuate scarps forming

519 partial rings. We interpret the state of degradation to be generally correlated with

520 crater age, with the least degraded craters being the youngest and the most degraded

521 being the oldest; however, differences in the rheology of target materials may affect the

522 shape of the crater. Some sharp-rimmed craters show degraded rim sections (bi-modal 
523 craters). The process that forms such craters is unclear. One hypothesis is that such

524 craters were formed on a slope, with the upslope side being more susceptible to mass-

525 wasting (e.g., Krohn et al. [2012]); many, but not all, such craters fit this model.

526 There are several types of crater materials that show unique features that

527 provide clues to underlying layers. These are enumerated below.

528

\section{$529 \quad$ 5.3.1. Bright crater ray material (bcr)}

530 5.3.1.1. Description: This material forms halos, streaks or patches radiating from

531 relatively fresh impact craters, extending to or beyond one crater diameter (Figure 13).

532 These rays are very thin, as the underlying topography, and in some cases, the

533 underlying texture, are visible. Additionally, opacity decreases with increasing distance

534 from the source. Bright crater ray material displays high albedo in monochrome FC

535 images, and a bright white to yellow color in FC color ratio images.

536 5.3.1.2. Interpretation: We interpret this high albedo material to be mantling

537 impact ejecta excavated from deeper layers, analogous to lunar crater rays. Lunar rays

538 are considered the youngest features on the Moon (e.g., Dietz [1946]); we infer from

539 superposition relationships (rays are stratigraphically the highest feature) that bright

540 crater rays are relatively young features on Vesta, not yet affected by space weathering

541 [Pieters et al., 2012].

542

\section{$543 \quad$ 5.3.2. Dark crater ray material (dcr)}

544 5.3.2.1. Description: Dark crater ray material (Figure 14) forms low-albedo radial

545 halos, streaks or patches that mantle underlying material and extend to or beyond one 
546 crater diameter. Rays often are associated with craters in which thin (100-500m thick),

547 discrete layers of dark material are visible in the crater walls; craters that have dark

548 crater ray material often occur in clusters. The distribution of these rays around craters

549 is often asymmetrical and the underlying topography and texture are visible. Opacity

550 decreases with increasing distance from the source. Dark crater rays display low albedo

551 in monochrome FC images and a dark purple color in Clementine-type FC color ratio

552 images.

$553 \quad$ 5.3.2.2. Interpretation We interpret this low albedo ray material to be impact

554 ejecta excavated from thin, dark, discontinuous subsurface layers whose boundaries

555 can be inferred by the presence of these dark crater rays; alternatively, rays could

556 consist of low albedo material dispersed from dark, possibly carbonaceous impactors.

557 We infer from superposition relationships that dark crater rays are relatively young

558 features.

559

$560 \quad$ 5.3.3. Bright crater material (bc)

561 5.3.3.1. Description: Bright crater material (Figure 15) is characterized by a

562 continuous, high-albedo ejecta blanket, and pronounced continuous crater rims

563 elevated relative to surrounding materials.

564 5.3.3.2. Interpretation: We interpret bright crater material to be high-albedo,

565 relatively fresh and unmodified crater deposits - including rim, ejecta and some floor

566 materials - emplaced by impact processes.

567

568 5.3.4. Dark crater material (dc) 
570 albedo ejecta blankets associated with pronounced continuous crater rims elevated

571 relative to surrounding materials (Figure 16). Most parent craters display dark layers

572 exposed in their interior walls.

573 5.3.4.2. Interpretation: We interpret dark crater material to be similar to craters

574 with dark crater ray materials, where ejecta is inferred to be excavated from dark,

575 discontinuous subsurface layers.

576

\section{5.3.5. Undifferentiated ejecta material (uc)}

578 5.3.5.1. Description: Ejecta material that is continuous and relatively smooth, but

579 has no other discriminating characteristics or clear stratigraphic placement (Figure 17),

580 is mapped as ejecta material, undifferentiated. Pronounced continuous crater rims

581 elevated relative to surrounding materials tend to be associated with such ejecta

582 deposits. Some deposits display a more distinctive color (in the FC color ratio mosaic)

583 compared to underlying terrain; specifically, colors in between those of the bright ejecta

584 (yellows to yellow-green) and dark ejecta (dark violet), thus violet-red to green-blue.

585 5.3.5.2. Interpretation: We interpret this material to be relatively fresh and

586 unmodified, well-preserved impact crater deposits.

587

$588 \quad$ 5.4. Ridge-and-trough terrain

589

$590 \quad$ Linear structural features were discovered on the Martian moon Phobos decades 591 ago in Viking orbiter imagery; Thomas and Veverka [1979] suggested that these grooves 
592 were most likely the result of the large impact that formed Stickney crater.

593 Furthermore, they predicted that similar lineaments would be observed on other small

594 cratered bodies, since craters formed in the laboratory [e.g., Fujiwara and Asada, 1983]

595 and on planets [e.g., Reimold et al., 1998] tend to display radial fractures. This

596 prediction was realized when images of asteroids began to be returned to Earth. A

597 number of different types of linear structural features -including grooves, fractures,

598 troughs and ridges - have been observed on a number of asteroids, including Gaspra

599 [Ververka et al., 1994], Ida [Sullivan et al., 1996], Itokawa [Sasaki et al., 2006], Eros

600 [Prockter et al., 2002; Buczkowski et al., 2008], Steins [Keller et al., 2010], Lutetia

601 [Sierks et al., 2011; Thomas et al., 2012] and now Vesta [Jaumann et al., 2012;

602 Buczkowski et al., 2012].

603 While the ridges and troughs that characterize these terrains are likely the result

604 of intense faulting of pre-existing terrain, we mapped the material as separate units

605 because the crust has been disrupted to such an extent that it is impossible to identify

606 the characteristics of the pre-existing terrain, to classify it.

607

608 5.4.1. Saturnalia Fossae material (Sf)

609 5.4.1.1. Description: The Saturnalia Fossae unit is found in the northern

610 hemisphere. Topographically lower than surrounding units, the unit consists of heavily-

611 cratered terrain cross-cut by NW-SE trending degraded ridges and flat-floored troughs.

612 The largest, most prominent of these troughs is Saturnalia Fossa, at widths up to 39.2

$613 \mathrm{~km}$, depths up to $4.0 \mathrm{~km}$ and a length of at least $458 \mathrm{~km}$; a trough to the south of Lepida

614 crater is potentially a continuation of the fossa [Buczkowski et al., 2012], which could 
615 mean its length is as great as $640 \mathrm{~km}$. This potential continuation is much deeper than

616 the average fossae $(\sim 6 \mathrm{~km})$, perhaps due to reactivation during the Rheasilvia impact.

617 Associated troughs have widths that range from $\sim 5$ to $\sim 15 \mathrm{~km}$, lengths of $\sim 20$ to $\sim 140$

$618 \mathrm{~km}$, and depths of $50 \mathrm{~m}$ to $\sim 2.0 \mathrm{~km}$. The fossae cut terrain containing the highest crater

619 density observed on Vesta (e.g., Figure 18). The unit is intermediate albedo in FC

620 monochrome images, and blue-purple in FC color ratio images.

621 5.4.1.2. Interpretation: We interpret the Saturnalia Fossae unit to be Vestan

622 crustal material, modified into fault-bounded graben by impact and tectonic processes

623 [Buczkowski et al., 2012]. Fault-plane analysis suggests that northern troughs and

624 grooves of the Saturnalia Fossae may be some form of tectonic response to formation of

625 the Veneneia impact structure (pre-Rheasilvia). This unit represents one of the oldest

626 on Vesta.

627

628 5.4.2. Divalia Fossae material (Df)

629 5.4.2.1. Description: The Divalia Fossae unit is characterized by terrain cut by

630 prominent ridges and deep, flat-floored troughs trending parallel to the equator (Figure

631 19). Trough widths range from $10-22 \mathrm{~km}$, lengths are $90-465 \mathrm{~km}$, and depths are $1-2$

$632 \mathrm{~km}$; the largest is Divalia Fossa, at $465 \mathrm{~km}$ length and width ranging from 14.5-21.8

$633 \mathrm{~km}$. Numerous craters superpose the troughs. This unit does not have a unique color in

634 FC monochrome or color ratio images. It displays intermediate crater density, and is

635 stratigraphically younger than the Rheasilvia Formation and Saturnalia Fossae unit.

636 5.4.2.2. Interpretation: Like the Saturnalia Fossae unit, we interpret the Divalia

637 Fossae unit to consist of Vestan crustal material, heavily modified into fault-bounded 
638 graben by impact and tectonic processes. In the case of the Divalia Fossae, formation of

639 this sequence of ridges and troughs [Buczkowski et al., 2012; Jaumann et al., 2012] is

640 consistent with interpretation that they formed by tectonic response to formation of the

641 Rheasilvia impact crater.

642

643 5.5. Rheasilvia Formation

644

$645 \quad$ 5.5.1. Rheasilvia smooth terrain (Rs)

646 5.5.1.1. Description. Much of the outer boundary of the Rheasilvia impact crater is

647 defined by Rheasilvia smooth terrain (Figure 20). This unit occurs as irregularly-

648 bounded regions of very smooth material at lower resolution, often located on slopes or

649 topographically lower regions. This unit has a slightly lower albedo than the cratered

650 plains and the Divalia Fossae unit to the north. Crater density is moderate; clusters of

651 dark-rayed craters often occur within the confines of this unit.

652 5.5.1.2. Interpretation. We interpret this smooth member to be ejecta emplaced

653 during the Rheasilvia impact event, potentially modified later through mass movement

654 of material.

655

656 5.5.2. Rheasilvia ridge-and-groove terrain ( $\mathrm{Rrg})$

657 5.5.2.1. Description. The Rheasilvia floor is characterized by curvilinear ridges

658 and grooves that are kilometers to tens-of-kilometer long (Figure 21). There are two

659 pronounced trends: one running sub-radially from the Rheasilvia central mound, and

660 one paralleling the curvilinear scarps that bound Rheasilvia. Lobate deposits appear 
661 downslope of some steep sloped ridges and mantle underlying cratered terrain. Crater

662 density appears lower than Vestalia Terra, Divalia Fossae, and Saturnalia Fossae.

663 Rheasilvia Formation ridge-and-trough terrain appears blue-green in FC color ratio 664 images.

665 5.5.2.2. Interpretation. We interpret this member to be Rheasilvia material,

666 heavily modified by post-impact processes. Impact materials form the floor of the

667 Rheasilvia impact structure and are derived from the Rheasilvia impact event [Schenk et

668 al., 2012]. It is not clear how much of these impact materials are comprised of actual

669 impact melt. Lobate deposits along steep scarps and ridges are interpreted to be slump

670 or other mass wasting deposits, driven by overburden.

671

672 5.5.3. Rheasilvia mound terrain ( $\mathrm{Rm})$

673 5.5.3.1. Description: The center of Rheasilvia is characterized by an irregular

674 topographic high bisected by a discontinuous scarp. This central mound (Figure 22) has

675 a relatively low crater density between $90-180^{\circ}$ longitude; crater density is higher

676 elsewhere. The surface is granular-textured and smoother than the crater floor. In

677 several areas pre-existing topography can be seen as rugged ridges surrounded by

678 younger surface materials. These ridges appear to be aligned with the larger trending

679 ridges and grooves. Shallow lineaments on the surface mirror this trend across the

680 mound. Lobate deposits ( $\mathrm{mw}$ ) occur along the base of the scarp.

681 5.5.3.2. Interpretation: We interpret this member to be comprised of materials

682 that form the broad central peak of Rheasilvia. Central peaks form by the rebound of the

683 impact point, excavating rocks from depth; the composition of the mound may thus be a 
684 probe into the composition of the Vestan subsurface. The scarp boundary appears

685 unstable, as evidenced by the presence of mw deposits. Crater density is interpreted to

686 be lower because craters have been degraded, obscured or destroyed by slope failure.

687 Smaller ridges surrounded by younger surface materials are interpreted to be

688 extensions of the Rheasilvia ridge-and-groove member (Rrg) partially buried and

689 subdued by mw material.

690

691 5.6. Surface Features

692

693 Vesta has distinctive features that overlie several of the geologic units noted

694 here. These features differ from the geologic units defined above because they mantle

695 but do not cross-cut, deform, or otherwise alter the underlying units. Such features are

696 shown as symbols on the map.

697

698 5.6.1. Dark Mantling Material

699 This feature is diffuse ejecta material of a unique spectral signature, mantling

700 underlying topography. This material is localized in several patches, the largest of

701 which is the type feature associated with the Oppia crater ejecta blanket. This feature is

702 visible as a low albedo mantle in monochrome images, more clearly identified as

703 brown-toned in FC "true" color images, and orange-toned in FC stretched color images.

704 It is potentially correlated with a 2.8 micron feature in VIR data [De Sanctis et al., 2012].

705 Like most ejecta, this features displays decreasing opacity with increasing distance from

706 the source. The mantle is continuous and has thickness, and we have therefore mapped 
707 it as a unit, though it clearly "mantles" material lying below it. We interpret this feature

708 to be diffuse impact ejecta of unique composition or physical properties, potentially

709 excavating a layer of distinctive mineralogy or lithology.

710

$711 \quad$ 5.6.2. Catena

712 Catena appear as linear, overlapping or chevron-shaped clusters of similar

713 diameter craters. We interpret catena to be chains of secondary craters associated with

714 the formation of a larger impact crater.

715

716 6. Relative Stratigraphy

717 We used superposition relationships, crater frequency, and cross-cutting

718 relationships to determine a preliminary stratigraphic sequence for Vesta. The oldest

719 coherent unit is cratered highlands material, a unit that includes the high-standing

720 Vestalia Terra. This unit is the lowest stratigraphic unit and is the best candidate for

721 representing Vesta's ancient, primary crust, if it still exists. Stereo topography [Preusker

722 et al., in review] and geophysical data [Raymond et al., 2013] suggests that Vestalia

723 Terra is not only a topographic high but also has both a positive gravity and density

724 anomaly, suggesting it is a remnant of Vesta's original crust.

$725 \quad$ Cratered plains have fewer craters, a smoother texture, and are lower

726 topographically, than cratered highlands. They are thus interpreted to have formed

727 later. It is currently not clear whether the few troughs within the cratered plains are

728 associated with Divalia Fossae or the Rheasilvia Formation, or formed by other

729 processes; nor is it entirely clear whether they cross-cut this unit, or are truncated by it. 
730 Unlike the cratered highlands, cratered plains' smoother texture may indicate mantling

731 by some ejecta from the Rheasilvia impact. An alternate interpretation for this

732 smoother texture is that because these isolated "islands" are on downsloping terrain,

733 the terrain has been smoothed through slumping or degradation through mass

734 movement.

735 The Saturnalia Fossae unit cross-cuts the cratered highlands and plains and thus

736 must post-date these units, but is cross-cut by the Divalia Fossae unit and is therefore

737 stratigraphically older than that unit. The poles of the planes described by the graben in

738 this unit are oriented around a point that describes the central floor of Veneneia,

739 indicating that the Saturnalia Fossae were formed through fracturing associated with

740 the Veneneia impact [Buczkowski et al., 2012; Jaumann et al., 2012].

741 The Rheasilvia Formation cross-cuts (in the case of the ridges and grooves) or

742 stratigraphically superposes (in the case of ejecta material), cratered highlands and

743 plains. While the extent of the units in this formation do not reach far enough north to

744 reveal a stratigraphic relationship between it and the Saturnalia Fossae unit, because

745 the Rheasilvia Formation cuts (Rrg) or overlies (Rs) the cratered plains and highlands,

746 but is cut by Divalia Fossae, it must lie stratigraphically between these units. We note

747 also that if the formation of Veneneia drove the fracturing associated with the

748 Saturnalia Fossae, and is overlain by Rheasilvia, then Rheasilvia must post-date the

749 Saturnalia Fossae. The Rheasilvia Formation itself overprinted or obliterated any visible 750 vestige of older units.

751 The Divialia Fossae unit cross-cuts all lower units, except possibly the cratered

752 plains, as noted above. As is the case for Veneneia, the poles of the planes described by 
753 the graben in this unit are oriented around a point that describes the central floor of

754 Rheasilvia. This indicates that the unit is the result of tectonic deformation associated

755 with the Rheasilvia impact (e.g., Buczkowski et al. [2012]; Jutzi and Asphaug [2011]). An

756 alternate hypothesis is that troughs formed due to spin up of Vesta, although the spin

757 up would still be due to the impact.

758 Craters exist at each stratigraphic level; some, such as Marcia, dominate the pre-

759 existing terrain to a great extent. Marcia Crater is a special case, in which the ejecta is

760 unique and the craters themselves overlie all other units except for the ones internal to

761 them $(\mathrm{mw}, \mathrm{t}, \mathrm{dl})$. Marcia is the youngest large $(>25 \mathrm{~km})$ crater on the surface of Vesta,

762 with cratering model age estimates of $\sim 40-160 \mathrm{Ma}$ (Williams et al., in revision). As

763 noted above, craters are mapped and placed stratigraphically based on the level of

764 degradation of rims, as well as infill and slumping. Sharp craters are interpreted to be

765 the youngest craters, while degraded and highly degraded craters and depressions are

766 considered progressively older. Based on inference from the lunar example, we also

767 interpret rayed craters to be very young. Because craters with softened and slightly

768 more subdued morphology than the freshest craters do not have ray systems, either

769 bright or dark, this indicates that surface soil is being altered and such features are

770 being erased over time [Pieters et al., 2012]. Dark-rayed craters lie stratigraphically

771 above the Divalia Fossae unit, but because they tend to cluster in discrete locations and

772 excavate a narrow, low-albedo stratigraphic layer, these crater rays are interpreted to

773 be due to excavation of this dark layer rather than an indicator of age. Again, because

774 these craters do not occur globally, it is not clear where this class of craters lies within

775 the stratigraphic column. 
The many relatively young surficial deposits indicate that portions of Vesta's

777 crust have been eroded or altered more recently. Many of these deposits are associated

778 with specific young craters (e.g. the lobate material, pitted terrain, mass movement in

779 and around Marcia) and the driving force behind their formation is likely associated

780 with the crater. For example, pitted terrain has been hypothesized to have formed

781 through volatile release associated with crater formation [Denevi et al., 2012], while

782 mass movement of material in many cases is likely due to slumping from crater

783 formation or oversteepening. Other mass movement deposits are likely also associated

784 with slumping of oversteepened scarps or other high points. Mass movement events are

785 most likely driven by the seismic shock created by nearby small impacts.

786 The youngest features on Vesta are those mapped surface features where the

787 underlying geologic unit is still visible. The most prominent of these is the dark mantle

788 feature. This feature is of uncertain composition, but its broad extent in several

789 locations suggests the presence of a layer of unique composition that has been

790 excavated in these locations. The presence of this feature is not correlated with

791 topography: Oppia lies at a topographic low, for example, and Vestalia Terra is the

792 highest point on Vesta. There is, however, a correlation with latitude. All craters

793 excavating dark mantling features occur between $30^{\circ}$ and $0^{\circ}$ latitude; none exist within

794 the Rheasilvia Formation. The inference is that either the excavated layer associated

795 with the dark mantling feature was present but was stripped by the Rheasilvia impact,

796 or the layer was never present south of the equator. Alternatively, the source of the

797 material may be exogenic (the impactors) rather than endogenic (Vesta's crust). 
799 telescopic spectral evidence of basaltic mineralogies, and the presence of basaltic

800 minerals and glassy textures that are diagnostic of terrestrial basaltic lava flows found

801 in the HED meteorites (e.g., Binzel et al., 1997; Gaffey, 1997; McCord et al., 1970;

802 McSween et al., 2011). Wilson and Keil $(1997,1996)$ used mathematic and petrologic

803 models to predict the types of volcanic deposits that should occur on Vesta, including

804 surface lava flows a few kilometers to several tens of kilometers in length, channelized

805 flows on steeper slopes, a lack of uniform sheet flows and shield volcanoes, shallow

806 dikes and deep intrusions, and minimal pyroclastic fall deposits. These studies

807 suggested that volcanic features, particularly lobate lava flows, might be resolved on

808 Vesta's surface by Dawn, although such deposits likely would have been heavily

809 disrupted by impact craters and thus not easily recognized. Analysis of HAMO and

810 LAMO images covering $\sim 85 \%$ of Vesta's surface has revealed no unequivocal

811 morphological evidence for volcanic-related flow features on the surface. The lack of

812 discrete volcanic features on Vesta is consistent with the hypothesis, based on analysis

813 of basaltic material in the HED suite, that volcanism on Vesta occurred only during the

814 first 10-100 Ma of Vesta's history (e.g., McSween et al., 2011; Schiller et al., 2010), and

815 that since then impact processes have degraded any ancient volcanic features to the

816 point where they cannot be distinguished.

\section{7. Absolute age-dating based on crater statistics}

819 Crater counting is a common technique used to derive relative and absolute ages

820 for planetary surfaces, based on the principle that the older a surface is, the more 
821 craters it accumulates from impacts [Hartmann, 1966a,b; Hartman and Neukum, 2001].

822 We have not used crater statistics for unit dating; our units are based solely on

823 traditional geologic mapping principles such as cross-cutting relationships. Rather, we

824 present here initial crater statistics that have been derived from the units mapped.

825 In the case of Vesta, counts have been conducted using different methodologies

826 for counting and for determining absolute ages from those counts (e.g., Marchi et al.,

827 this issue; O'Brien et al., this issue; Schmedemann et al., this issue). We note that there is

828 not full consensus on which units can be reliably assessed for absolute age through

829 crater counting statistics using HAMO data. Those units that most workers agree can be

830 reliably dated using larger ( $>10 \mathrm{~km}$ diameter) craters fully resolvable by HAMO include

831 cratered highlands (ch), Rheasilvia ridge-and-groove terrain and Rheasilvia smooth

832 terrain (Rrg and Rs, part of the Rheasilvia Formation), and the Divalia Fossae and

833 Saturnalia Fossae units (Df and Sf), both categorized as ridge-and-trough material. A

834 detailed treatment of absolute age-dating of the surface of Vesta based on crater

835 statistics can be found in Marchi et al. [this issue], O'Brien et al. [this issue] and

836 Schmedemann et al. [this issue].

837 In each method, the diameter of every crater $\geq 10 \mathrm{~km}(\geq 2 \mathrm{~km}$ for this work) for

838 each geologic unit was measured and recorded using ArcGIS tools (e.g., Kneissl et al.

839 [2011]), using the HAMO and LAMO clear filter FC mosaics as a base, along with

840 elevation data from the Gaskell shape model to assist in crater identification.

\section{7.1. Method 1.}


843 In the method utilized by Schmedemann et al. [this issue], and in this work, size-

844 frequency distributions (SFDs) were plotted cumulatively in the Craterstats2 program

845 [Michael and Neukum, 2010]. Absolute ages were determined for counts using the third

846 iteration of the production and chronology functions developed by Schmedemann et al.

847 (this issue). The crater production function for Vesta is based on the lunar production

848 function (derived from measuring craters over different units on the Moon [Neukum

849 and Ivanov, 1994]), which is scaled to impact conditions on Vesta. This technique uses

850 the whole range of observed crater sizes on the well-investigated lunar surface and

851 results in reliable slopes of the Vestan crater distribution, especially at small diameters.

852 The lunar chronology [Neukum and Ivanov, 1994] is scaled to Vestan impact conditions,

853 which are characterized by a factor of about 4 lower impact velocities and about 30

854 times higher impact flux when compared to the Moon.

855 In general, the current iteration of the production function fits well at diameter

856 ranges from $50 \mathrm{~m}$ up to $500 \mathrm{~km}$, except for in areas older than the Rheasilvia impact

857 structure. For these, crater counts between 8 and $\sim 30-50 \mathrm{~km}$ are significantly below the

858 production function. In this size range it is possible that a multitude of different effects

859 may be observed, caused by the formation of Veneneia and Rheasilvia. These effects

860 include multiple resurfacing events on a global scale as well as additional possibly

861 intense but short lived cratering by the Vestan collisional family (vestoids).

862 Schmedemann et al. [this issue] discuss this matter in more detail. These effects are not

863 observed on younger surfaces ( $<2 \mathrm{Ga}$ ) like the current surface of the Rheasilvia

864 Formation, where the observed crater size-frequency distribution closely follows the

865 Vestan crater production function up to $\sim 30 \mathrm{~km}$ crater size. The chronology function 
866 used in this method appears fairly accurate as it gives surface ages of the large units

867 (Rheasilvia, Veneneia, global resurfacing) close to the peaks of the Ar-Ar age probability 868 plot by Bogard [2011].

869

\section{$870 \quad$ 7.2. Method 2 .}

871 An independent assessment of the cratering record of five units

872 of Vesta has been carried out using the crater catalog of Marchi et al. [2012a]. The crater

873 retention ages of these terrains have been derived using the Model Production Function

874 (MPF) [Marchi et al., 2012a-c; this issue]. In this method, the current impact rate on

875 Vesta is computed using a model main belt size-frequency distribution [Bottke et al., 876 2005]. Then, a Pi-group crater scaling law [Holsapple and Housen, 2007] is used to

877 convert the current impact flux in a crater production function. This gives the

878 cumulative number of craters as a function of crater size per unit time and unit surface.

879 Finally, the chronology function derived by $O^{\prime} B r i e n$ et al. [this issue] has been used to

880 model the impact flux at Vesta in the past. The resulting crater SFDs are shown in

881 Figure 23, along with some examples of MPFs.

882

883 7.3. Results and comparison

884 Absolute ages for units as derived by method 1 are shown in Figure 24. We note 885 that individual material units could be characterized by many different formation ages.

886 Here we give average ages for the presented material units from our counts. In some 887 cases, we also include the ages of smaller key areas inside the global units, reported by 
888 Schmedemann et al. [2012; this issue], in order to illustrate the range of ages that have 889 been reported within the material units.

890 The Rheasilvia mound $(\mathrm{Rm})$ terrain yields an average age of $1.78 \pm 0.32 \mathrm{Ga}$.

891 However, the formation age of the Rheasilvia mound may also be estimated by

892 examining a small area on top of the mound. This small area gives a significantly higher 893 age of $3.59(+0.079 /-0.18)$ Ga. The lower average age likely results from repeated mass 894 wasting, activity that did not affect the very top of the central mound significantly. For 895 the Rheasilvia ridge-and-groove terrain (Rrg), we determined an age of $2.03 \pm 0.18 \mathrm{Ga}$, 896 similar to the Rheasilvia smooth terrain (Rs), with an average age of $2.03 \pm 0.11 \mathrm{Ga}$. In 897 the range of Matronalia Rupes and in the eastern part of the Veneneia impact structure 898 the Rs unit has older surface ages, around 3.4 Ga (Veneneia floor: 3.39+0.098/-0.25 Ga) 899 [Schmedemann et al., this issue].

900 The cratered plains (cp) have an average age of $2.98+0.18 /-0.28$ Ga and the 901 Divalia Fossae unit (Df) has an average age of 3.36 \pm 0.03 Ga. For the cratered highlands 902 (ch), we calculated an average age of $3.42 \pm 0.01 \mathrm{Ga}$, and for the Saturnalia Fossae unit 903 (Sf), we calculated an average age of $3.46+0.12 /-0.59 \pm 0.02 \mathrm{Ga}$. A correlation of units is 904 shown in Figure 25.

905 In this work, utilizing a methodology based on Schmedemann et al. [this issue], 906 we counted a similar number of craters as Marchi et al. [this issue]. Differences in ages 907 are due mainly to the use of the different production functions, so we note caveats for 908 both methods here. Counts by Schmedemann et al. [this issue] include additional large 909 craters identified solely by topographic signature, resulting in older ages for some units. 910 The crater SFDs shown in Figure 24 exhibit wavy shapes that differ significantly from 
911 the shape of the crater SFDs for the Rheasilvia floor. They also differ from the shape of

912 the model production function. This could be explained by a past impact SFD

913 significantly different from the present (a hypothesis not supported by crater SFD from

914 other asteroids [Marchi et al., this issue]), or collisional evolution models [Bottke et al.,

915 2005]. Alternately, the geologic units may not define regions with uniform crater

916 retention ages. Finally, the most heavily cratered terrains on Vesta may have reached a

917 state of quasi-equilibrium [Gault, 1970], where newly formed craters have erased

918 significantly older craters. In this case, the cratering retention ages would represent

919 lower limits for the true age of the surface.

920 Though beyond the scope of this work, it is clear that age assessments of the

921 various units and subunits (particularly ch, Df and Sf) need further investigation. In

922 support of that work, we will continue our crater counting efforts with the goal of

923 having a complete crater catalog down to diameters of $500 \mathrm{~m}$.

924

\section{8. Geologic history}

926 A sequence of geologic events is listed below based on the above observations

927 and interpretations. Ages given are those calculated in this work; we refer the reader to

928 Marchi et al. [this issue] and Schmedemann et al. [this issue] for comprehensive crater

929 statistics data and results.

930 1. Differentiation, fractionation and crystallization of a primary crust, $\sim 4$ Ga (this

931 is a time horizon, defining the oldest craters, rather than an absolute date).

932 Formation of the oldest impact craters; this sequence is represented by the

933 cratered highlands and plains, and Vestalia Terra is interpreted to be a remnant 
of this crust. We calculated an age of $\sim 3.36-2.40$ Ga for these units, but note that

935

936

937

938

939

940

941

942

943

944

945

946

947

948 formed or were emplaced. The latest features to be formed were craters with 949 bright rays, and surface features such as dark mantle material. there is a discrepancy between the morphology and superposition relationships that indicate the greater age of cratered highlands and plains compared to the Saturnalia Fossae unit and Veneneia impact structure (3.36 and 2.40 Ga versus 3.38 Ga respectively). We expect the crater statistics for the more northern regions to be refined as these last-acquired images are analyzed.

2. Formation of Veneneia ( $\sim 3.78$ Ga based on Schmedemann et al., this issue) and the Saturnalia Fossae unit, followed by the Rheasilvia impact event $(\sim 3.55$ Ga based on Schmedemann et al., this issue) and associated Divalia Fossae unit. Both events likely resulted in global resurfacing.

3. Subsequent impacts and mass movement events that subdued impact craters, complex crater rims and portions of ridge-and-trough sets, and formed slumps and landslides, especially within crater floors and along rims and scarps. Subsequent to the Rheasilvia Formation, discontinuous low-albedo deposits

\section{9. Lessons learned through the mapping process}

952 The Dawn at Vesta mission has provided a unique opportunity to not only

953 geologically map globally a small, sub-spherical, airless rocky body using high-

954 resolution data, but to record and analyze the mapping process during that mission, to

955 better identify best practices under such circumstances. Lessons learned during this 956 process are reported in this section. 


\section{9.1. Iterative mapping process during data acquisition}

959 Executed progressively throughout data acquisition, the mapping process

960 provided the team with geologic proto-units in a timely manner. Rapid data acquisition,

961 and the consequent need to generate new products quickly, led us to three conclusions.

962 Firstly, though the original plan was for two individuals to conduct the iterative

963 mapping throughout the data acquisition phase of the mission, in execution we chose to

964 divide the labor between four mappers. This plan worked better than two would have

965 done, as products were demanded by the team nearly as quickly as four people could

966 produce them. We thus recommend that similar efforts to create global geologic maps

967 during data acquisition employ at least three or four experienced mappers. We note

968 that for a body of this size, we found the division of labor that we adopted to be effective

969 (one mapper for each pole, and one each for the east and west equatorial regions, with

970 that individual changing between each data acquisition phase).

971 Secondly, we found that experience, both in mapping and in identifying and

972 interpreting the types of features that were present, was a crucial factor in producing

973 rapidly a global geologic map that provided information about coherent units

974 connected by similar process. We found that less experience in the process tended to

975 lead to maps that grouped disconnected features, or to mapping features extraneous to

976 the science questions driving map production. Additionally, the experienced mappers

977 were able to work more quickly by using standard approaches with which they were

978 already familiar, and were able to discuss and debate similarities and difference

979 between mapped regions more efficiently. This efficiency was improved when 
980 discussions of early map drafts included individuals having strong expertise in

981 modeling the types of features we were identifying. The radial scarps in the Rheasilvia

982 impact structure were identified as early as OpNav (Figure 2); similarly, the shape and

983 morphology of the "grooved" terrain was interpreted to be ridges and grooves by team

984 members who were familiar with the processes predicted to create such features, and

985 thus knew what clues to look for to identify and discriminate them from other linear

986 features such as graben, scarps, or crater chains. The ability to draw from a team's

987 broad expertise greatly improved our ability to map accurately and efficiently.

988 Thirdly, we found that the minimal time between data acquisition and product

989 generation meant that overthinking was minimized among the mappers. On the other

990 hand, the pressure upon the global mapping team was enormous to produce precise

991 unit boundaries that fed into other investigations (crater counting statistics, for

992 example, or other mapping projects), as rapidly as possible. This pressure drove the

993 mapping team to retain shortcuts longer than they might have been ideally used. For

994 example, the team retained much of the standardized nomenclature and symbology

995 originally adopted as a preliminary step, rather than allowing unit definitions and

996 nomenclature to evolve more organically with each iteration. We believe that

997 interpretation of the resulting map was hampered by the necessity to provide the team

998 with a standard nomenclature and symbology early in the process, and we might have

999 learned more about the unique features of Vesta if we had been less driven by the

1000 mission timeline to formalize features and symbology quickly. We thus recommend

1001 retaining generic descriptors and symbology for as long as possible into the global

1002 mapping process. 


\section{9.2. Mapping and interpreting units}

1005 The process of mapping and interpreting units was affected by both the 1006 intermittent acquisition of new data and the nature of the data acquired. We noted 1007 three important ways in which this was the case.

1008 Firstly, as detailed in section 4.1, RC/OpNav allowed us to identify some of the 1009 major features on Vesta, though we identified most units and determined most general 1010 boundaries using Survey data. However, there were some changes made to the units 1011 between acquisition of Survey and HAMO data. One notable one was the nature of the 1012 boundaries of Vestalia Terra; it was not clear until HAMO data was acquired, whether or 1013 not the majority of the boundaries around this rise were scarps. Through HAMO, as 1014 well, the mappers debated whether the cratered plains were a discrete unit, and if so, 1015 what their boundaries were and how they could be defined. Of all the units listed in 1016 Figure 5, it is the identification and boundaries of cratered plains of which we are most 1017 unsure, and a more definitive characterization of this unit may require fully calibrated 1018 and interpreted mineralogic data.

1019 Secondly, and related to this issue, the fact that fully calibrated VIR multispectral 1020 data was not available was a hindrance to the mapping process and subsequent 1021 interpretation. On a body like Vesta, where few clues exist to distinguish one cratered 1022 terrain from another besides crater density, this was especially true. Additionally, 1023 without a way to estimate the mineralogy of crater ejecta we could not utilize craters as 1024 probes into the composition of the subsurface. Ultimately we were never able to use 1025 VIR multispectral to define or refine units, only to more specifically describe units. 
1027 derived from other iterations of the map, it was clear that when only coarse resolution

1028 was available, features with a larger topographic range, such as craters, and ridges and

1029 troughs, were easier to identify than other features and terrains. Not every

1030 interpretation based on topography was correct; we interpreted the combined rims of

1031 Marcia, Calpurnia and Minucia (seen in Figure 3) as a single large, individual scarp

1032 (Figure 2). However, topography and shadow played a more important role in correct

1033 identification than morphology, especially where boundaries were raised (e.g., Vestalia

1034 Terra). We believe this is in part because topographic variations are greater on Vesta

1035 than on many previously mapped terrestrial bodies; for example, Vesta's ratio of

1036 surface relief to radius is $\sim 15 \%$, compared to $\sim 1 \%$ for the Moon and Mars [Jaumann et

1037 al., 2012]. Other units, and thus the potential processes from which they stem, were

1038 difficult to identify or interpret based solely on differences in morphology or albedo.

1039 For example, the Rheasilvia smooth member (Rs) and the Rheasilvia ridge-and groove

1040 member (Rrg) were noted in the RC/OpNav map in Figure 2 as D2 and D1 respectively,

1041 but the boundaries were mapped as uncertain and the units themselves were difficult to

1042 interpret. In summary, we believe that for small, irregular bodies, topography is a more

1043 discriminating characteristic than morphology for identifying and characterizing

1044 features and units, especially when the available resolution is very coarse.

1045

1046 Acknowledgements

1047 The authors are grateful to Corey Fortezzo, Trent Hare, Jenny Blue and other

1048 personnel at USGS for their invaluable discussions and for creating the ArcGIS base 
1049 materials that greatly simplified the mapping process. Thanks go especially to the Dawn 1050 operations, engineering and science team. The authors also gratefully acknowledge the 1051 insightful comments of Drs. Jim Zimbelman and Jim Skinner, whose reviews greatly 1052 improved this manuscript. This work was funded through NASA Dawn at Vesta 1053 Participating Scientist Program grant NNX10AR19G to RAY. Italian co-authors were 1054 funded through the Italian Space Agency (ASI) grant 1/004/12/0.

1055

1056 


\section{References}

1058

1059 Batson, R.M., 1990. Map formats and projections used in planetary cartography, in:

1060 Greeley, R., Batson, R.M. (Eds.), Planetary Mapping. Cambridge Univ. Press, pp.

$1061 \quad 266-267$.

1062

1063 Binzel, R.P., Xu, S., 1993. Chips off of asteroid 4 Vesta: Evidence for the parent body of 1064 achondrite meteorites. Science. 260, 186-191.

1065

1066 Binzel, R.P., Gaffey, M.J., Thomas, P.C., Zellner, B.H., Storrs, A.D., Wells, E.N., 1997.

1067 Geologic mapping of Vesta from 1994 Hubble Space Telescope images. Icarus, 128, $106895-103$

1069

1070 Bogard, D.D. (2011), K-Ar ages of meteorites: Clues to parent-body thermal histories, $1071 \quad$ Chemie der Erde - Geochemistry, 71: 3, 207-226.

1072

1073 Bottke, W.F., Durda, D.D., Nesvorny, D., Jedicke, R., Morbidelli, A., Vokrouhlicky, D., 1074 Levison, H., 2005. The fossilized size distribution of the main asteroid belt. Icarus. $1075 \quad 175,111-140$.

1076

1077 Buczkowski, D.L., Wyrick, D.Y., Iyer, K.A., Kahn, E.G., Scully, J.E.C., Nathues, A., Gaskell, 1078 R.W., Roatsch, T., Preusker, F., Schenk, P.M., Le Corre, L., Reddy, V., Yingst, R.A., 1079 Mest, S., Williams, D.A., Garry, W.B., Barnouin, O.S., Jaumann, R., Raymond, C.A., 
1080

1081

1082

1083 Buczkowski, D.L., Barnouin-Jha, O.S., Prockter, L.M., 2008. 433 Eros lineaments: Global

1084

1085

1086 Consolmagno, G.J., Drake, M.J., 1977. Composition and evolution of the eucrite parent 1087

1088

1089

1090

1091

1092

1093 Denevi, B.W., Blewett, D.T., Buczkowski, D.L., Capaccioni, F., Capria, M.T., De Sanctis, 1094 M.C., Garry, W.B., Gaskell, R.W., Le Corre, L., Li, J.-Y., Marchi, S., McCoy, T.J., Nathues, 1095 1096 1097 1098 1099

1100 De Sanctis, M.C., Coradini, A., Ammannito, E., Filacchione, G., Capria, M.T., Fonte, S., 1101 Magni, E., Barbis, A. Bini, A., Dami, M., Ficai-Veltroni, I., Preti, G., the VIR team, 1102 2011. The VIR spectrometer. Space Sci. Rev. 163, 329-369. 
1104 De Sanctis, M.C., Nathues, A., Ammannito, E., Capaccioni, F., Frigeri, A., Le Corre, L.,

1105 Jaumann, R., Palomba, E., Pieters, C.M., Reddy, V., Stephan, K., Tosi, F., Yingst, A.,

1106 Zambon, F., Barucci, M.A., Blewett, D.T., Capria, M.T., Combe, J.-Ph., Denevi, B.W.,

1107 Keller, H.U., Marchi, S., McCord, T.B., McFadden, L.A., McSween, H., Raymond, C.A.,

1108 Russell, C.T., Sunshine, J., Toplis, M., Li, J.-Y., 2012. First mineralogical maps of 4

1109 Vesta, Lunar Planet. Sci. Conf. 43 ${ }^{\text {rd }}$, Abs. \#1902.

1110

1111 Dietz, R.S., 1946. The meteoritic impact origin of the Moon's surface features. Jour. Geol. $111254,359-375$.

1113

1114 Frigeri, A., De Sanctis, M.C., Ammannito, E., Yingst, R.A., Mest, S., Capaccioni, F., Garry, B.,

1115 Magni, G., Palomba, E., Petro, N., Tosi, F., Williams, D., Zambon, F., Jaumann, R., 1116 Pieters, C.M., Raymond, C.A., Russell, C.T., Dawn Team, 2012. Correlation between 1117 preliminary mineralogic and geologic maps of Vesta. Lunar Planet. Sci. Conf. $43^{\text {rd }}$, 1118 Abs. \#2934.

1119

1120 Fujiwara, A. and Asada N., 1983. Impact fracture patterns on Phobos ellipsoids, Icarus. $112156,590-602$.

1122

1123 Gaffey, M. J., 1983. The asteroid (4) Vesta: Rotational spectral variations, surface 1124 material heterogeneity, and implications for the origins of basaltic achondrites. 1125 Lunar Planet. Sci. Conf. 14th, 231-232. 
1127 Gaffey, M.J., 1997. Surface lithologic heterogeneity of Asteroid 4 Vesta. Icarus. 127, 130$1128 \quad 157$.

1130 Gault, D.E., 1970. Saturation and equilibrium conditions for impact cratering on the 1131 lunar surface: Criteria and implications. Radio Sci. 5, 273-291.

1132

1133 Greeley, R., Batson, R.M. (Eds.), 1990. Planetary Mapping, Cambridge Univ. Press, 296 $1134 \quad$ pp.

1135

1136 Hartmann, W.K., 1966a. Martian cratering. Icarus 5, 565-576.

1138 Hartmann, W.K., 1966b. Early lunar cratering. Icarus 5, 406-418.

1140 Hartmann, W.K., Neukum, G., 2001. Cratering chronology and evolution of Mars, in 1141 Chronology and Evolution of Mars (R. Kallenbach, J. Geiss, and W.K. Hartmann, 1142 eds.), Kluwer Press and International Space Science Institute, pp. 165-194.

1143

1144 Holsapple, K.A., Hausen, K.R., 2007. A crater and its ejecta: An interpretation of Deep $1145 \quad$ Impact. Icarus. 187, 345-356.

1146

1147 Jaumann, R., Williams, D.A., Buczkowski, D.L., Yingst, R.A., Preusker, F., Hiesinger, H., 1148 Schmedemann, N., Kneissl, T., Vincent, J.B., Blewett, D.T., Buratti, B.J., Carsenty, U., 
Denevi, B.W., De Sanctis, C.M., Garry, W.B., Keller, H.U., Kersten, E., Krohn, K., Li, J.Y., Marchi, S., Matz, K.D., McCord, T.B., McSween, H.Y., Mest, S.C., Mittlefehldt, D.W., Mottola, S., Nathues, A., Neukum, G., O’Brien, D.P., Pieters, C.M., Prettyman, T.H., Raymond, C.A., Roatsch, T., Russell, C.T., Schenk, P., Schmidt, B.E., Scholten, F., Vesta's shape and morphology. Science. 336, 687-690, doi:10.1126/science.1219122.

1157 Jutzi, M., Asphaug, E., 2011. Mega-ejecta on asteroid Vesta. Geophys. Res. Lett. 38, 1158 doi: 10.1029/2010GL045517.

1160 Keller, H.U. et al., 2010. E-type asteroid (2867) Steins as imaged by OSIRIS on board 1161 Rosetta. Science. 327, 190-193. DOI:10.1126/science.1179559

1162

1163 Kneissl, T., van Gasselt, S., Neukum, G., 2011. Map-projection-independent crater size1164 frequency determination in GIS environments---New software tool for ArcGIS.

$1165 \quad$ Planet. Space Sci. 59, 1243-1254.

1166

1167 Krohn, K., Jaumann, R., Stephan, K., Elbeshausen, D., Preusker, F., Roatsch, T., Otto, K., 1168 Matz, K.D., Raymond, C.A., Russell, C.T., 2012. Unusual bimodal craters on slopes, 1169 Vesta. Europ. Planet. Sci. Conf. 7, Abstract EPSC2012-463-3. 1170

1171 Li J.-Y., McFadden, L.A., Parker, J.Wm., Young, E.F., Stern, S.A., Thomas, P.C., Russell, C.T., 
Sykes, M.V., 2006. Photometric analysis of 1 Ceres and surface mapping from HST observations. Icarus. 182, 143-160.

1174

1175

1176

1178

1179

1180

1181 1182

1183

1184 1185

1186

1190 Marchi, S., Massironi, M., Vincent, J.-B., Morbidelli, A., Mottola, S., Marzari, F., Kuppers, 1191 M., Besse, S., Thomas, N., Barbieri, C., Naletto, G., Sierks, H., 2012b. The cratering history of asteroid (21) Lutetia. Planet. Space Sci. 66, 87-95. 
1194 Marchi, S., McSween, H. Y., O'Brien, D., Schenk, P., De Sanctis, M.C., Gaskell, R., Hiesinger, H., Jaumann, R., Mottola, S., Preusker, F., Raymond, C. A., Roatsch, T., Russell, C.T., Yingst, R.A., 2012c. Vesta collisional history revealed by Dawn: Building a Vesta global crater catalog. Lunar Planet. Sci. Conf., 43 ${ }^{\text {rd }}$. Absract \#1617.

1198

1199

1200 1201 1202 1203 1204 1205 1206 1207 1208 1209

1210 McSween, H.Y., Jr., Mittlefehldt, D.W., Beck, A.W., Mayne, R.G., McCoy, T.J., 2011. HED 1211 1212 1213

1214 Michael, G.G., Neukum, G., 2010. Planetary surface dating from crater size-frequency 1215 distribution measurements: Partial resurfacing events and statistical age 1216 uncertainty. Earth Planet. Sci. Lett. 294, 223-229. 
1218 Neukum, G., Ivanov, B.A., 1994. Crater size distributions and impact probabilities on 1219 Earth from lunar, terrestrial-planet, and asteroid cratering data, in: Gehrels, T., 1220 Matthews, M.S., Schumann, A. (Eds.), Hazards due to Comets and Asteroids, Space 1221 Science Series, University of Arizona Press, Tucson, AZ., p. 359.

1222

1223 Nyquist, L.E., Bogard, D., Takeda, H., Bansal, B., Wiesmann, H., Shih, C.-Y., 1997.

1224 Crystallization, recrystallization, and impact-metamorphic ages of eucrites $1225 \quad$ Y792510 and Y791186. Geochim. Cosmochim. Acta. 61, 2119-2138.

1226

1227 Pieters, C.M., Ammannito, E., Blewett, D.T., Denevi, B.W., De Sanctis, M.C., Gaffey, M.J., Le 1228 Corre, L., Li, J.-Y., Marchi, S., McCord, T.B., McFadden, L.A., Mittlefehldt, D.W., 1229 Nathues, A., Palmer, E., Reddy, V., Raymond, C.A., Russell, C.T., 2012. Distinctive 1230 space weathering on Vesta captures regolith mixing processes. Nature. 1231 doi:10.1038/nature11534.

1232

1233 Polanskey, C.A., Joy, S.P., Raymond, C.A., 2011. Dawn science planning, operations and 1234 archiving. Space Sci. Rev. 163, 511-543.

1235

1236 Prettyman, T.H., Feldman, W.C., McSween, H.Y., Jr., Dingler, R.D., Enemark, D.C., Patrick, 1237 D.E., Storms, S.A., Hendricks, J.S., Morgenthaler, J.P., Pitman, K.M., Reedy, R.C., 1238 2011. Dawn's Gamma Ray and Neutron Detector. Space Sci. Rev. 163, 371-459. 
1240 Prettyman, T.H., Mittlefehldt, D.W., Yamashita, N., Lawrence, D.J., Beck, A.W., Feldman,

1241

1242

1243

1244

1245

1246 Preusker, F., Scholten, F., Matz, K.-D., Jaumann, R., Roatsch, T., Raymond, C.A., Russell, 1247

1248 1249

1250 1251 1252 1253 1254 1255 1256 1257

1258 Reddy, V., Gaffey, M.J., Kelley, M.S., Nathues, A., Li, J.-Y., Yarbrough, R., 2010. 1259 1260 1261 1262 Reddy, V., Nathues, A., Le Corre, L., Sierks, H., Li, J.-Y., Gaskell, R., McCoy, T., Beck, A.W., 
Schroeder, S.E., Pieters, C.M., Becker, K.J., Buratti, B.J., Denevi, B., Blewett, D.T., Christensen, U., Gaffey, M.J., Gutierrez-Marques, P., Hicks, M., Keller, U., Maue, T., Mottola, S., McFadden, L.A., McSween, H.Y., Mittlefehldt, D., O’Brien, D.P., Raymond, C., Russell, C., 2012a. Color and albedo heterogeneity of Vesta from Dawn. Science. 336, 700-704.

Reddy, V., Le Corre, L., O’Brien, D. P., Nathues, A., Cloutis, E.A., Durda, D.D., Bottke, W.F., Bhatt, M.U., Nesvorny, D., Buczkowski, D., Scully, J.E.C., Palmer, E.M., Sierks, H., Mann, P.J., Becker, K.J., Beck, A.W., Mittlefehldt, D., Li, J.-Y., Gaskell, R., Russell, C.T., Gaffey, M.J., McSween, H.Y., McCord, T.B., Combe, J.-P., Blewett, D., 2012b. Delivery of dark material to Vesta via carbonaceous chondritic impacts. Icarus. 221, 544-559.

Reddy, V., Li, J.-Y., Le Corre, L., Scully, J.E.C., Gaskell, R., Russell, C.T., Park, R.S., McFadden, L.A., Raymond, C., Nathues, A., Gaffey, M.J., Sierks, H., Becker, K.J., Thangjam, G.S., Comparing Dawn, Hubble Space Telescope, ground-based interpretations of Vesta, in preparation.

Reimold, W.U., Brandt D., Koeberl C., 1998. Detailed structural analysis of the rim of a large complex impact crater: Bosumtwi Crater, Ghana. Geology. 26, 543-546.

Roatsch, T., Kersten, E., Matz, K.-D., Preusker, F., Scholten, F., Jaumann, R., Raymond, C.A., Russell, C.T., 2012. High resolution Vesta High Altitude Mapping Orbit 
(HAMO) Atlas derived from Dawn framing camera images. Planet. Space Sci. 73, 283-286.

1289 Russell, C.T., Raymond, C.A., Fraschetti, T.C., Raymond, M.D. Polanskey, C.A., Schimmels, 1290 K.A., Joy, S.P., 2005. Dawn mission and operations. Proc. IAU Symp. 229, doi:10.1017/S1743921305006691.

1292

1293 Russell, C.T., Raymond, C.A., Coradini, A., McSween, H.Y., Zuber, M.T., Nathues, A., De 1294 Sanctis, M.C., Jaumann, R., Konopliv, A.S., Preusker, F., Asmar, S.W., Park, R.S., 1295 Gaskell, R., Keller, H.U., Mottola, S., Roatch, T., Scully, J.E.C., Smith, D.E., Tricarico, 1296 P., Toplis, M.J., Christensen, U.R., Feldman, W.C., Lawrence, D.J., McCoy, T.J., 1297 Prettyman, T.H., Reedy, R.C., Sykes, M.V., Titus, T.N., 2012. Dawn at Vesta: Testing the protoplanetary paradigm. Science. 684-686, doi:10.1126/science.1219122.

1300

1301 Sasaki, S. and 27 others, 2006. Observations of 25143 Itokawa by the Asteroid 1302 Multiband Imaging Camera (AMICA) of Hayabusa: Morphology 1303 of brighter and darker areas. Lunar Planet. Sci. Conf. 37th, Abstract 1671.

1305 Schenk, P., O’Brien, D.P., Marchi, S., Gaskell, R., Preusker, F., Roatsch, T., Jaumann, R., 1306 Buczkowski, D., McCord, T., McSween, H.Y., Williams, D.A., Yingst, A., Raymond, 1307 C., Russell, C.T., 2012. The giant Rheasilvia impact basin, the evolution of asteroid 1308 4 Vesta and its link to meteorites. Science. 336, 694-697. 
1310 Schiller, M., Baker, J.A., Bizzarro, M., 2010. 26Al-26Mg dating of asteroidal magmatism in 1311 the young solar system. Geochim. Cosmochim. Acta. 74, 4844-4864.

1312

1313 Schmedemann, N., Kneissl, T., Ivanov, B., Michael, G., Wagner, R., Neukum, G., Ruesch, O., 1314 Hiesinger, H., Krohn, K., Roatsch, T., Sierks, H., Jaumann, R., Reddy, V., Nathues, A., 1315 Raymond, C.A., Russell, C.T., 2012. Crater size-frequency distribution (CSFD) and 1316 chronology of Vesta — crater counts matching HED Ages. Lunar Planet. Sci. Conf. $1317 \quad$ 43rd, Abstract \#2544.

1318

1319 Shoemaker, E.M., Hackman, R.J., 1962. Stratigraphic basis for a lunar time scale, in: Z. Kopal, Mikhailov, Z.K. (Eds.) The Moon, Internat. Astron. Union Symposium 14, Academic Press, London, UK, p. 289-300.

1322

1323 Sierks, H. et al., 2011. Images of Asteroid 21 Lutetia: A remnant planetesimal from the 1324 early solar system. Science. 334, 487-490.

1325

1326 Sierks, H. Keller, H.U., Jaumann, R., Michalik, H., Behnke, T., Bubenhagen, F., Buttner, I., 1327 Carsenty, U., Christensen, U., Enge, R., Fiethe, B., Gutierrez Marques, P., Hartwig, 1328 H., Kruger, H., Kuhne, W., Maue, T., Mottola, S., Nathues, A., Reiche, K.-U., 1329 Richards, M.L., Roatch, T., Schroder, S.E., Szemerey, I., Tschentscher, M., 2011. 1330 The Dawn Framing Camera. Space Sci. Rev. 163, 263-327. 
1332 Sullivan R., Greeley, R., Pappalardo, R., Asphaug, E., Moore, J.M., Morrison, D., Belton,

1333

1334

1335

1336

1337

1338

1340

1341

1342 Tanaka, K.L., Skinner, J.A., Jr., Hare, T.M., 2010. The Planetary Geologic Mapping

1343

1344

1345 Tera, F., Carlson, R.W., Boctor, N.Z., 1997. Radiometric ages of basaltic achondrites and

1346 their relation to the early history of the solar system. Geochim. Cosmochim. Acta.

1347

1348

1349 Thomas P., Veverka, J. , 1979. Grooves on asteroids: A prediction. Icarus. 40, 394-405.

1350

1351 Thomas, N. et al., 2012. The geomorphology of (21) Lutetia: Results from the OSIRIS

1352 imaging system onboard ESA's Rosetta spacecraft. Planet. Space Sci..

1353

doi:10.1016/j.pss.2011.10.003.

M.J.S., Carr, M., Chapman, C.R., Geissler, P., Greenberg, R., Granahan, J., Head, J.W.,

Kirk, R., McEwen, A., Lee, P., Thomas, P.C., Veverka, J., 1996. Geology of 243 Ida.

Icarus. 120, 119-139.

Tanaka, K.L., Moore, H.H., Schaber, G.G., Chapman, M.G., Stofan, E.R., Campbell, D.B., Davis, P.A., Guest, J.E., McGill, G.E., Rogers, P.G., Saunders, R.S., Zimbelman, J.R., 1994. The Venus Geologic Mappers Handbook. USGS Open File Rpt. 94-438, 66pp. Handbook. USGS Open File Rpt., 21pp. $61,1713-1731$. 
1355 Thomas, P.C., Binzel, R.P., Gaffey, M.J., Storrs, A.D., Wells, E.N., Zellner, B.H., 1997a.

1356

1357

1358

1359

1360

1361

1362

1363

1364

1365

1366

1367

1368

1369

1370

1371

1372

1373

1374

1375

Impact excavation on asteroid 4 Vesta: Hubble Space Telescope results. Science. $277,1492-1495$.

Thomas, P.C., Binzel, R.P., Gaffey, M.J., Storrs, A.W., Wells, E., Zellner, B., 1997b. Vesta: Spin pole, size and shape from HST images. Icarus. 128, 88-94.

Wilhelms, D.E., 1972. Geologic mapping of the second planet. U.S.Geol.Surv. Interagency Report, Astrogeology. 55.

Wilhelms, D.E., 1990. Geologic mapping, in: Greeley, R., Batson, R.M. (Eds.), Planetary Mapping. Cambridge Univ. Press, New York, pp. 208-260.

Wilson, L. and Keil, K., 1996. Volcanic eruptions and intrusions on asteroid 4 Vesta. J. Geophys. Res. 101, 18,927-18,940.

Wilson, L. and Keil, K., 1997. The fate of pyroclasts produced in explosive eruptions on the asteroid 4 Vesta. Meteoritics Planet. Sci. 32, 813-823.

Yingst, R.A., Williams, D.A., Garry, W.B., Mest, S.C., Petro, N.E., Buczkowski, D., Schenk, P., Jaumann, R., Pieters, C.M., Roatsch, T., Preusker, F., Nathues, A., Le Corre, L., Reddy, V., Russell, C.T., Raymond, C.A., De Sanctis, M.C., Ammannito, E., Filacchione, G., 2011. A preliminary global geologic map of Vesta based on Dawn Survey orbit data. Proc. Of American Geophys. Union. Abstract P43B-0248. 
1376 Yingst, R.A., Mest, S., Garry, W.B., Williams, D.A., Berman, D.C., Jaumann, R., Pieters, C.M., Ammannito, E., Buczkowski, D.L., De Sanctis, M.C., Frigeri, A., Le Corre, L., Preusker, F., Raymond, C.A., Reddy, V., Russell, C.T., Roatsch, T., Schenk, P.M., Dawn Team, 2012. A preliminary global geologic map of Vesta based on highaltitude mapping orbit data. Lunar Planet. Sci. Conf. 43 ${ }^{\text {rd }}$ Abstract \#1359.

1381

1382 Zellner, B., Storrs, A. W., Wells, E., Binzel, R.P., Thomas, P.C., Gaffey, M.J., 1997. Hubble 1383 Space Telescope images of Asteroid 4 Vesta. Icarus. 128, 83-87. 


\section{Figure captions}

1386

1387

1388

1389

1390

1391

1392

1393

1394

1395

1396

1397

1398

1399

1400

1401

1402

1403

1404

1405

1406

1407

1408

1409

1410

1411

1412

1413

1414

1415

1416

1417

1418

1419

1420

1421

1422

1423

1424

1425

1426

1427

1428

1429
Figure 1. Color shaded relief map of the surface of Vesta. Topography is derived from Dawn Framing Camera data. The coordinate system shows is the "Claudia" system used by the Dawn science team. The map shows the locations of physiographic provinces, major structural centers and impact structures greater than 30 kilometers in diameter. White boxes indicate the locations of type figures (6-22) throughout the paper. Image credit: NASA/JPL/DLR.

Figure 2. Map of Vesta's southern hemisphere based on Framing Camera Rotational Characterization (RC) data. The latitude/longitude coordinate system shown is the "Claudia" system used by the Dawn science team.

Figure 3. Framing Camera RC1 image f2_362695687 with an arbitrary set of longitudes overlaid (the FC team had not settled on a standard latitude/longitude system at this time). Misidentified in the RC-based map as a crater rim (upper arrow) and peak (lower arrow) are the eastern and western rims, respectively, of the craters Marcia, Calpurnia and Minucia.

Figure 4. Geologic map (a) and legend (b) based on Survey orbital data, produced at $1: 1,000,000$. Yellow circles indicate areas identified as having a diogenite (two small circles) or olivine (large circle) signature [Binzel et al., 1997]. Note the locations of "orange"-toned surface material (stippled pattern) mapped as dark mantling material. This and all following figures show coordinates in the "Claudia" system utilized by the Dawn science team.

Figure 5. Geologic map based on HAMO orbital data, as described in text. The map was produced at 1:500,000, using Framing Camera data as the basemap. Vesta is divided latitudinally into four areally extensive units: the Rheasilvia Formation, the Divalia Fossae and Saturnalia Fossae units, and cratered highlands. The blank area to the north represents regions for which there is no HAMO data. a) Simple cylindrical projection; b) North and South polar projections; c) legend.

Figure 6. Mass wasting material (mw). (a) Portion of a fan-shaped deposit in Marcia crater, with dark lobate material ( $\mathrm{dl}$ ) to the east in the crater floor. This deposit is characterized by subtle ridges of material radiating downslope. Note the individual boulders visible in the deposit. The center of this LAMO mosaic is at latitude $9.7^{\circ} \mathrm{N}$., longitude $186.0^{\circ} \mathrm{E}$ in the Claudia coordinate system. North is up. (b) Slump deposit south of Matronalia Rupes that lies between Rheasilvia smooth terrain (Rs) and the Rheasilvia ridge-and-groove terrain (Rrg). Here, mw is comprised of subparallel blocks sliding downslope. The center of this LAMO mosaic is at latitude $54.5^{\circ} \mathrm{S}$, longitude $91.9^{\circ}$ E. LAMO mosaic. North is up. Image credit: NASA/JPL/DLR.

Figure 7. Bright lobate material (bl). This type area lies south of Aricia Tholus. The bright lobate material is the smooth tongue of material draping the western rim of the 
1430

1431

1432

1433

1434

1435

1436

1437

1438

1439

1440

1441

1442

1443

1444

1445

1446

1447

1448

1449

1450

1451

1452

1453

1454

1455

1456

1457

1458

1459

1460

1461

1462

1463

1464

1465

1466

1467

1468

1469

1470

1471

1472

1473

1474

crater. The center of this HAMO mosaic is at latitude $1.6^{\circ} \mathrm{S}$., longitude $163.6^{\circ} \mathrm{E}$. North is up. Image credit: NASA/JPL/DLR.

Figure 8. Dark lobate material (dl). This type area lies in Octavia crater, within a patch of dark crater (dc) material. Here, dl appears fine-textured, with rolling topography. The center of this LAMO mosaic is at latitude $3.4^{\circ} \mathrm{S}$., longitude $147.6^{\circ} \mathrm{E}$. North is up. Image credit: NASA/JPL/DLR.

Figure 9. Smooth material (s). This type area is in Marcia crater, lying on a southern bench between the rim and a mass wasting ( $\mathrm{mw}$ ) deposit downslope to the north. Note the difference in texture between smooth material and the more hummocky mw material. The center of this LAMO mosaic is at latitude $3.6^{\circ} \mathrm{N}$, longitude $187^{\circ} \mathrm{E}$. North is up. Image credit: NASA/JPL/DLR.

Figure 10. Tholus material ( $\mathrm{t}$ ). This type area is Aricia Tholus, The tholus is coneshaped, with a cratered surface; there is no indication of flows or other volcanic products associated with this structure. The center of this LAMO mosaic is at latitude $10^{\circ} \mathrm{N}$, longitude $160^{\circ} \mathrm{E}$. North is up. Image credit: NASA/JPL/DLR.

Figure 11. Cratered highlands material (ch). The type area is northeast of Numisia crater. Note the abundance of overlapping craters of various sizes and states of degradation; many have subcircular, rather than circular perimeters. The center of this LAMO mosaic is at latitude $5.0^{\circ} \mathrm{N}$., longitude $260.5^{\circ} \mathrm{E}$. North is up. Image credit: NASA/JPL/DLR.

Figure 12. Cratered plains material (cp). This type area is southwest of Drusilla crater. While many impact structures are present, the craters are smaller and fewer. The center of this LAMO mosaic is at latitude $23.5^{\circ} \mathrm{S}$., longitude $239.5^{\circ} \mathrm{E}$. North is up. Image credit: NASA/JPL/DLR.

Figure 13. Bright crater ray material (bcr). This type area is at Canuleia crater, which formed in Rheasilvia ridge-and-groove ( $\mathrm{Rrg}$ ) terrain. Note the light-toned streaks radiating from the crater are not uniform; this is common for bcr material. The center of this LAMO mosaic is at latitude $33.8^{\circ} \mathrm{S}$., longitude $294.5^{\circ} \mathrm{E}$. North is up. Image credit: NASA/JPL/DLR.

Figure 14. Dark crater ray material (dcr). This type area is at Arruntia crater, within the Saturnalia Fossae unit (Sf). Discontinuous dark-toned streaks radiate from the crater. Here, the low sun angle reveals the rough, mesh-like texture of Sf, which is mantled by dcr. The center of this LAMO mosaic is at latitude $39.0^{\circ} \mathrm{N}$., longitude $75.2^{\circ} \mathrm{E}$. North is up. Image credit: NASA/JPL/DLR.

Figure 15. Bright crater material (bc). This type area is southwest of Lucaria Tholus, at a junction between the Rheasilvia smooth terrain (Rs) and the Divalia Fossae unit (Df). The texture is similar to that of the Rs terrain, but is slightly rougher and lighter-toned. 
1475 The center of this LAMO mosaic is at latitude $22.7^{\circ} \mathrm{S}$., longitude $85.2^{\circ} \mathrm{E}$. North is up. 1476 Image credit: NASA/JPL/DLR.

1477

1478

1479

1480

1481

1482

1483

1484

1485

1486

1487

1488

1489

1490

1491

1492

1493

1494

1495

1496

1497

1498

1499

1500

1501

1502

1503

1504

1505

1506

1507

1508

1509

1510

1511

1512

1513

1514

1515

1516

1517

1518

1519

1520
Figure 16. Dark crater material (dc). This type area is south of Laelia crater, which formed in Rheasilvia smooth terrain (Rs). Note the similarity in texture to bc, but darker-toned than the surrounding Rs unit. The center of this LAMO mosaic is at latitude $51.3^{\circ}$ S., longitude $140.7^{\circ}$ E. North is up. Image credit: NASA/JPL/DLR.

Figure 17. Undifferentiated ejecta material (uc). Here uc material lies near Gegania crater, in the Divalia Fossae unit (Df). The texture is smoother than the surrounding unit but otherwise morphologically similar. The center of this LAMO mosaic is at latitude $3.1^{\circ} \mathrm{N}$., longitude $63.1^{\circ} \mathrm{E}$. North is up. Image credit: NASA/JPL/DLR.

Figure 18. Saturnalia Fossae material (Sf). This type area lies west of Scantia crater, along a group of ridges. Note the high crater density and the surface texture, which appears more rough than the Divalia Fossae unit, but this may simply be a question of the illumination being more conducive to revealing roughness. The center of this LAMO mosaic is at latitude $33.1^{\circ} \mathrm{N}$., longitude $236.3^{\circ} \mathrm{E}$. North is up. Image credit: NASA/JPL/DLR.

Figure 19. Divalia Fossae material (Df). This type area is south of Gegania crater, just south of the cratered highlands (ch) and intersected by a deposit of crater ejecta, undifferentiated (uc). Here, Df displays subparallel ridges, less heavily cratered than the ch deposit to the north. The center of this LAMO mosaic is at latitude $9.6^{\circ} \mathrm{S}$, longitude $61.4^{\circ}$ E. North is up. Image credit: NASA/JPL/DLR.

Figure 20. Rheasilvia smooth material (Rs). This type area lies west of Pinaria crater south of the Divalia Fossae unit. Note the smooth topography, flatter and less cratered than Df. This LAMO mosaic is centered at latitude $30^{\circ} \mathrm{S}$., longitude $354^{\circ} \mathrm{E}$. North is up. Image credit: NASA/JPL/DLR.

Figure 21. Rheasilvia ridge-and-groove material (Rrg). This type area lies northwest of Severina crater. Here, radial grooves and ridges curve between deposits of uc (south) and $\mathrm{mw}$ (north). The center of this south polar projection LAMO mosaic is at latitude $67^{\circ} \mathrm{S}$., longitude $88.7^{\circ} \mathrm{E}$. Arrow indicates direction of true north. Image credit: NASA/JPL/DLR.

Figure 22. Rheasilvia mound material $(\mathrm{Rm})$. This type area is west of Severina crater. The unit is hummocky, with mw material commonly lying within low regions. In this case, a tongue of mw occurs to the east between a crater and a rugged ridge. The center of this south polar projection LAMO mosaic lies at latitude $74.7^{\circ} \mathrm{S}$., longitude $279^{\circ} \mathrm{E}$. Arrow indicates direction of true north. Image credit: NASA/JPL/DLR.

Figure 23. Crater statistics data based on the method of Marchi et al. [2012a]. The left panel shown the model production function best fit of the Rrg unit which results in an age of 1.2+-0.2 Ga. The right panel shows the crater SFDs for Sf, ch, and Df units. The 
1521 age for Rrg is slightly higher than a previous estimate of $\sim 1.0+/-0.2$ Ga based on 1522 Rheasilvia whole floor counts [from Marchi et al., 2012a]. The difference is due to a 1523 better definition of the counting area: the average crater density on Rrg unit is slightly 1524 higher than the average over the whole Rheasilvia floor. This figure shows that the MPF 1525 reproduces relatively well the shape of the carter SFD [see Marchi et al. [this volume] 1526 for more details). The apparent lack of crater $<3.5 \mathrm{~km}$ is ascribed to incomplete crater 1527 identification.

1528

1529

1530 1531

1532

1533

Figure 24. Crater size-frequency distribution functions for the major geologic units described. (a) ch; (b) Sf; (c) Df; (d) cp; (e) Rs; (f) Rrg; (g) Rm; (h) mw; (i) t; (j) bl; (k) dl.

1534

1535

Figure 25. Column showing the correlation of material units as a function of time. Age decreases from the bottom to the top of the figure. 


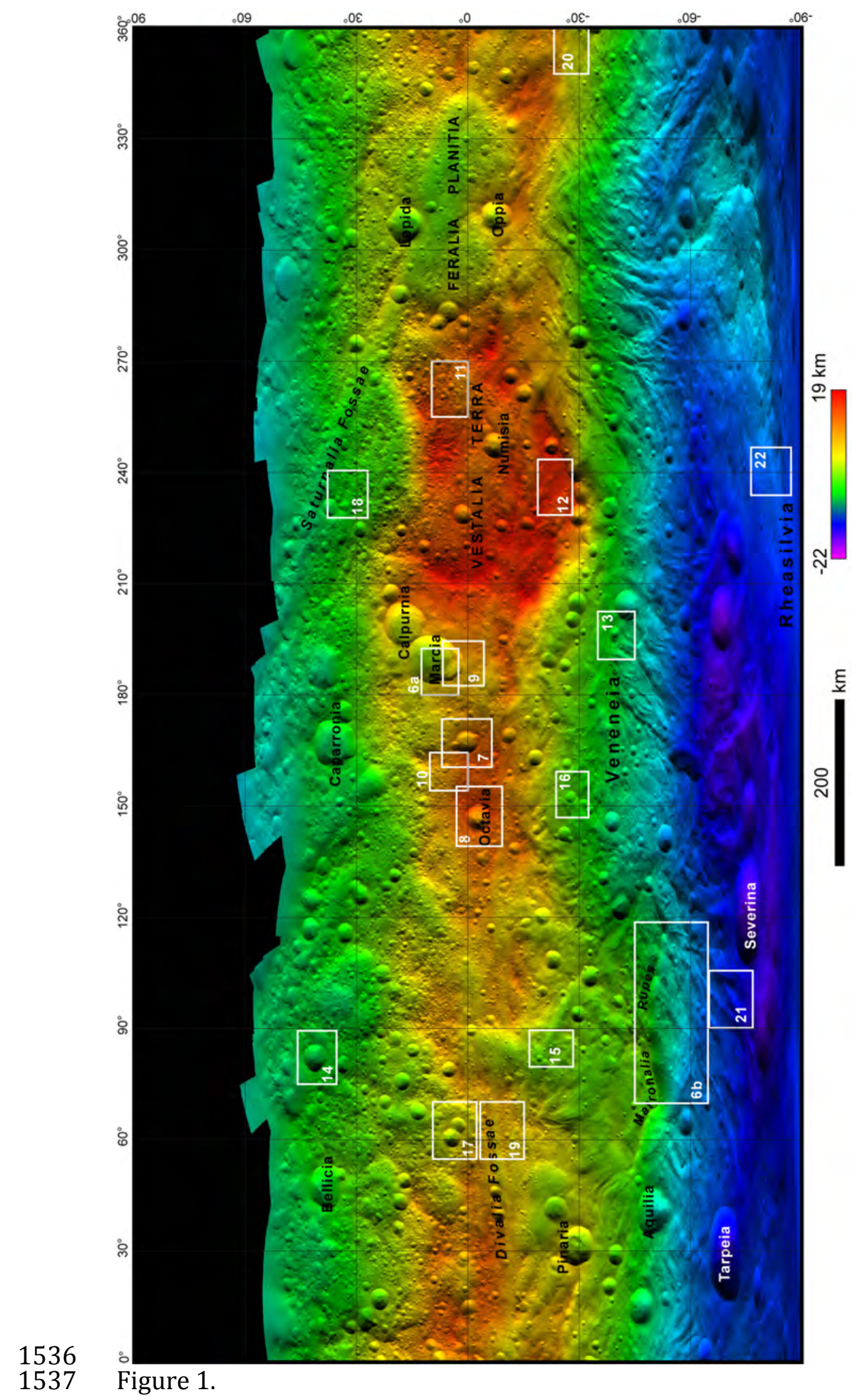


Geologic Sketch Map of Vesta South Pole, RC1, RC2 \& OpNav 16 \& 17, 1-17 July 2011

H1 (Hill 1): Positive
relief mound at SP. relief mound at SP. of SP crater

D1 (Dark Terrain 1): Low albedo material near crater c2, possibly low-lying. Corresponds in $\mathrm{FC}$ color images. in $\mathrm{FC}$ color images.
Interp.: Distinct composintions lated to crater ejecta.

D2: Low albedo unit, likely low-lying, bright red in FC

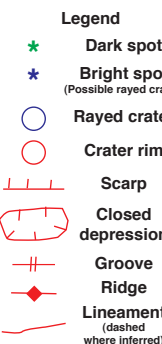
acterized by a prominent scarp. where interred nterp.: Distinct compositional unit; possibly related to crater ejecta.

BT1: High albedo unit, rough in texture; possible overlap with orange terrain. No obvious corresponding terrain in $\mathrm{FC}$ color.

Interp.: Possibly primary crustal material, may or may not be associated with crater ejecta. Another possibility is that this terrain is not a discrete unit, pest available

BT2 (Bright Terrain 2): High albedo unit, rough texture, likely higher than D2, pink in color (BT1). interp.: Primary crustal material, may or may not be associated w/crater ejecta.

GHT: Medium albedo hummocky surface distinguished by sub-radial trending grooves; light gray/red in color images.

Interp.: Material disrupted by SP impact; SP floor.

RT1: Rough, cratered terrain of medium albedo and gray/pink FC color.

atively generic term for undifferntiated crustal terrain.

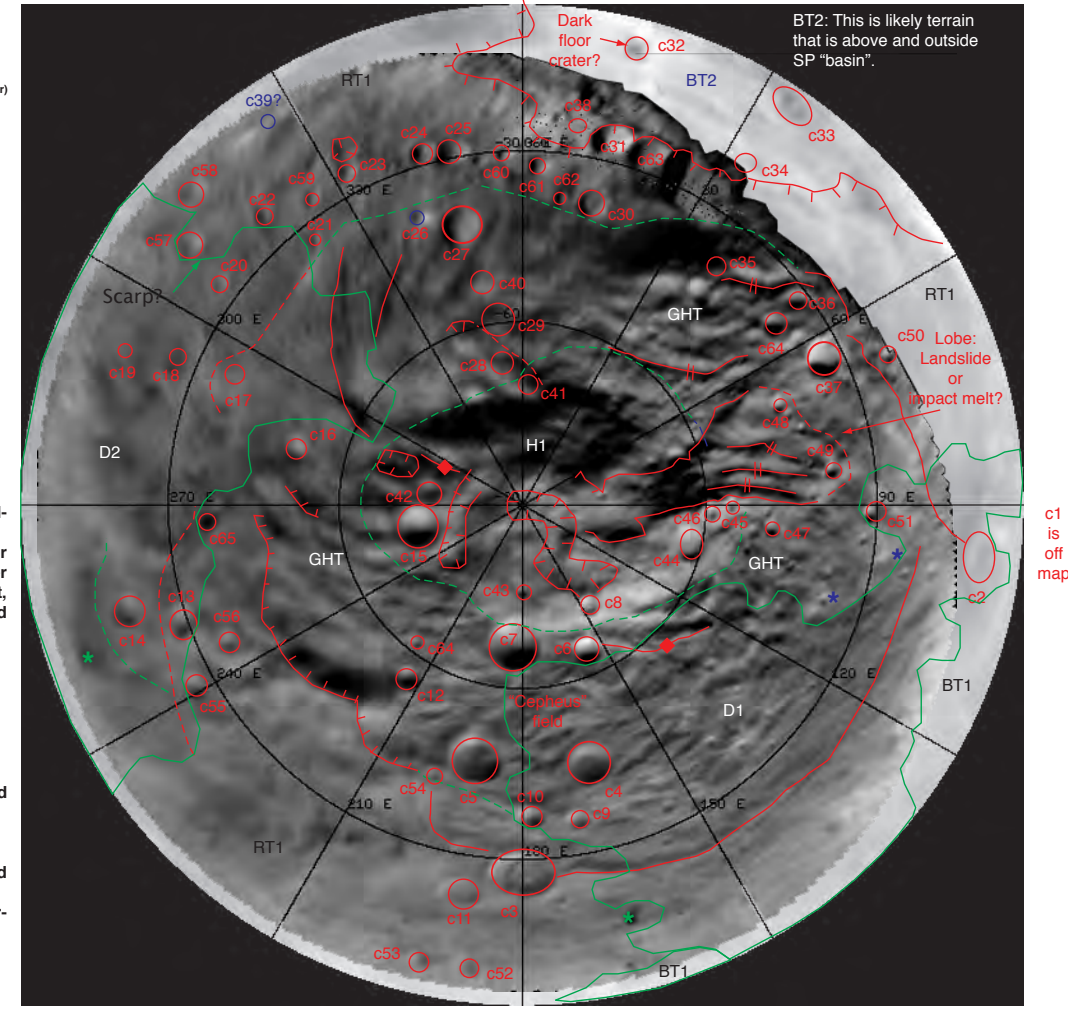

Mapping by R. Aileen Yingst (PSI) \& David A. Williams (ASU). Cartographic processing by Thomas Roatsch (DLR). 
1541

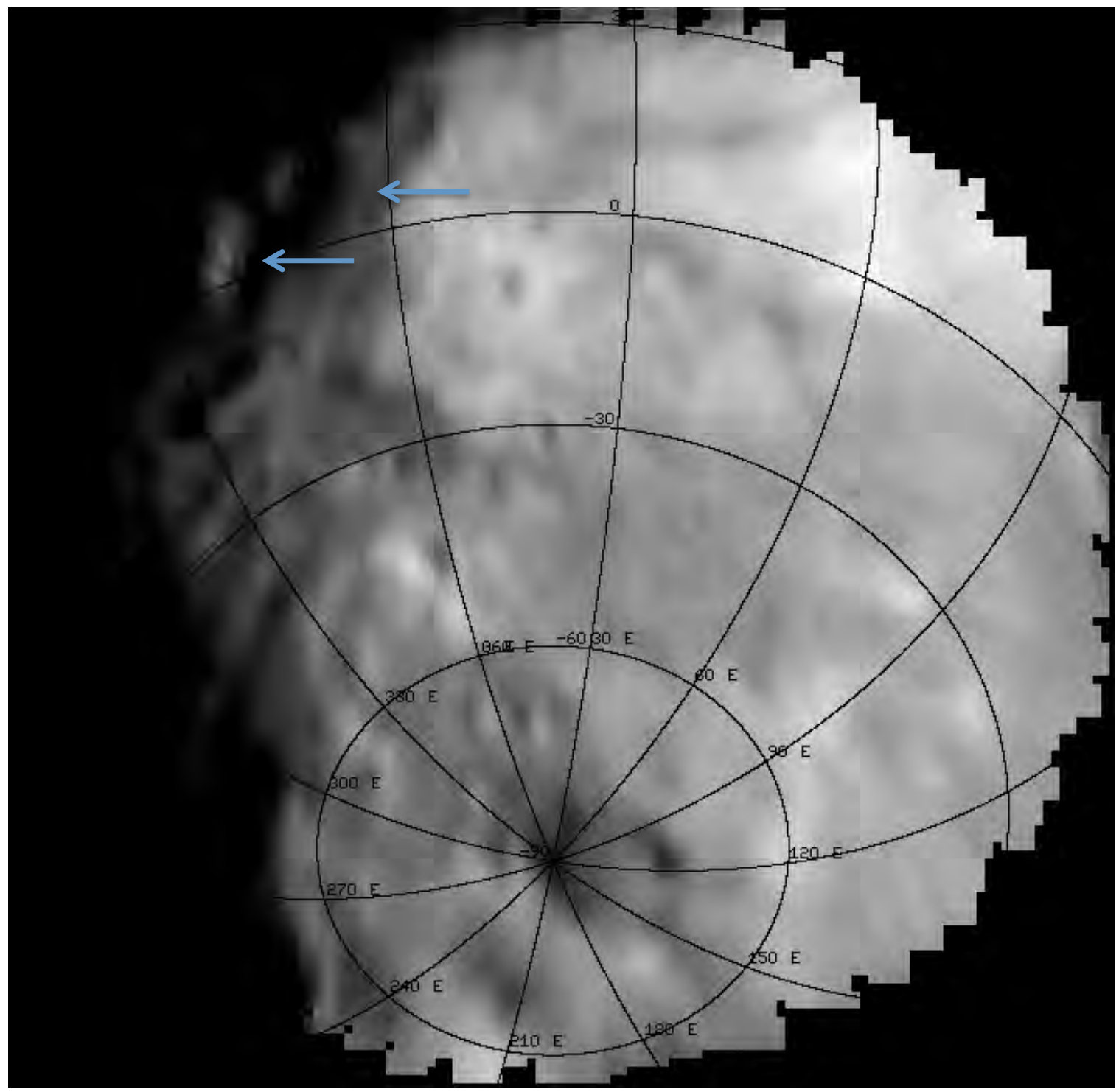

1543

1544

1545

Figure 3.

1546 


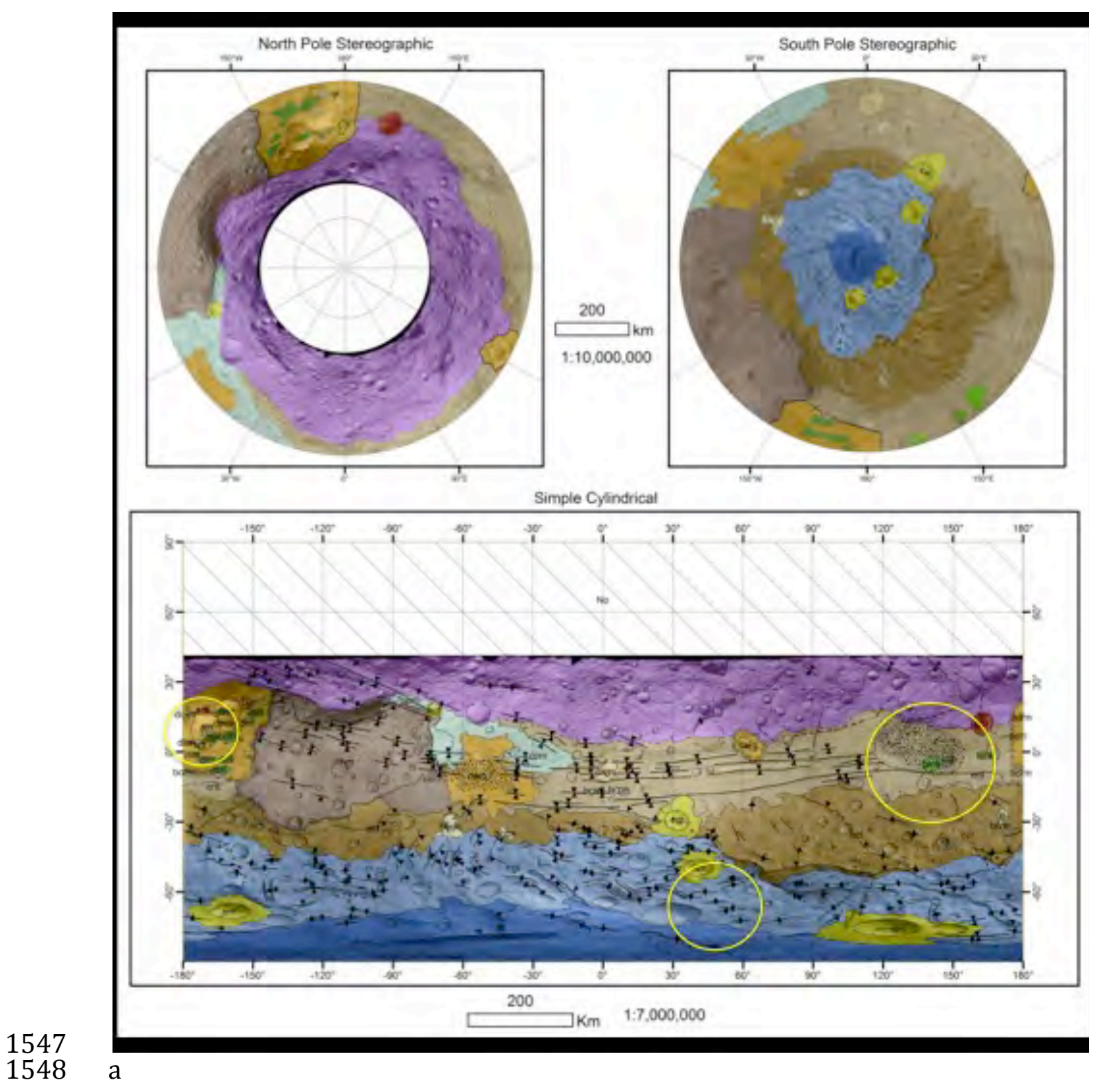




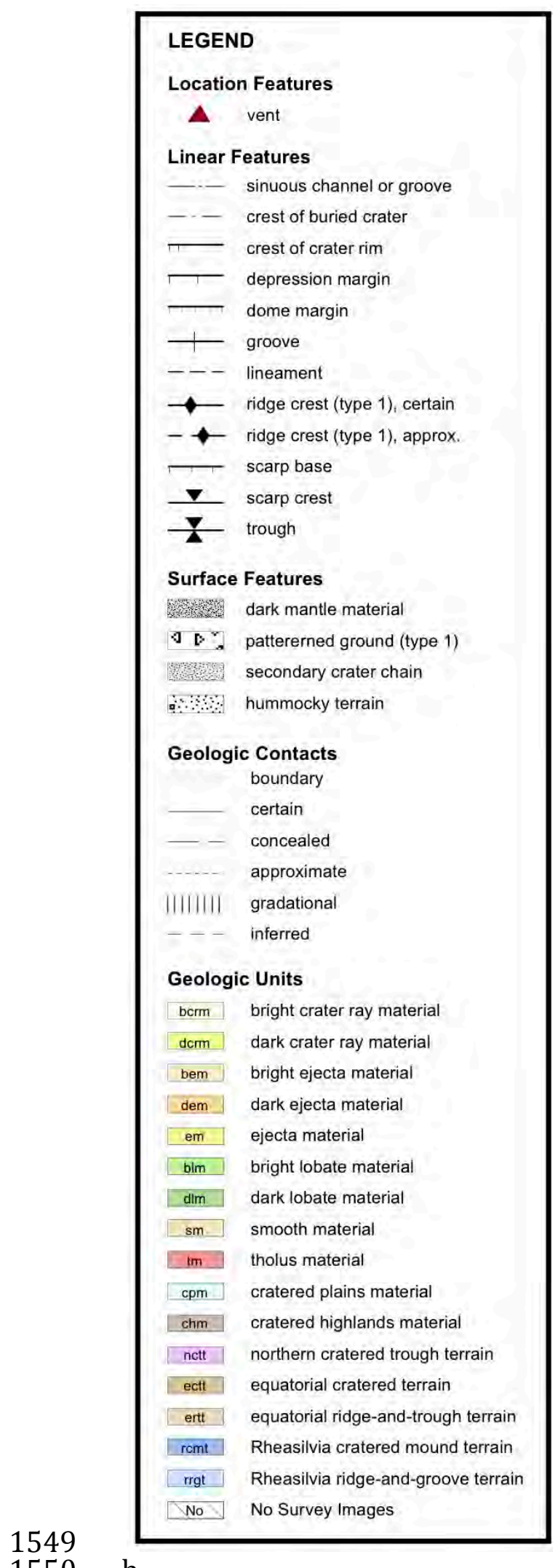

1550 b

1551 Figure 4. 


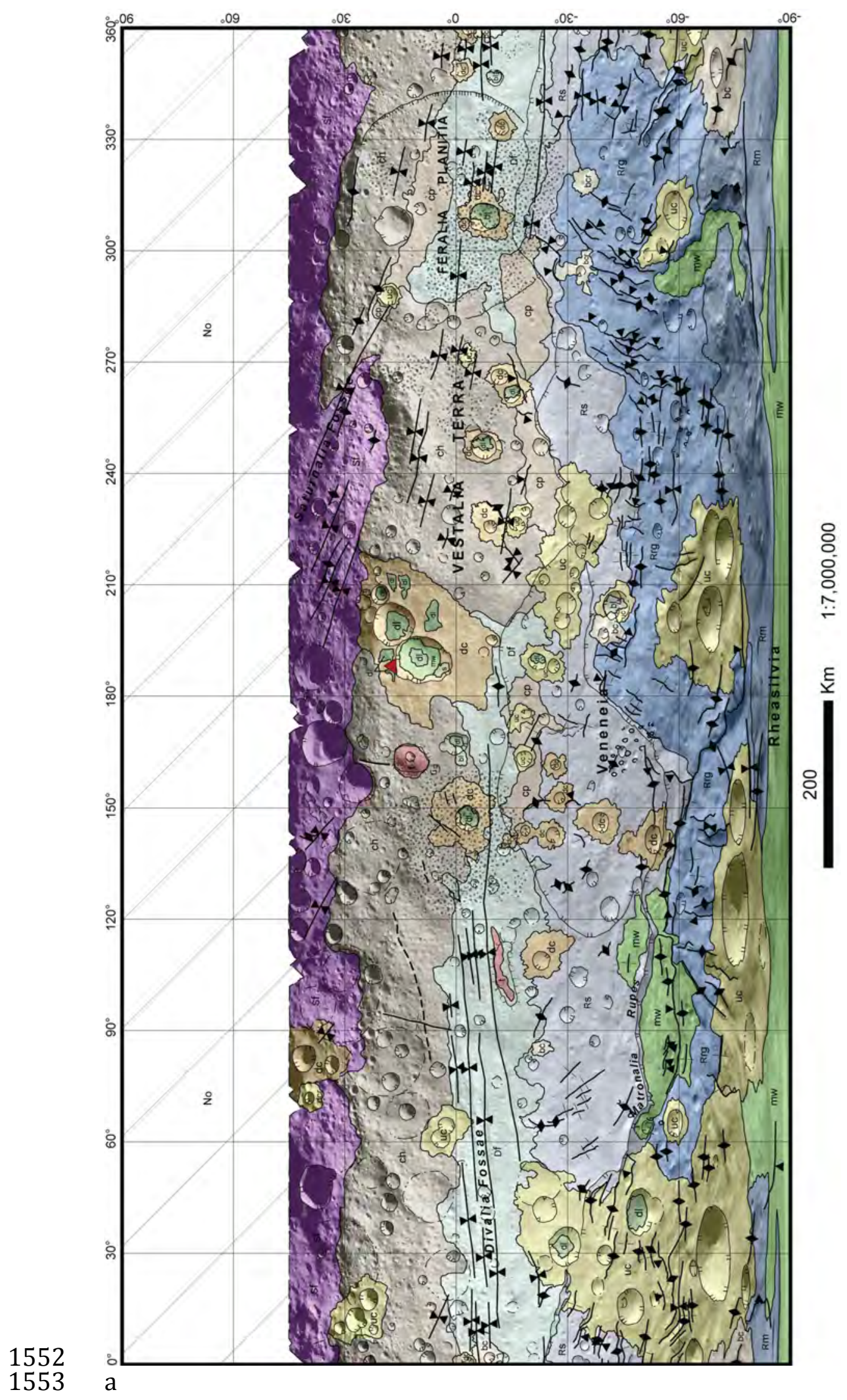


1554

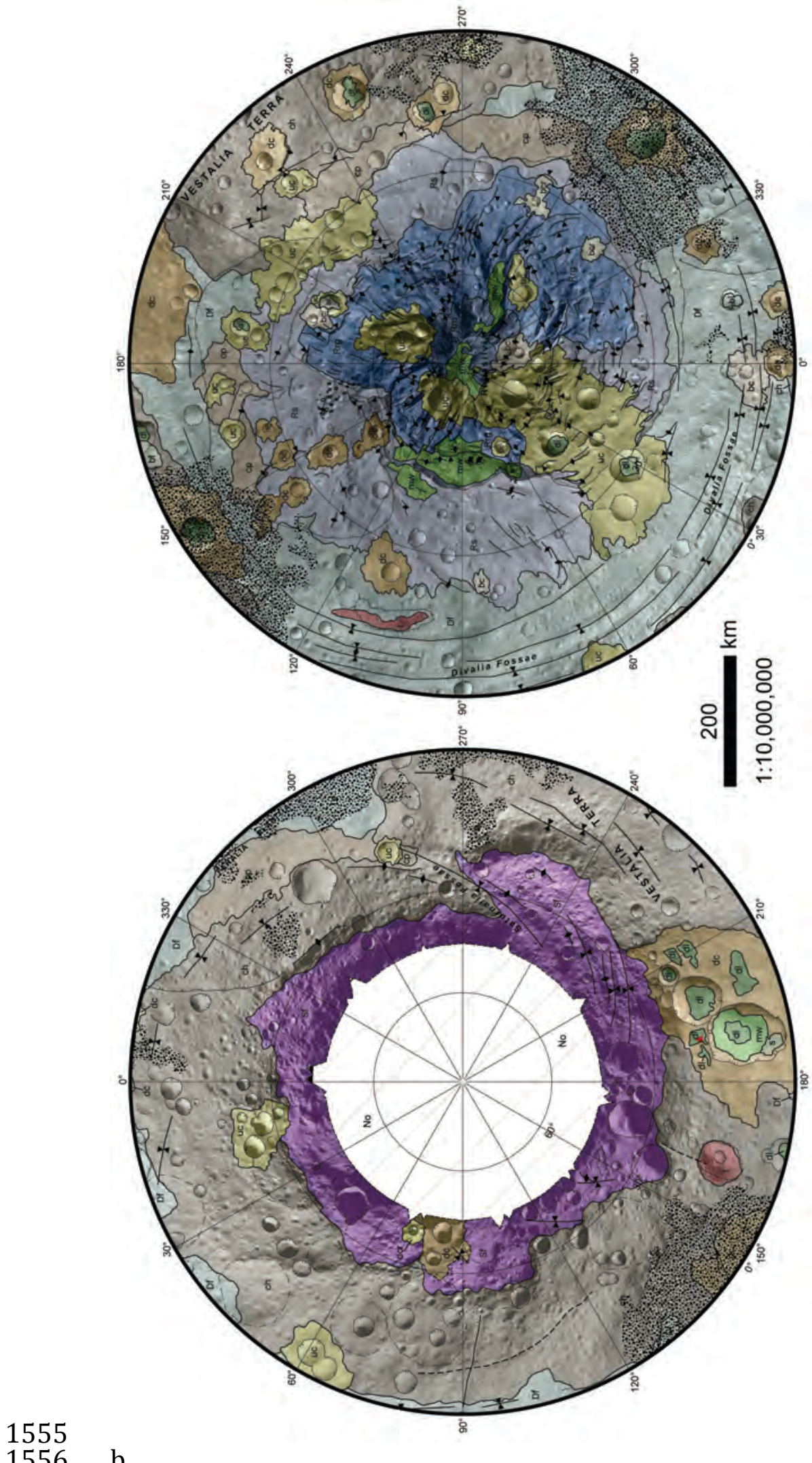




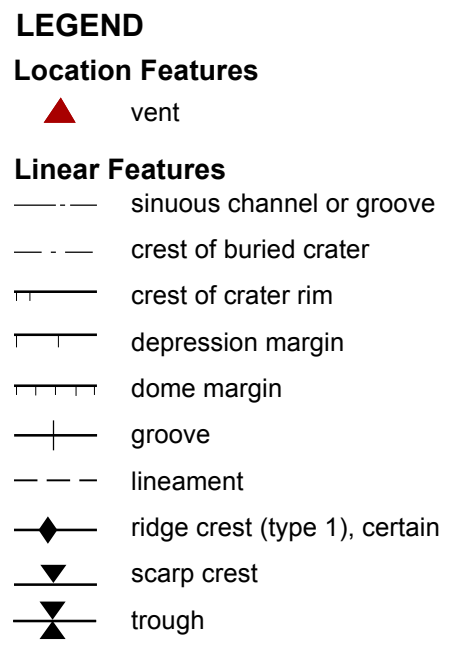


1561

1562

1563

1564

1565

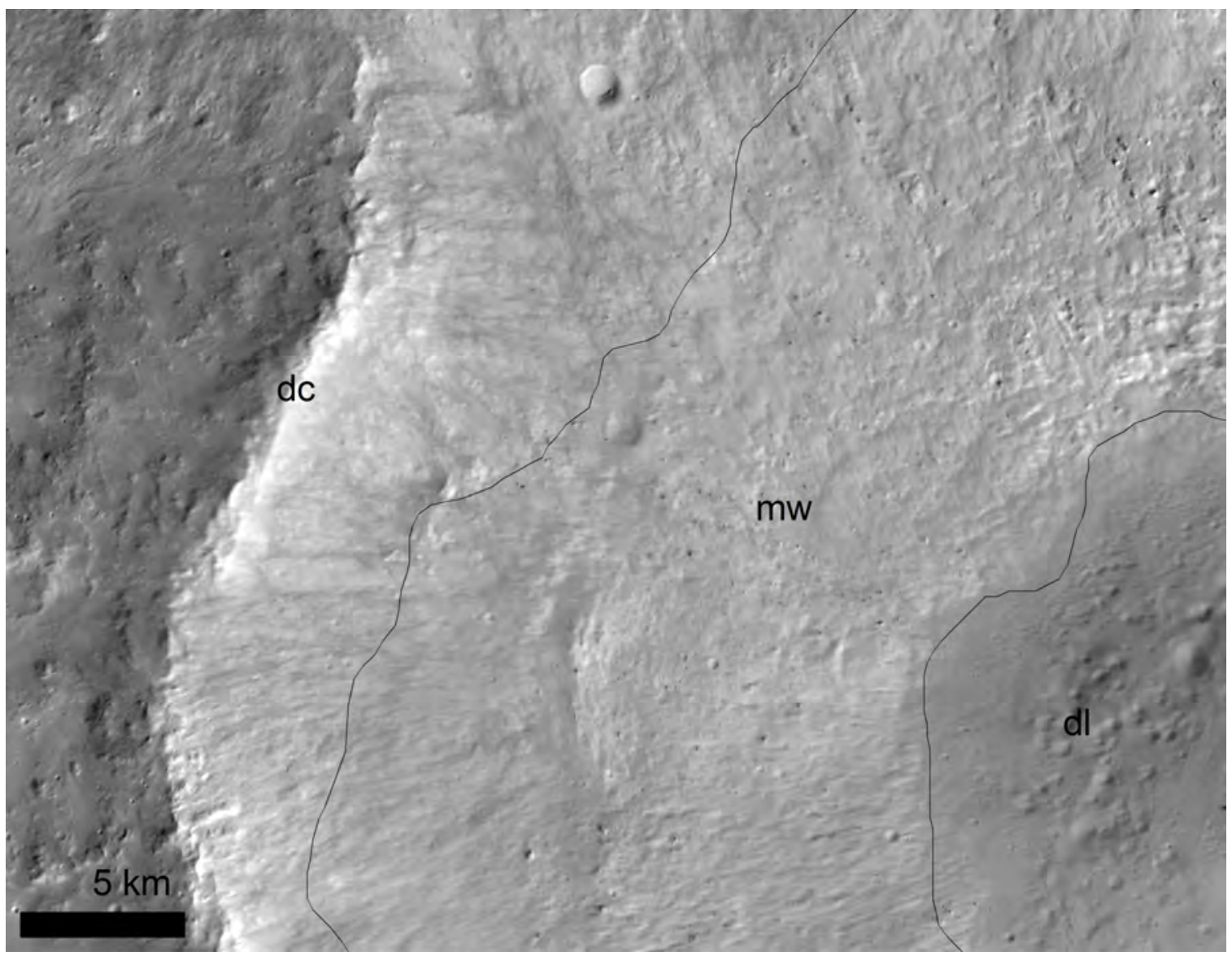

1566 a 


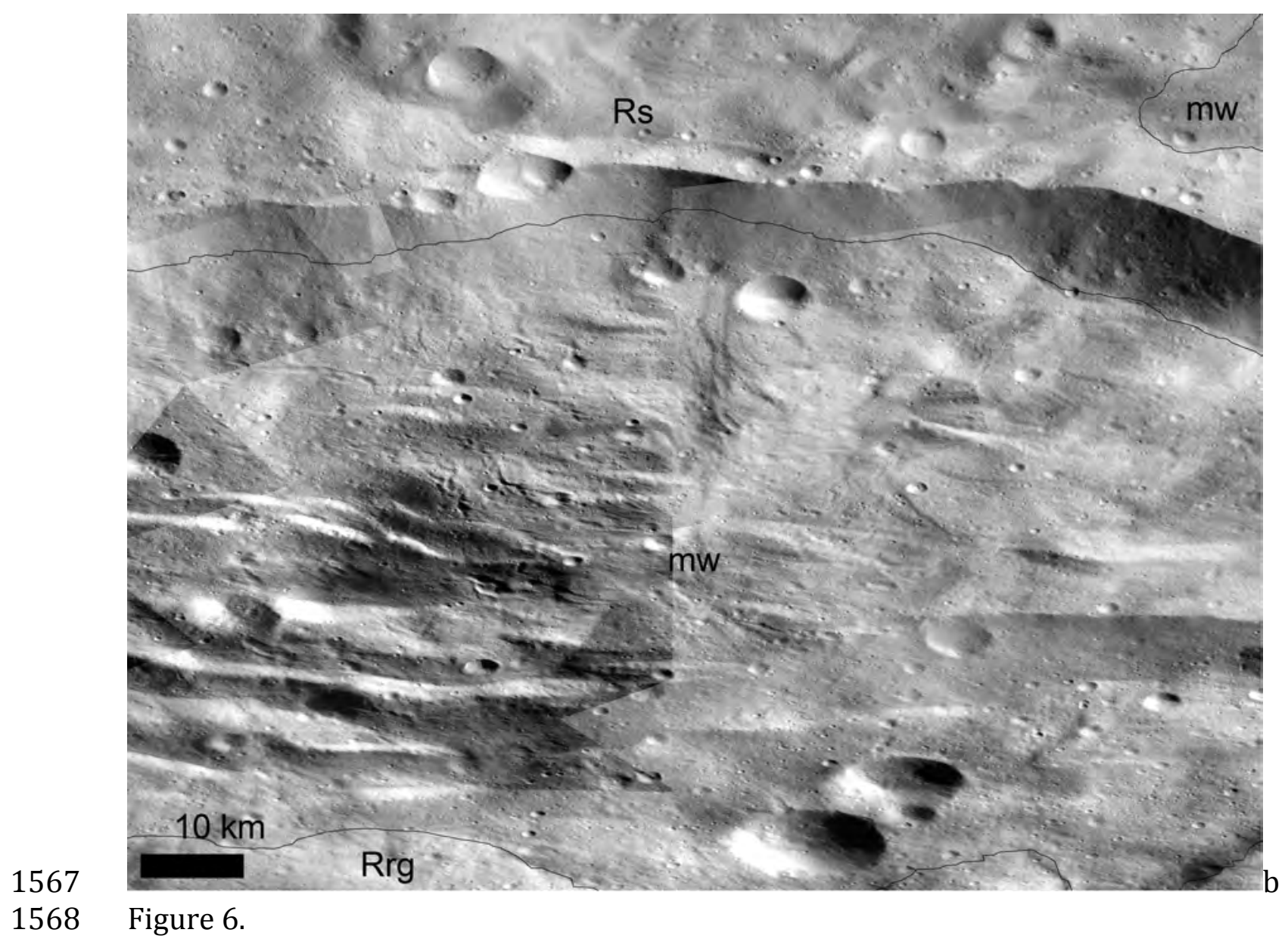




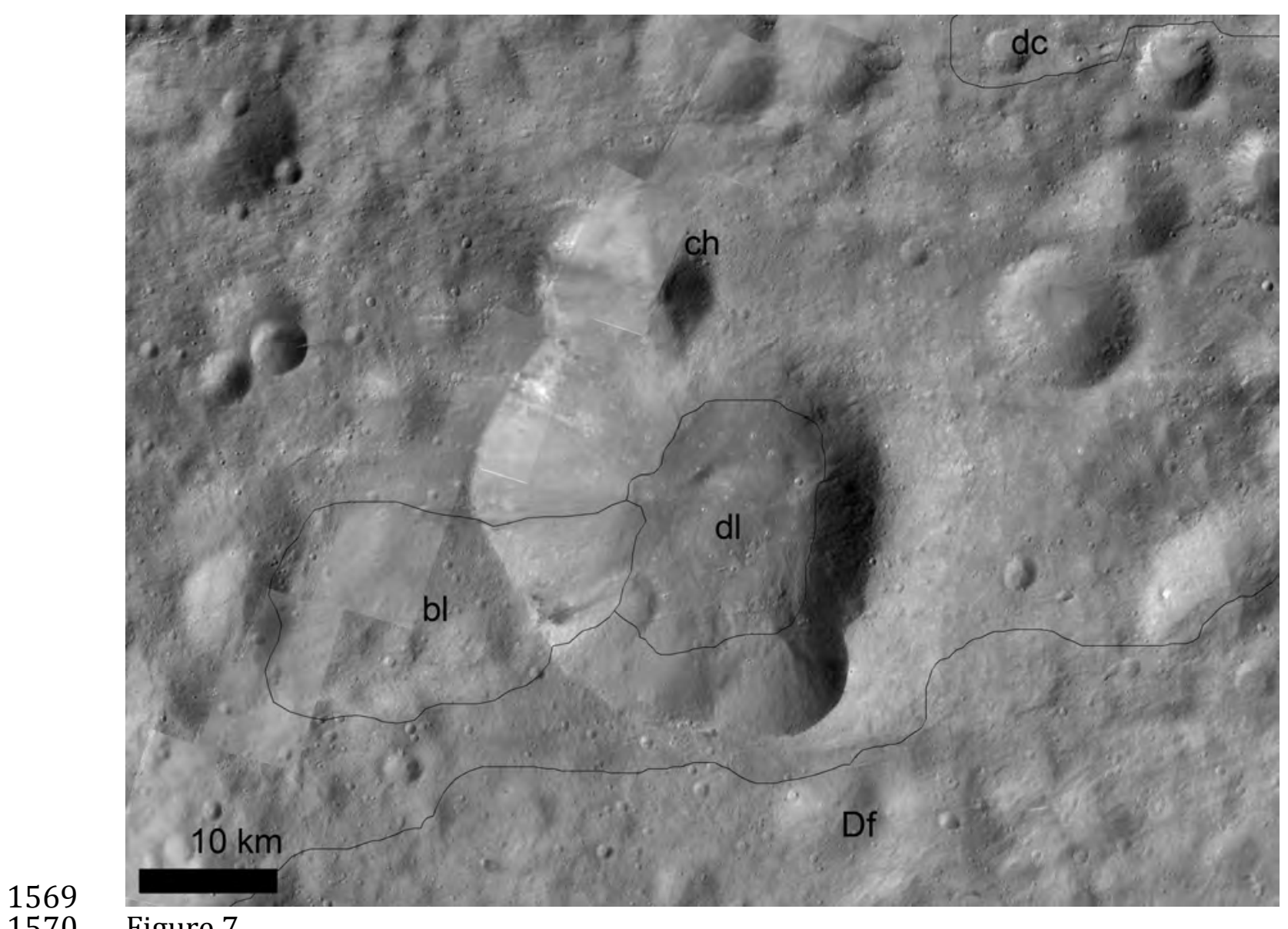

1570 Figure 7.

1571 


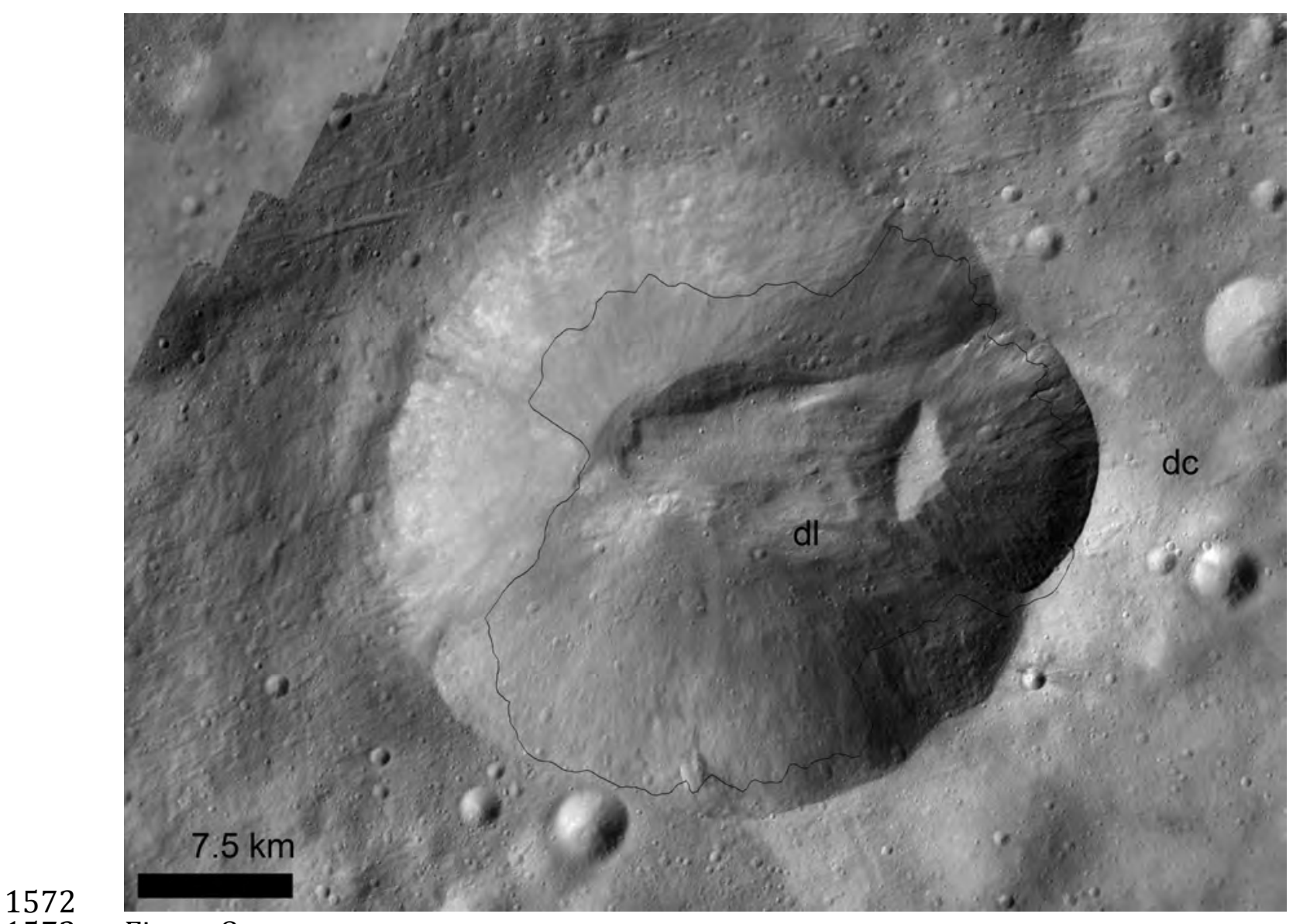

1573 Figure 8. 


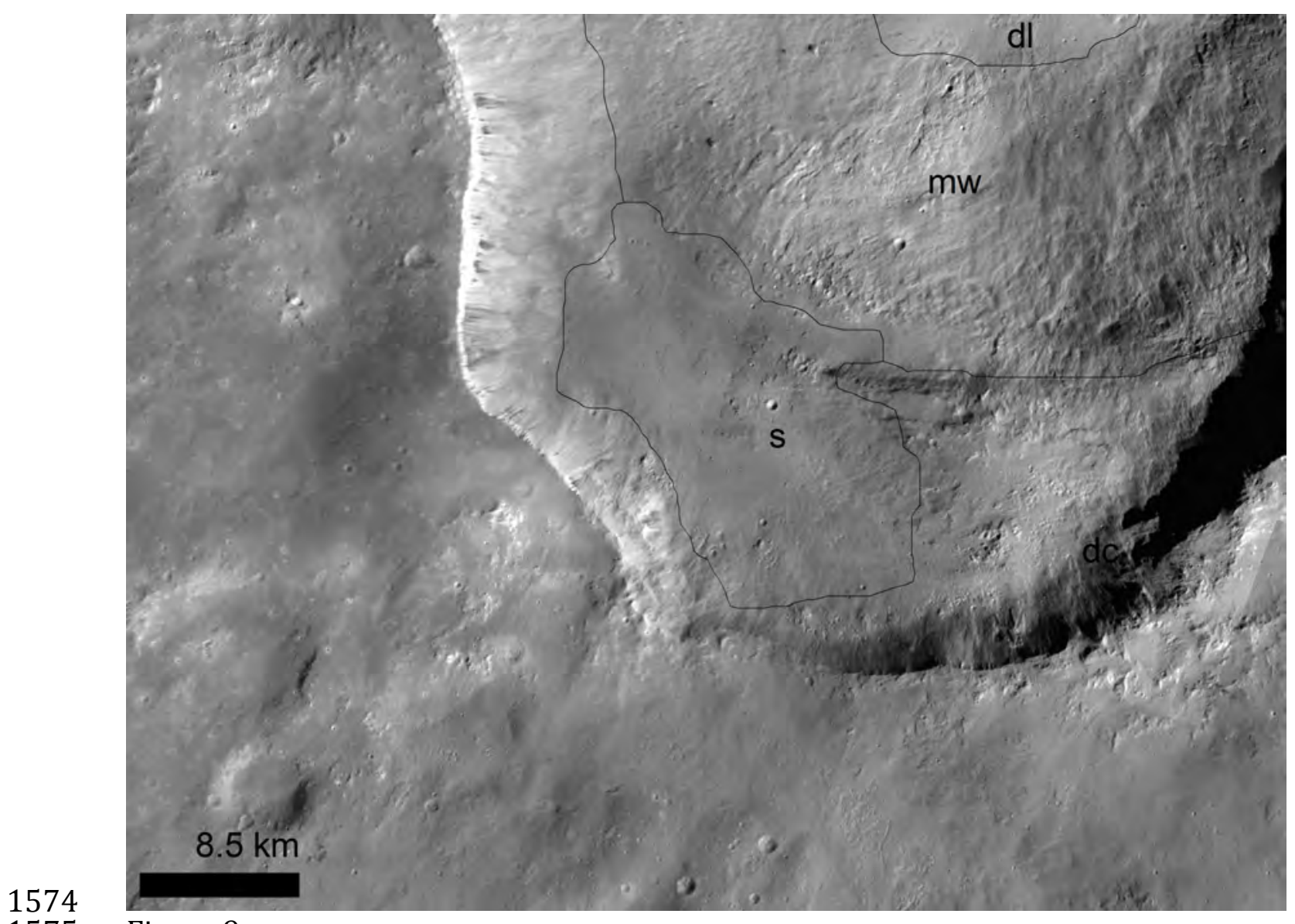

1575 Figure 9. 


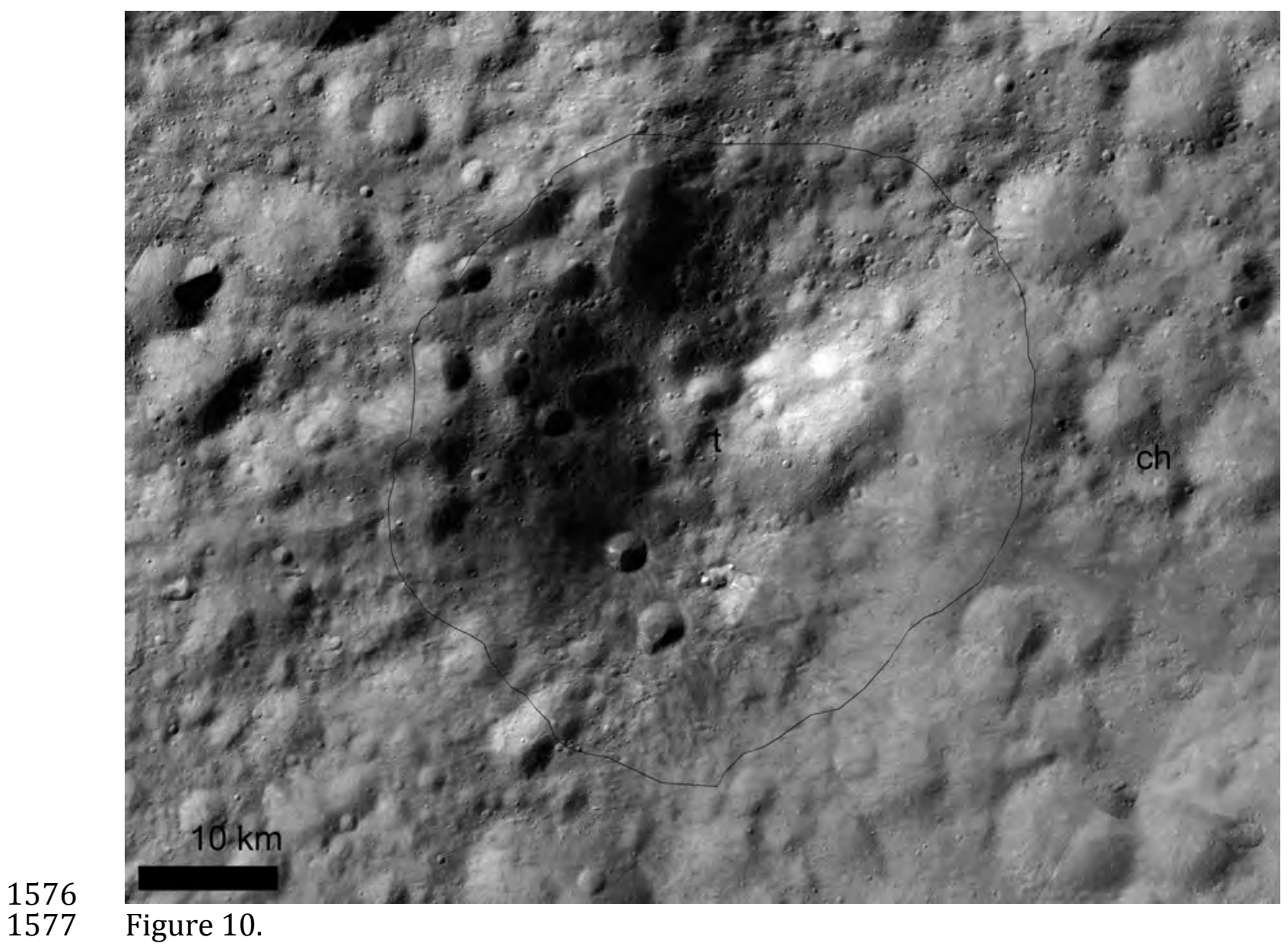




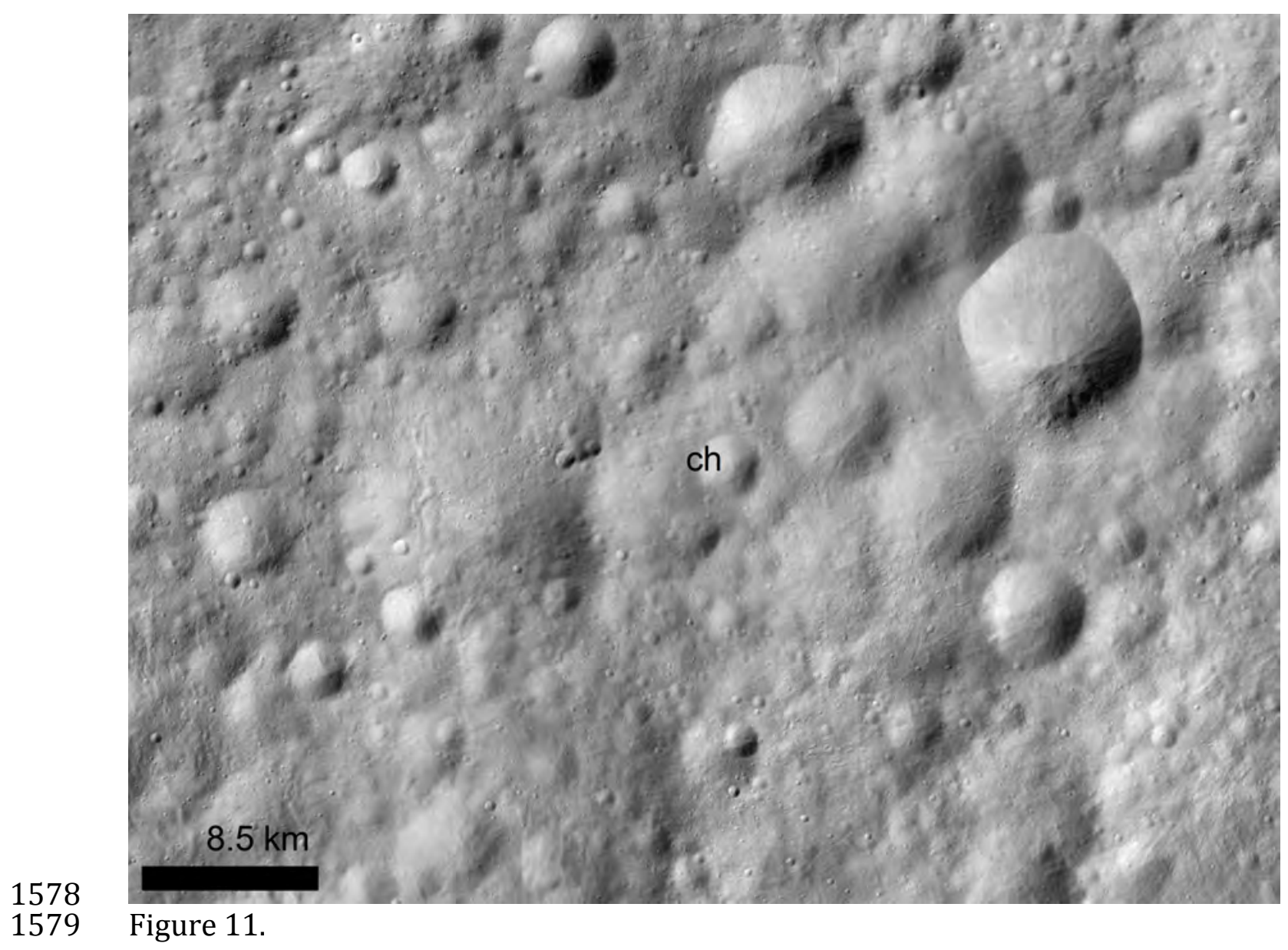




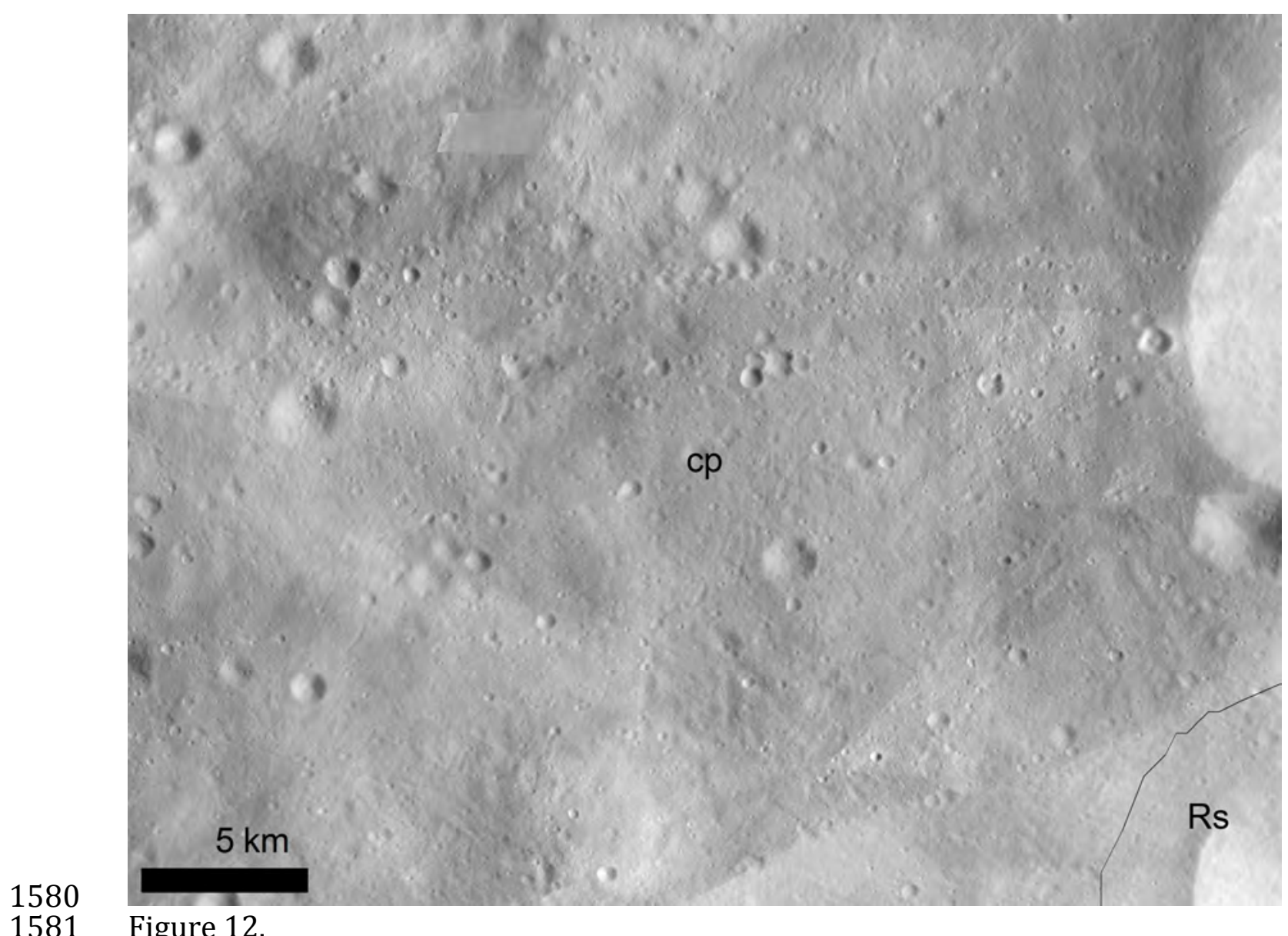

1581 Figure 12. 


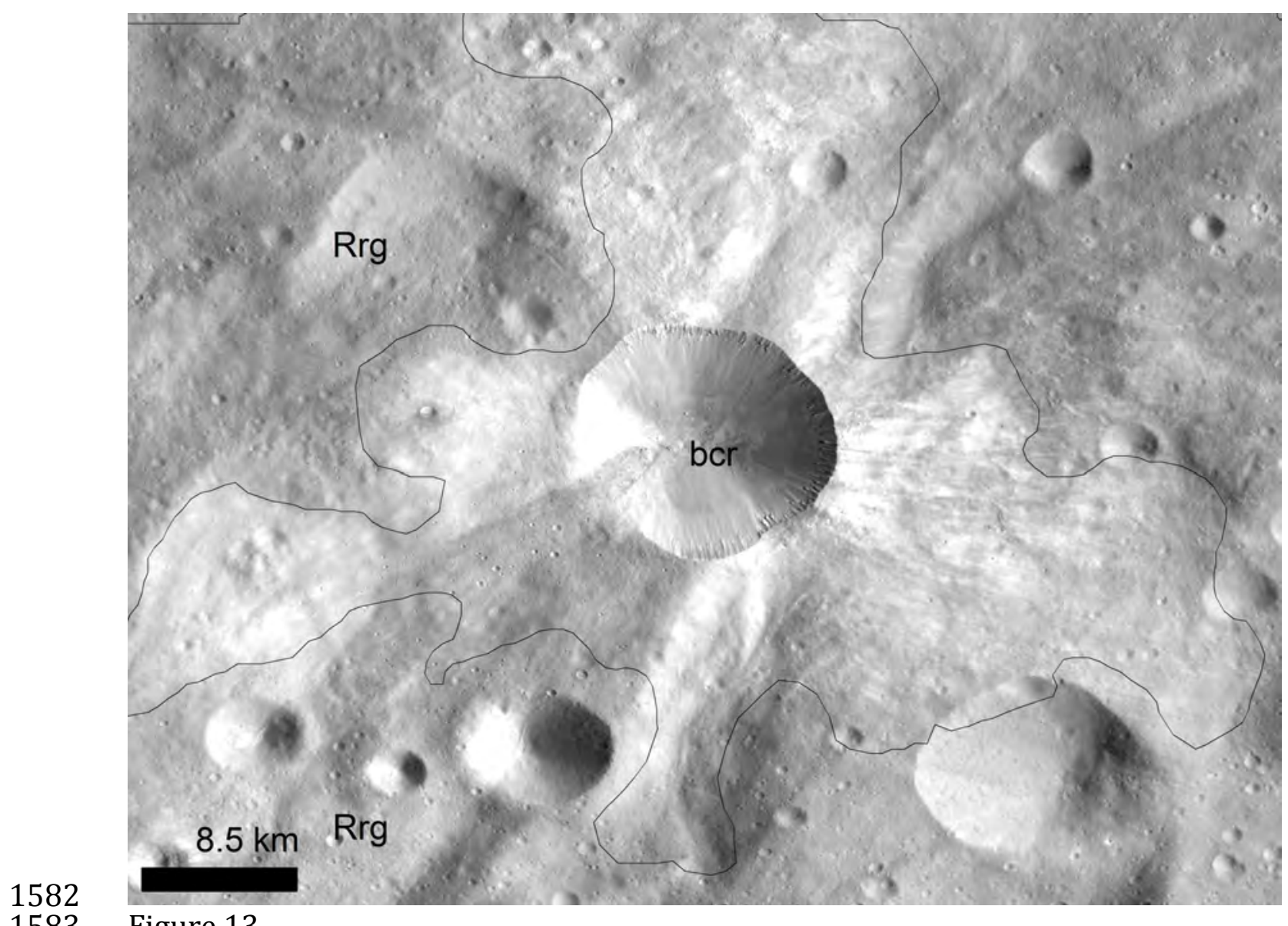

1583 Figure 13. 


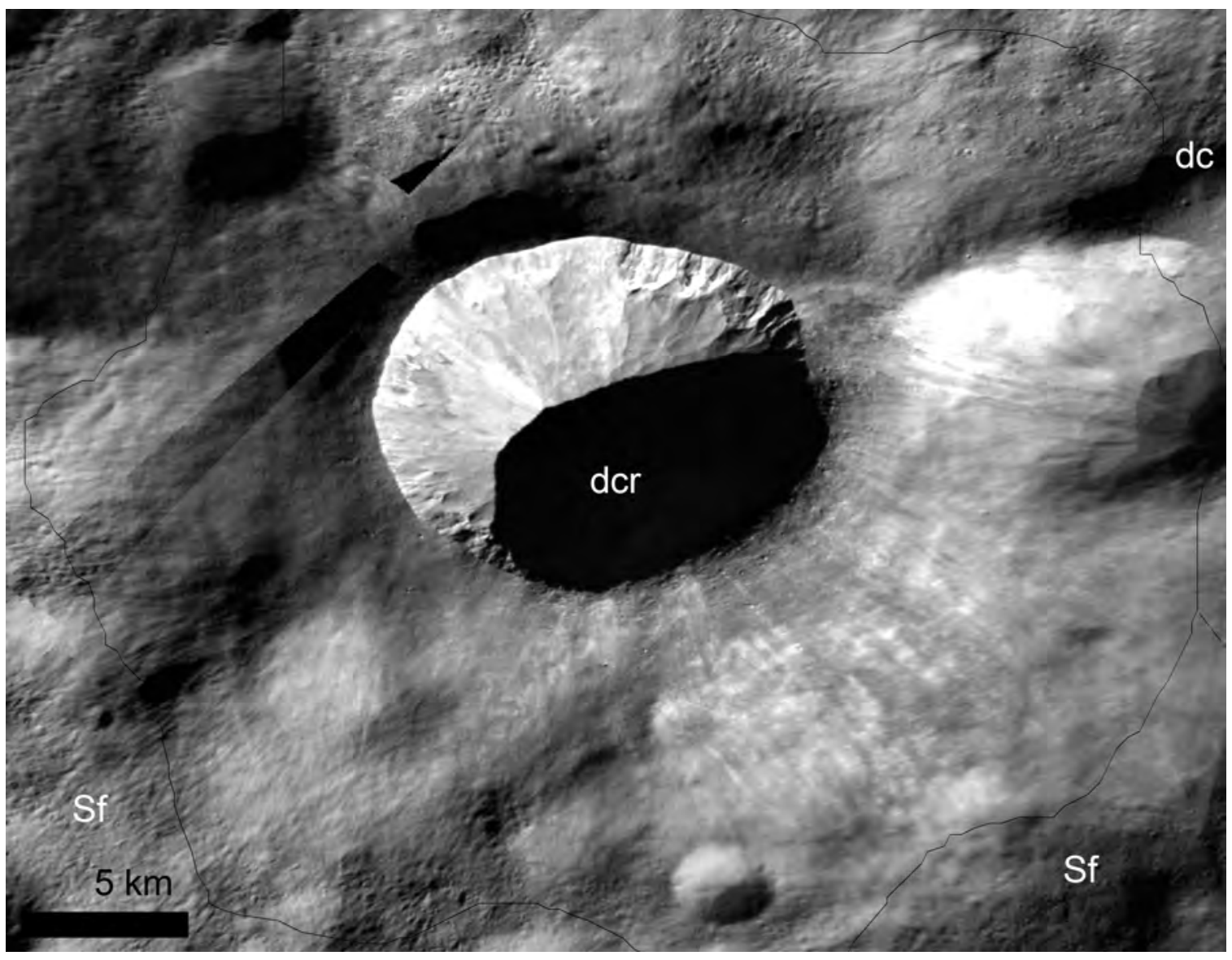

1584 Figure 14. 


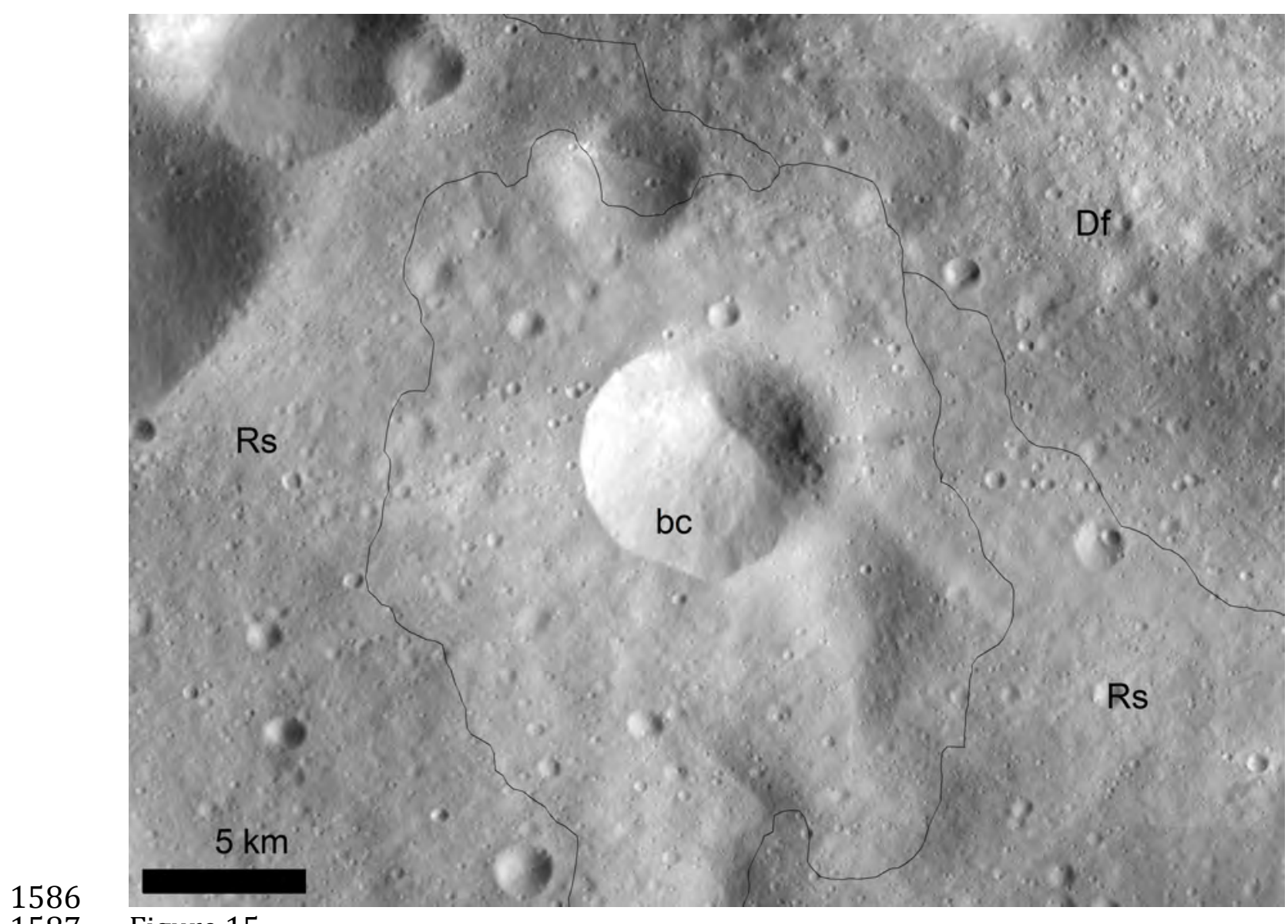

1587 Figure 15. 


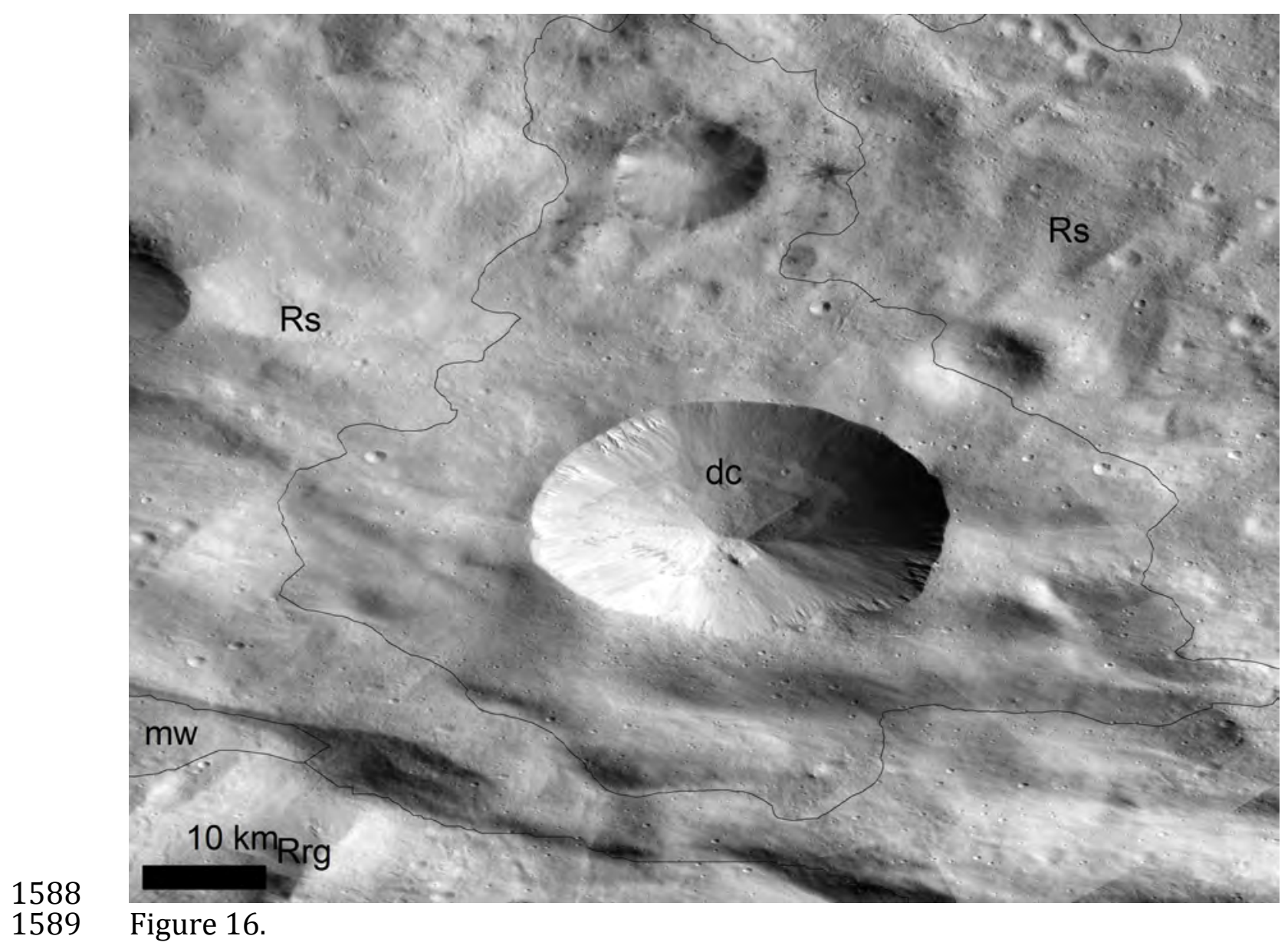




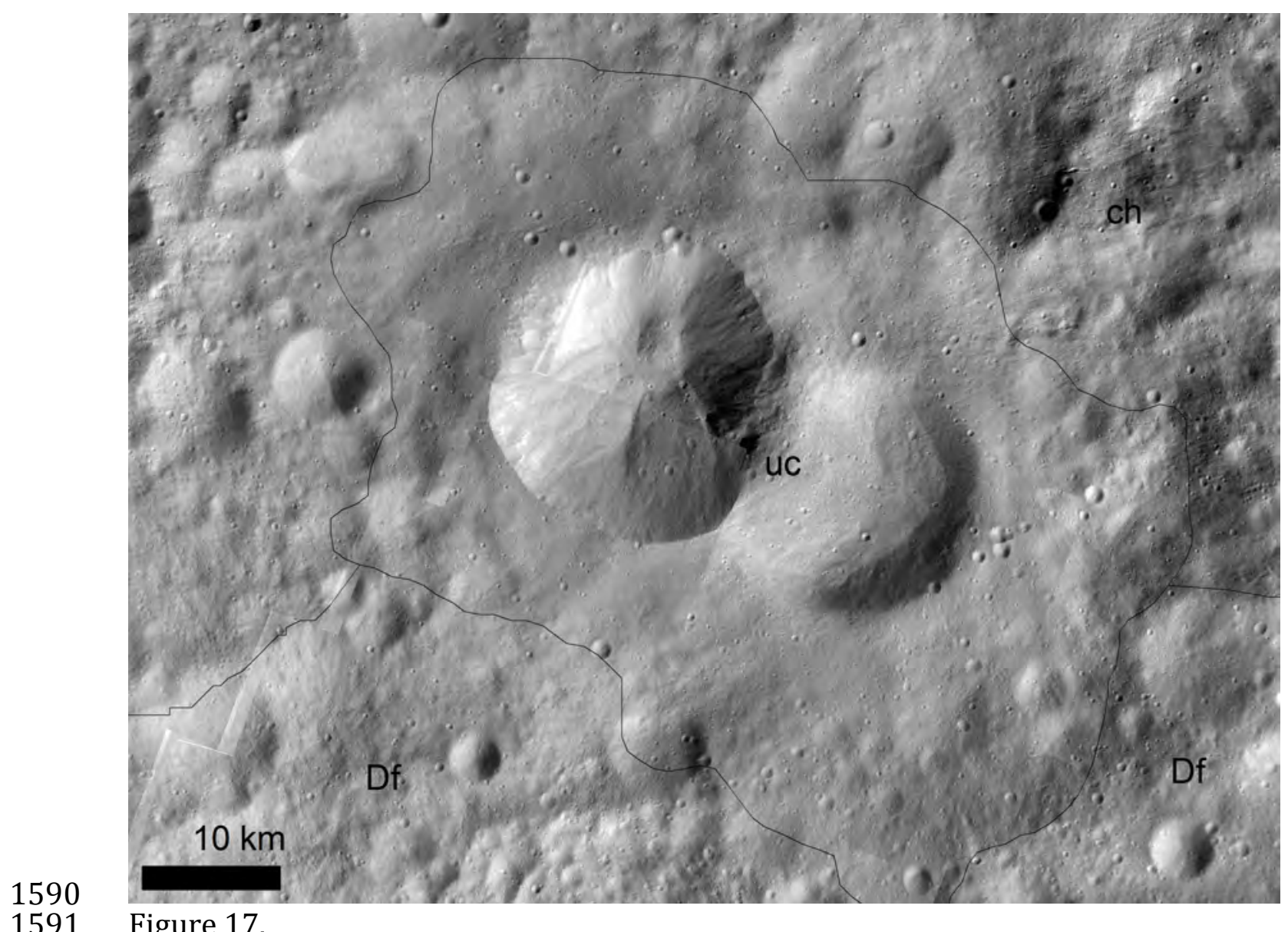

1591 Figure 17. 


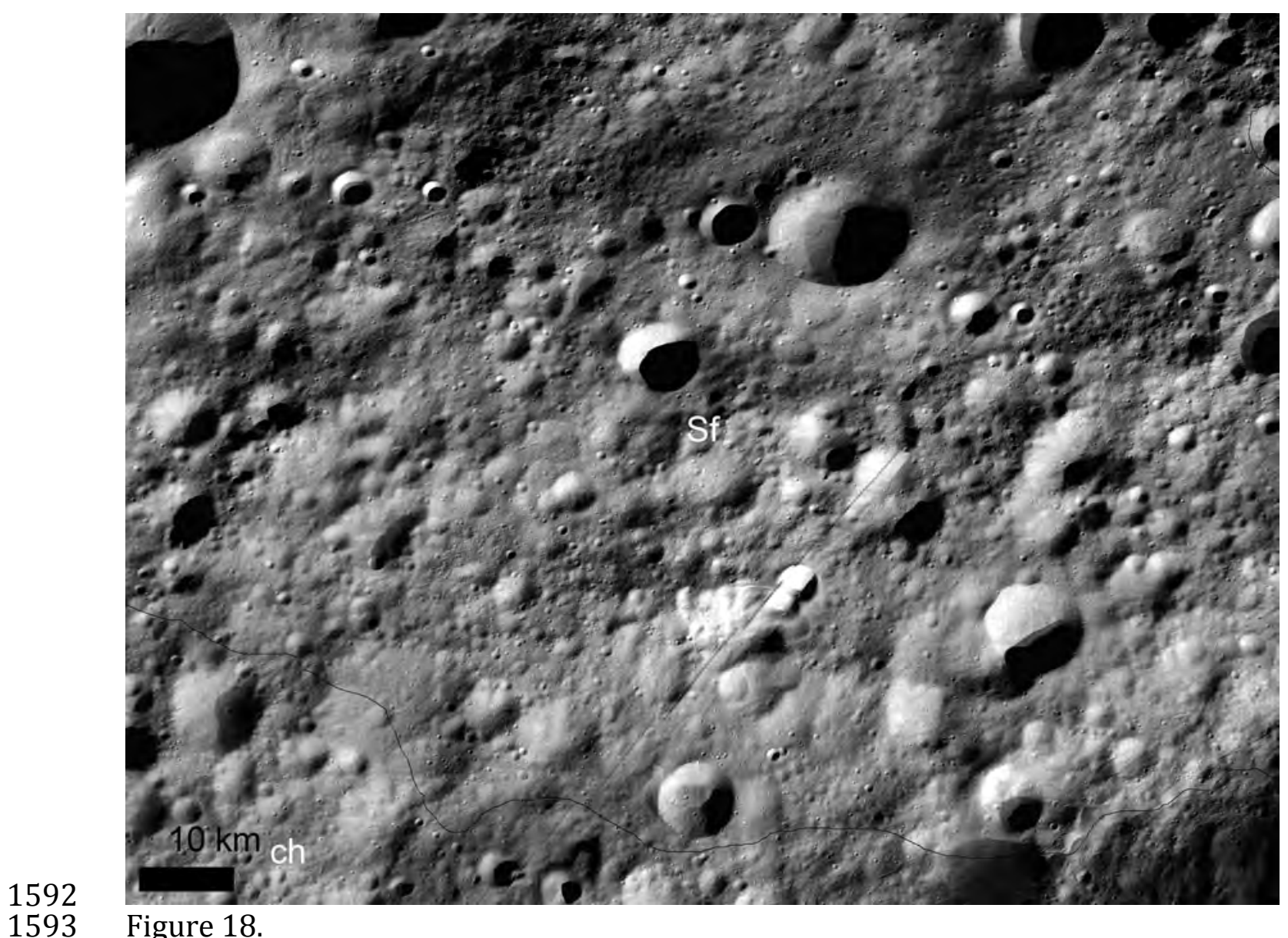

1593 Figure 18. 


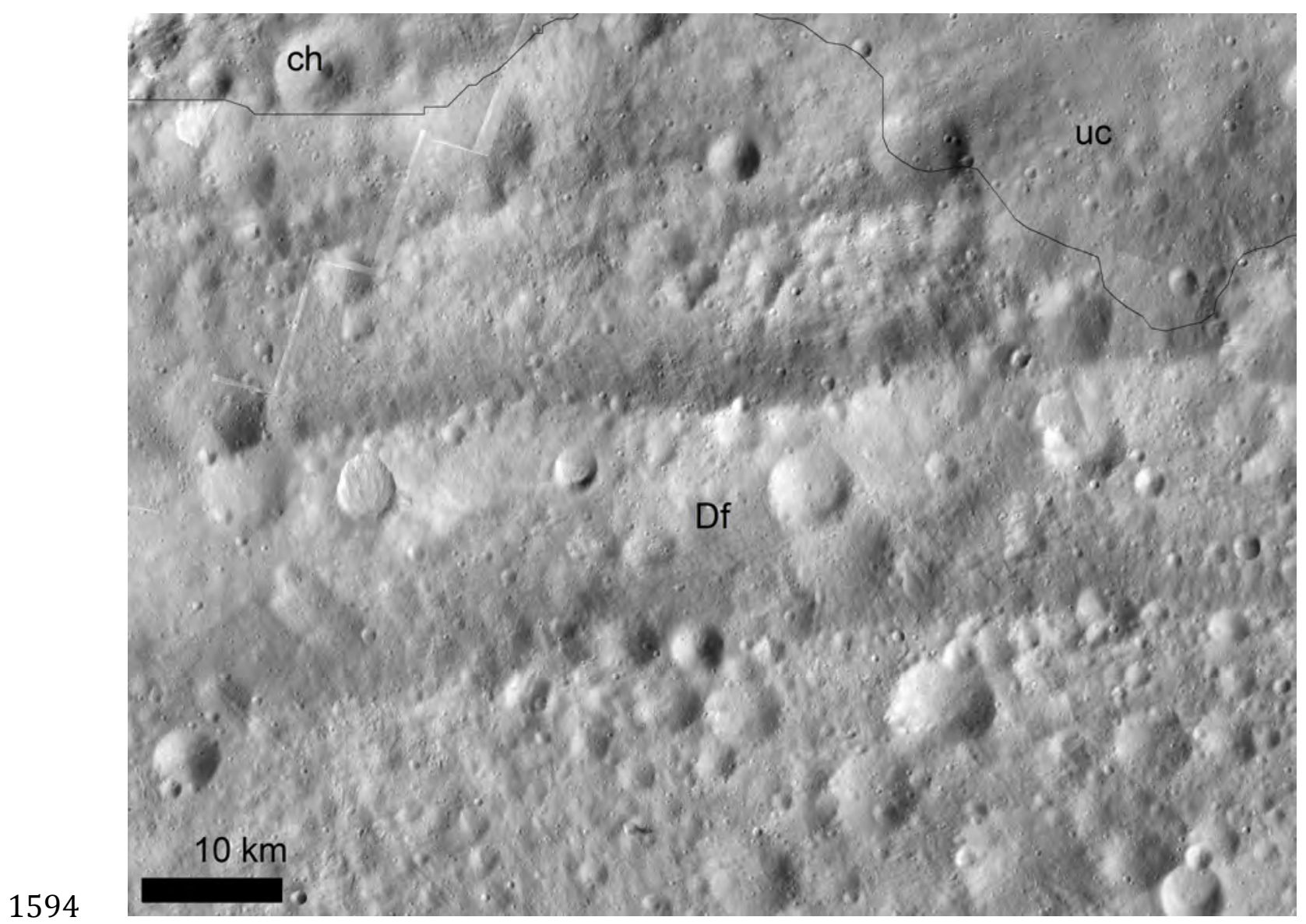

1595 Figure 19. 


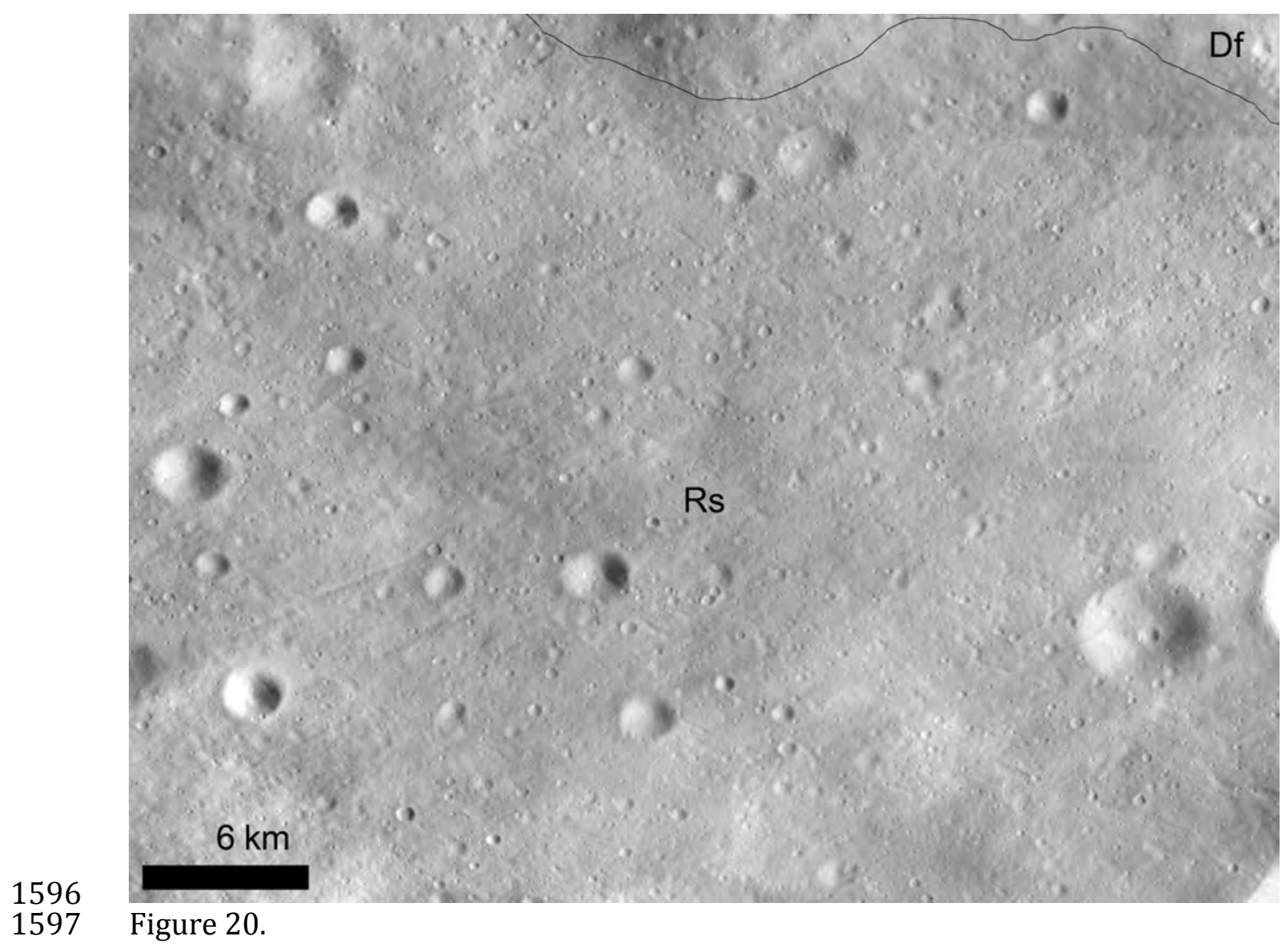




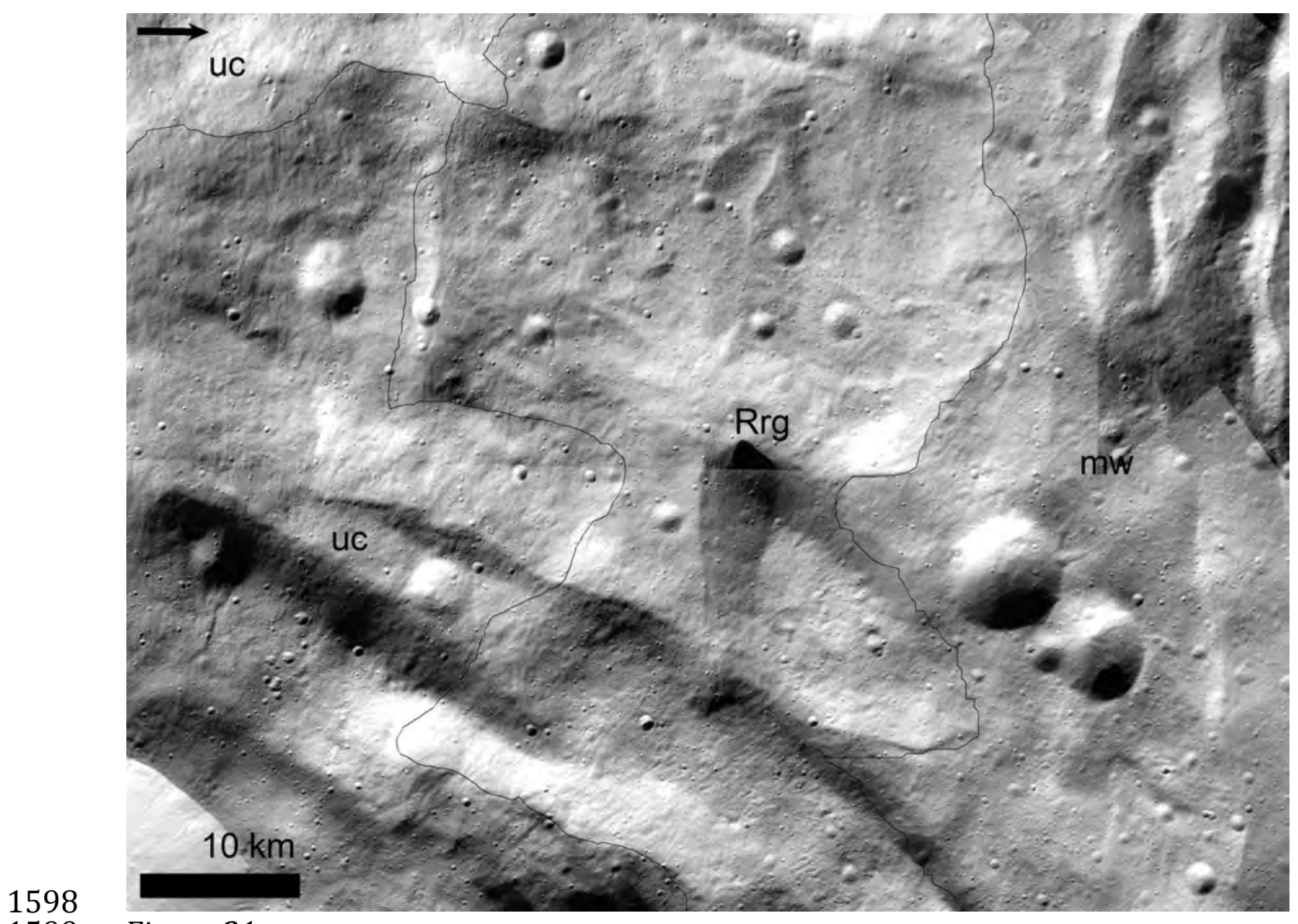

1599 Figure 21. 


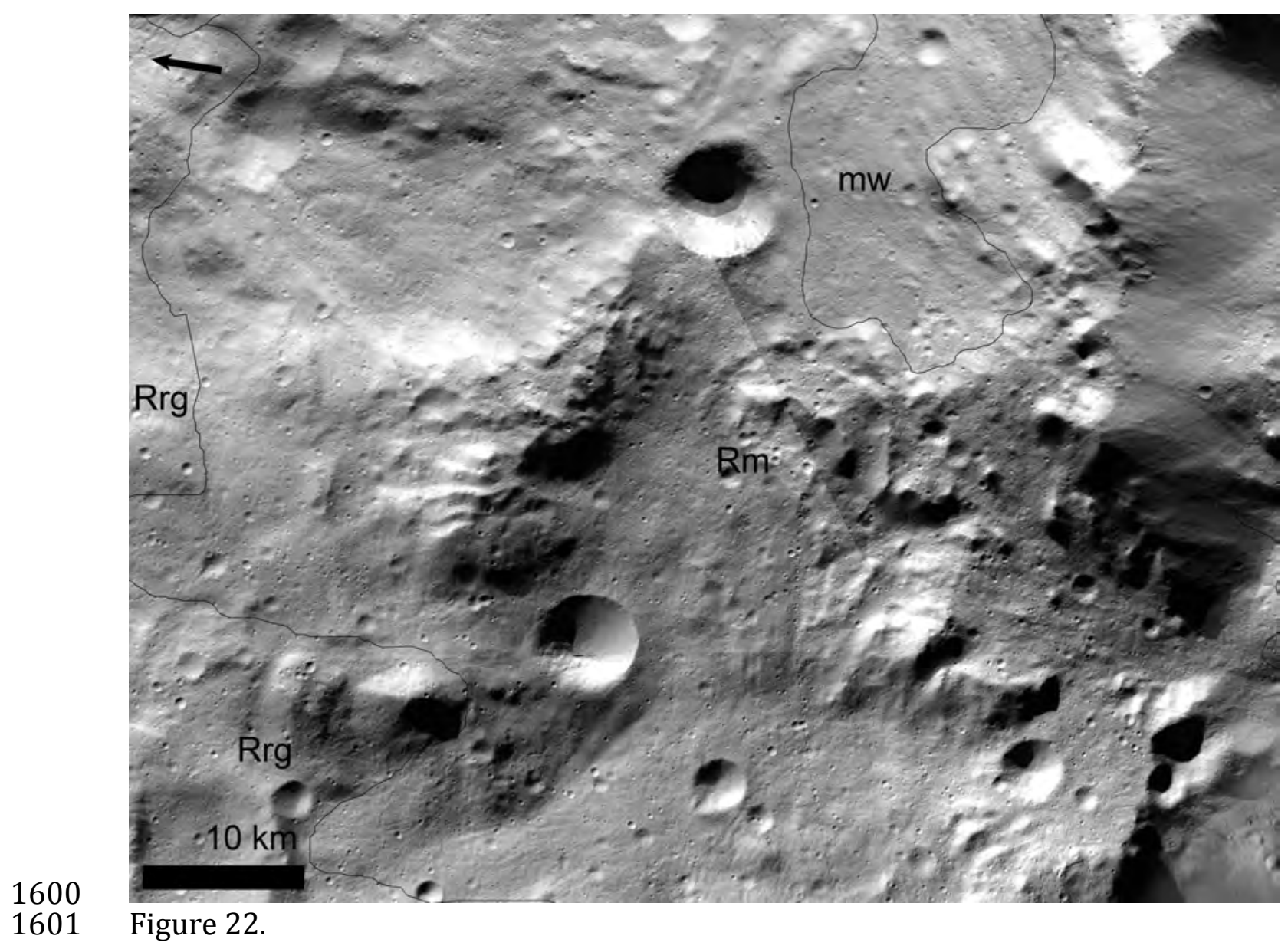



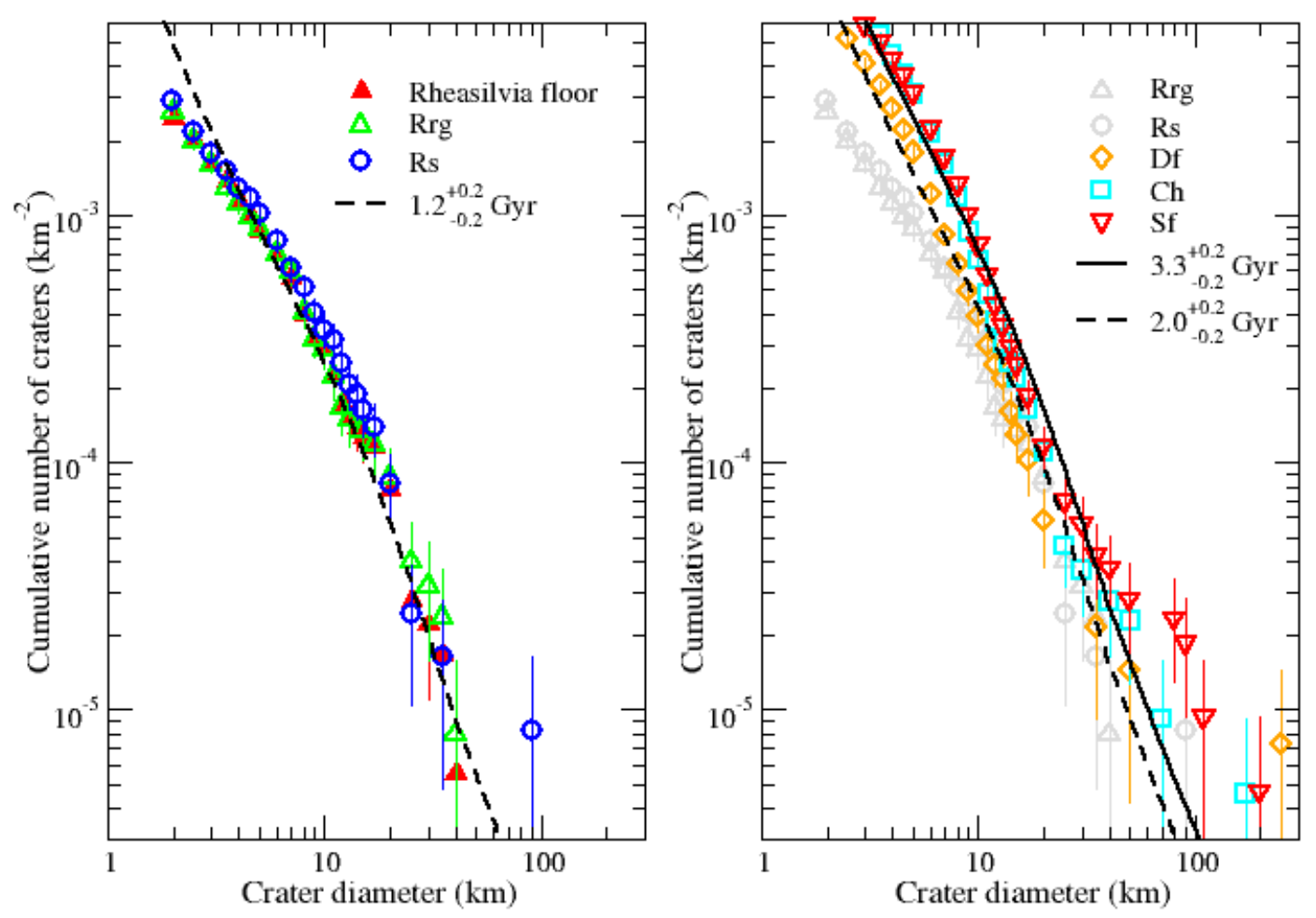

Figure 23. 
cratered highlands (ch)

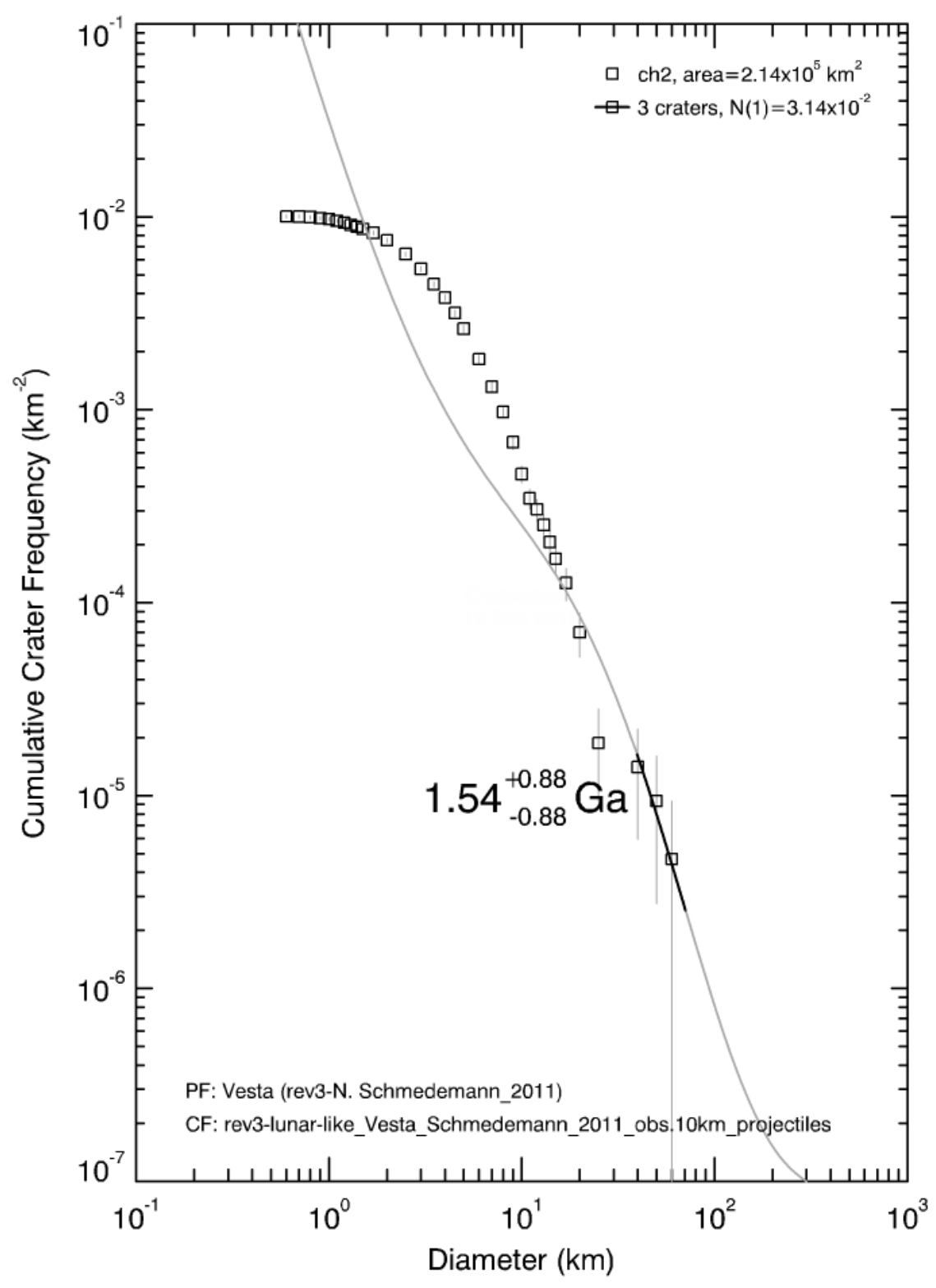

1604

1605

a. 
Saturnalia Fossae unit (Sf)

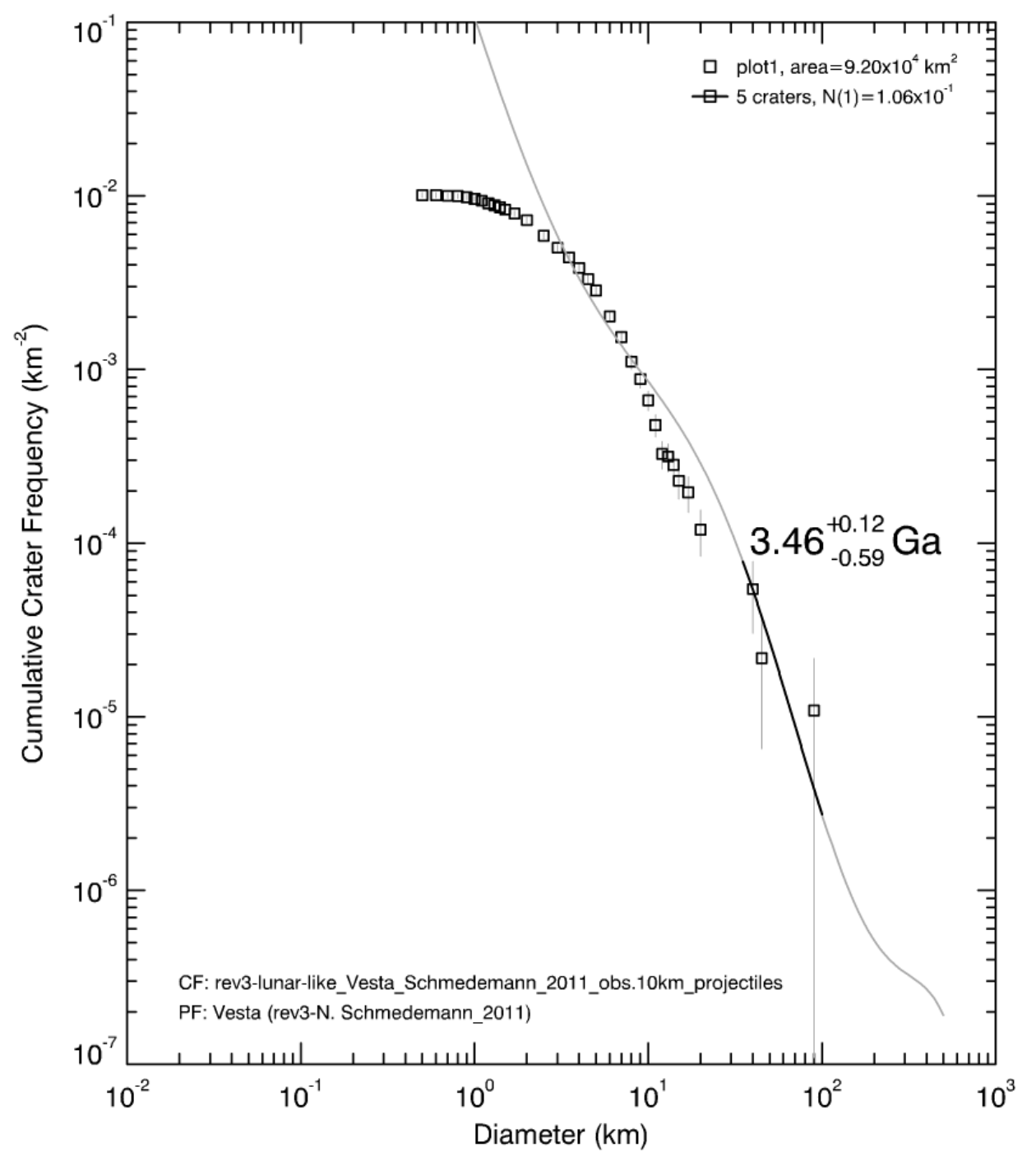

b. 


\section{Divalia Fossae unit (Df)}

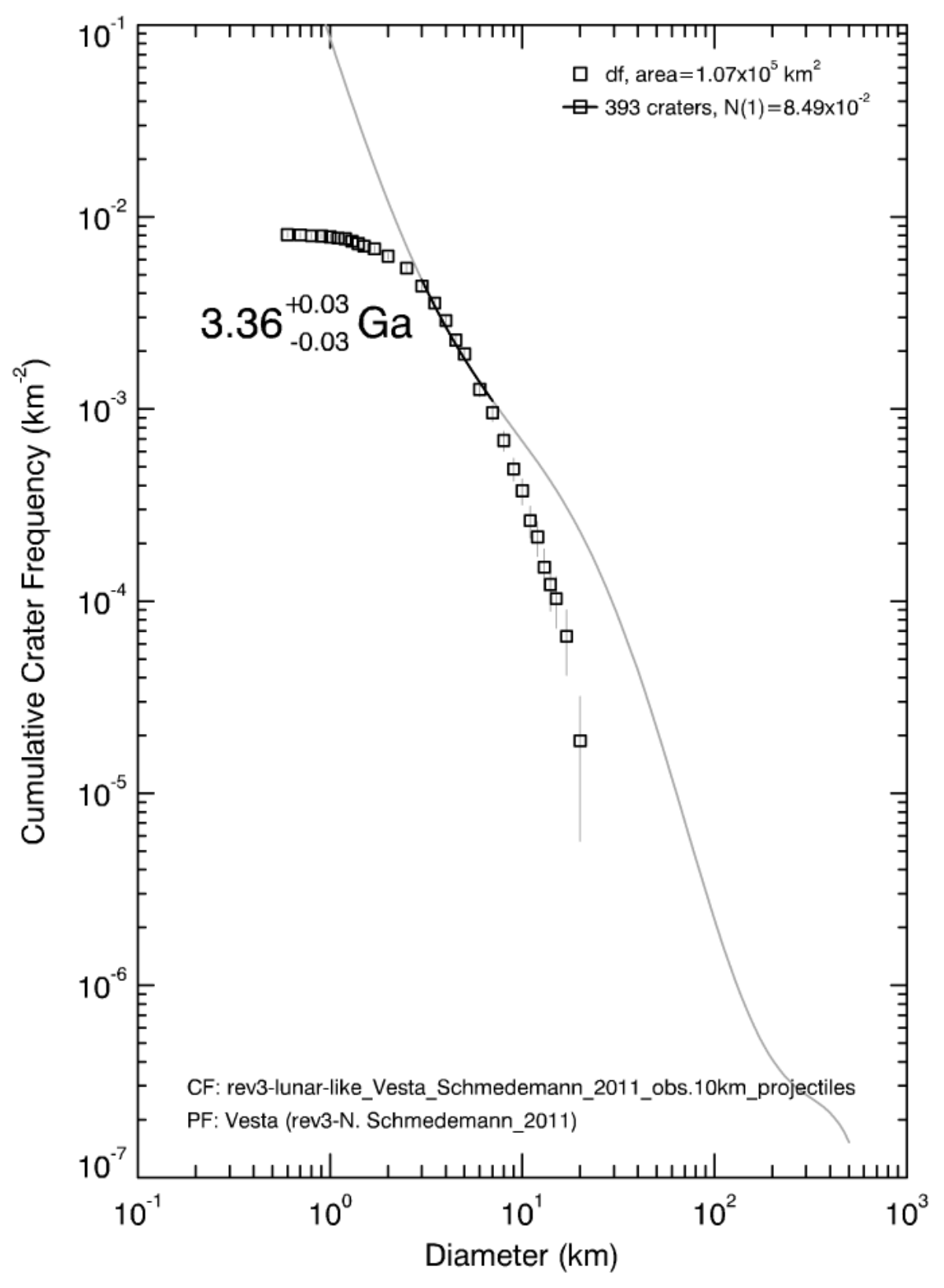


cratered plains (cp)

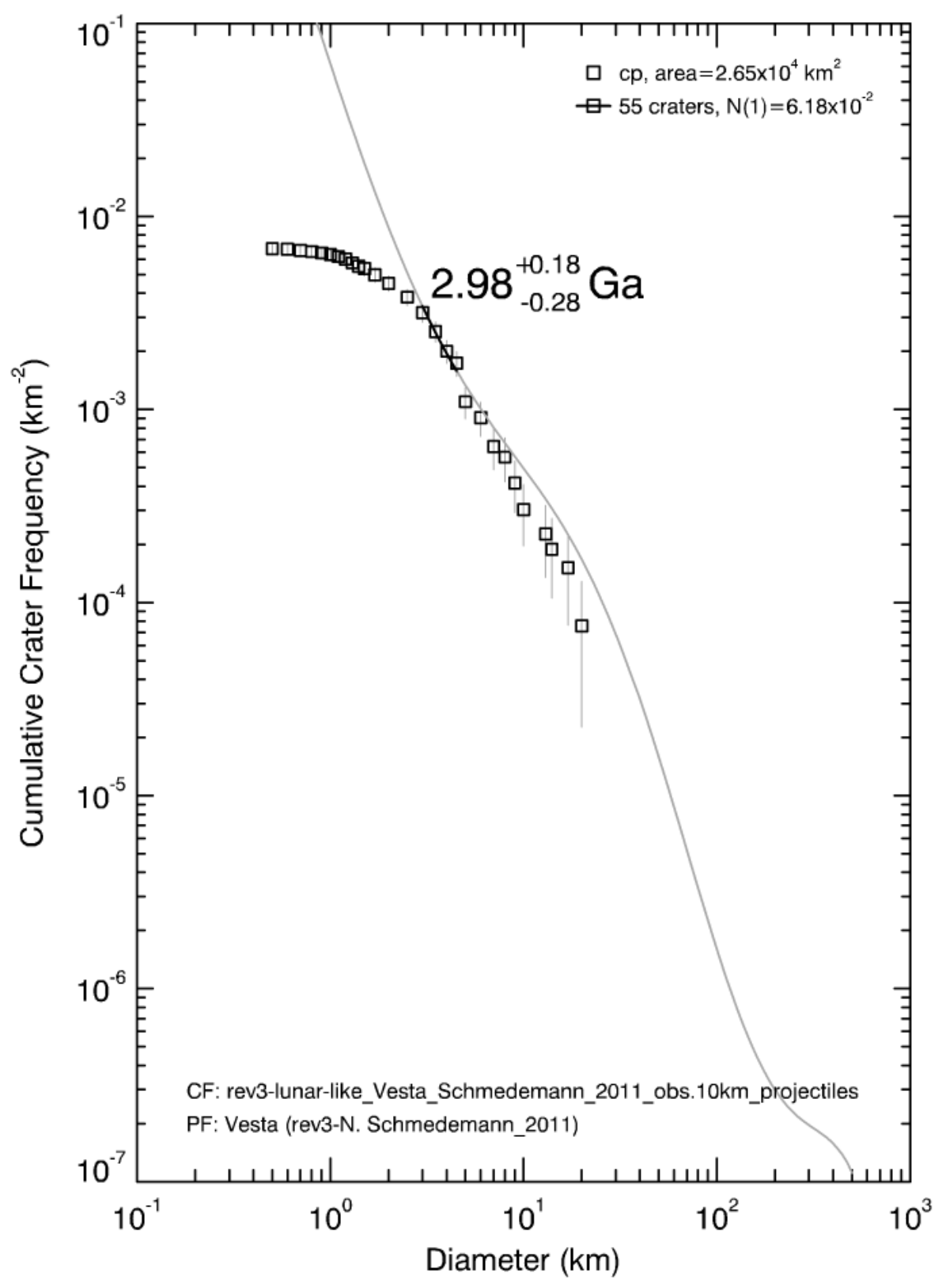

1610

1611

d. 


\section{Rheasilvia smooth terrain (Rs)}

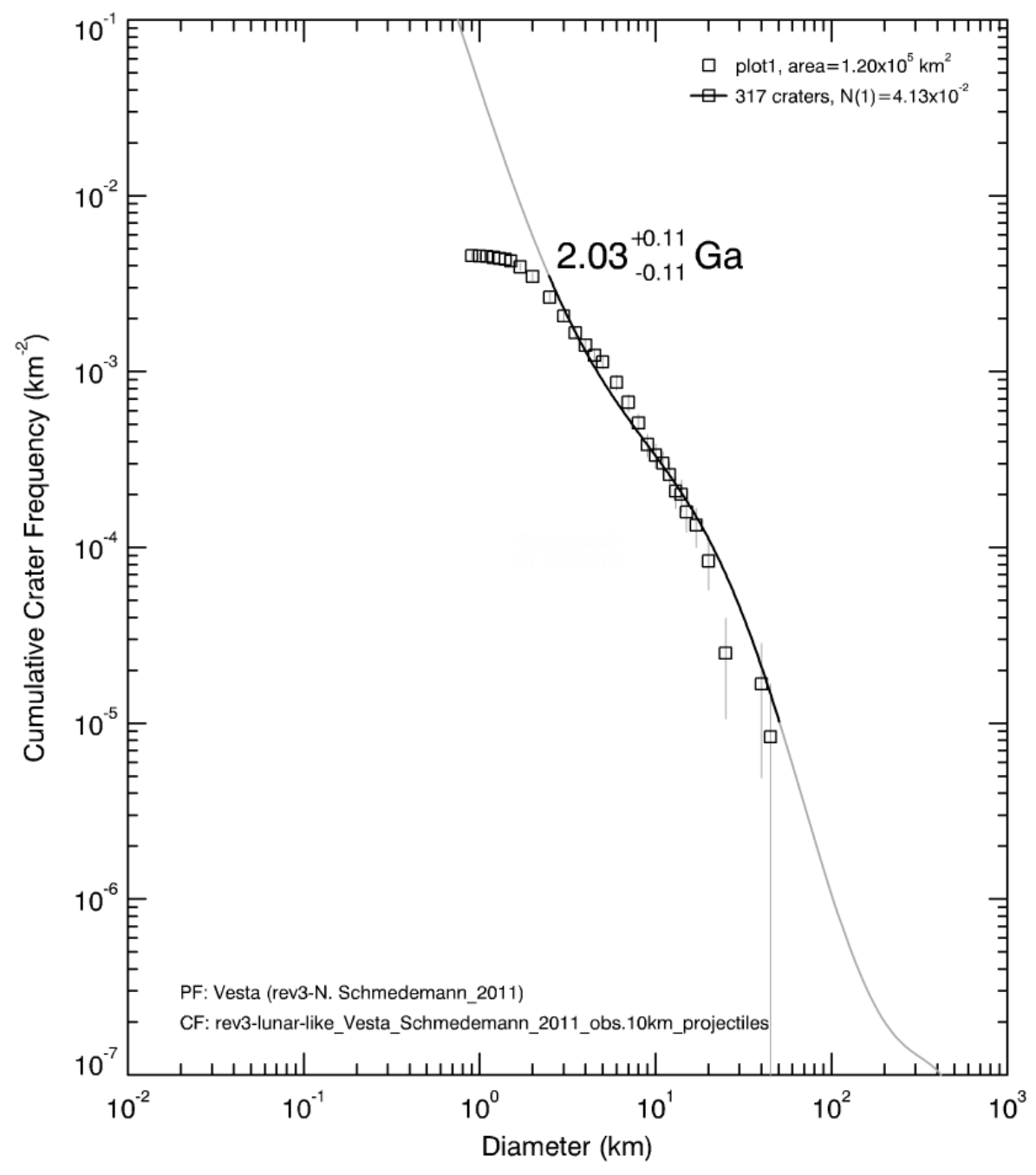




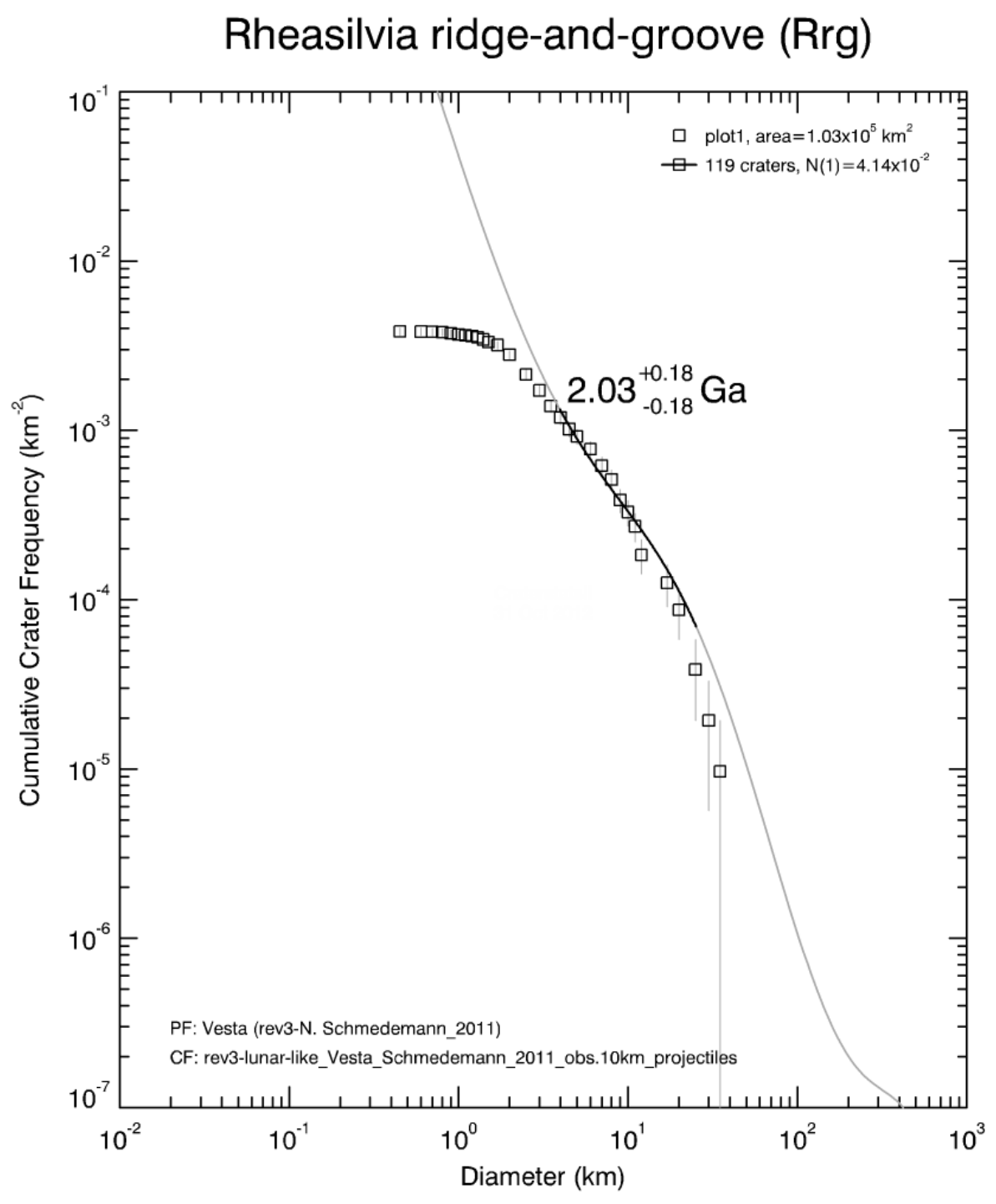

1613 
Rheasilvia mound (Rm)

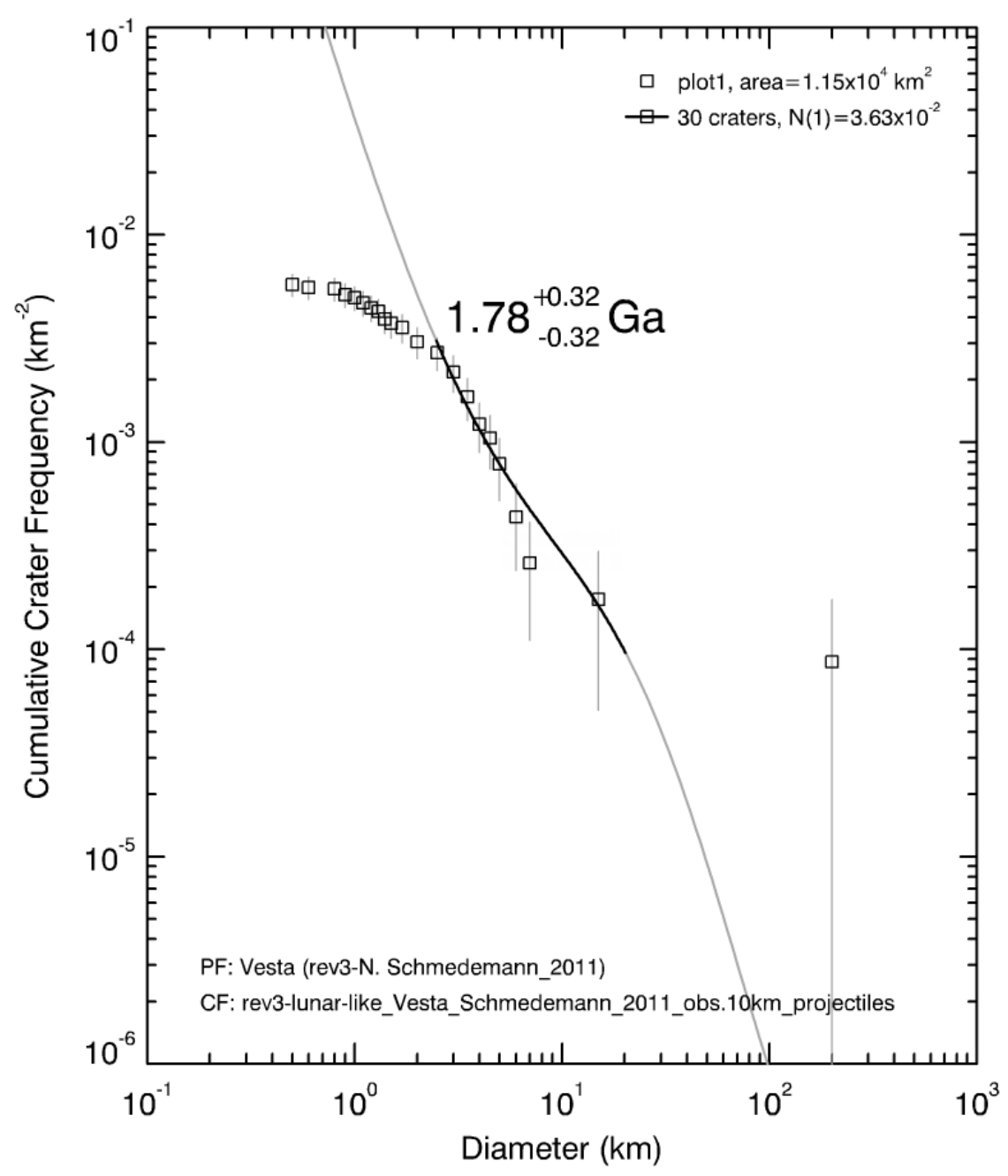

1614

g.

1615 
tholus (t)

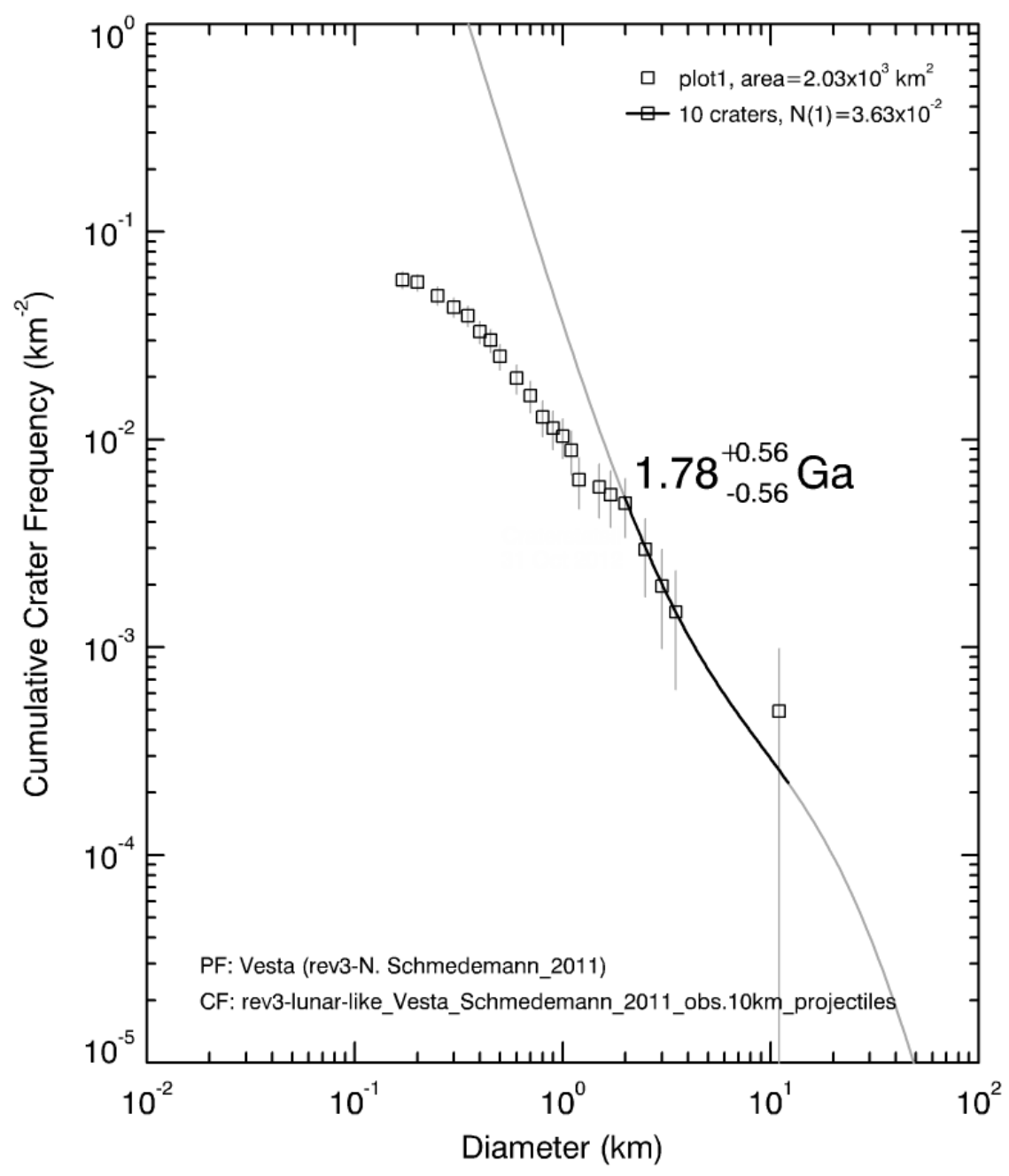

1616

h 


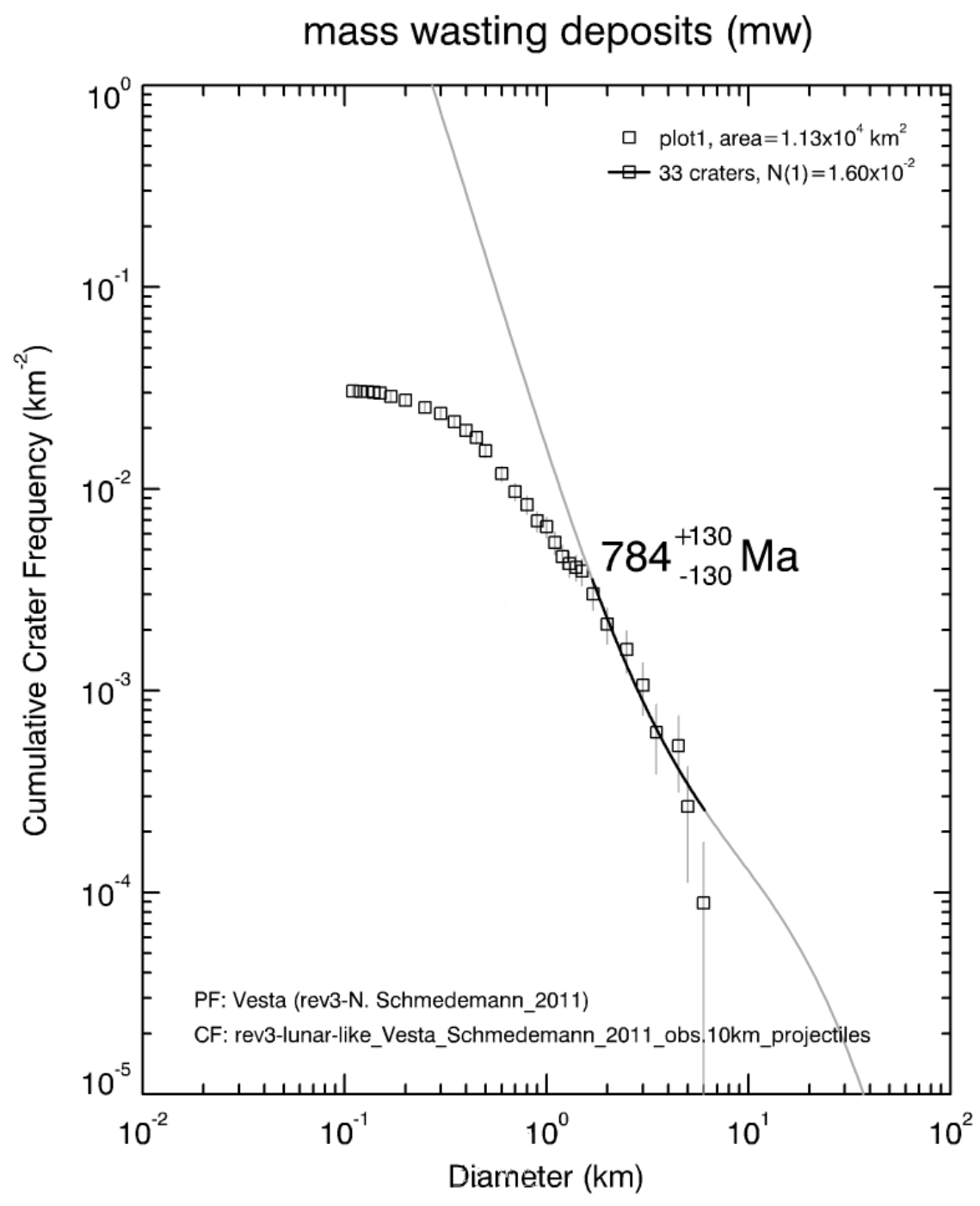

1618 
bright lobate deposits (bl)

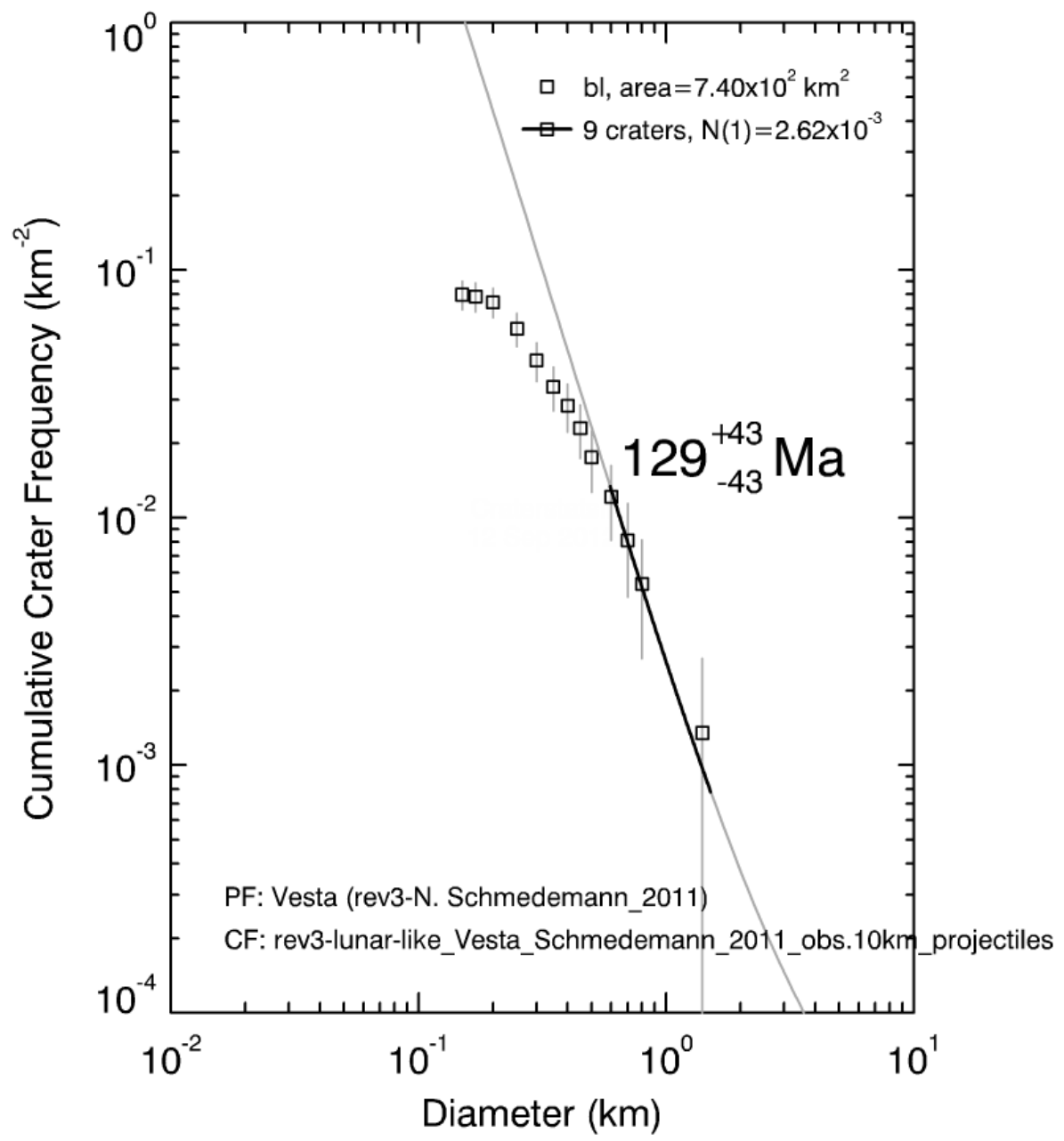

1620 


\section{dark lobate deposits (dl)}

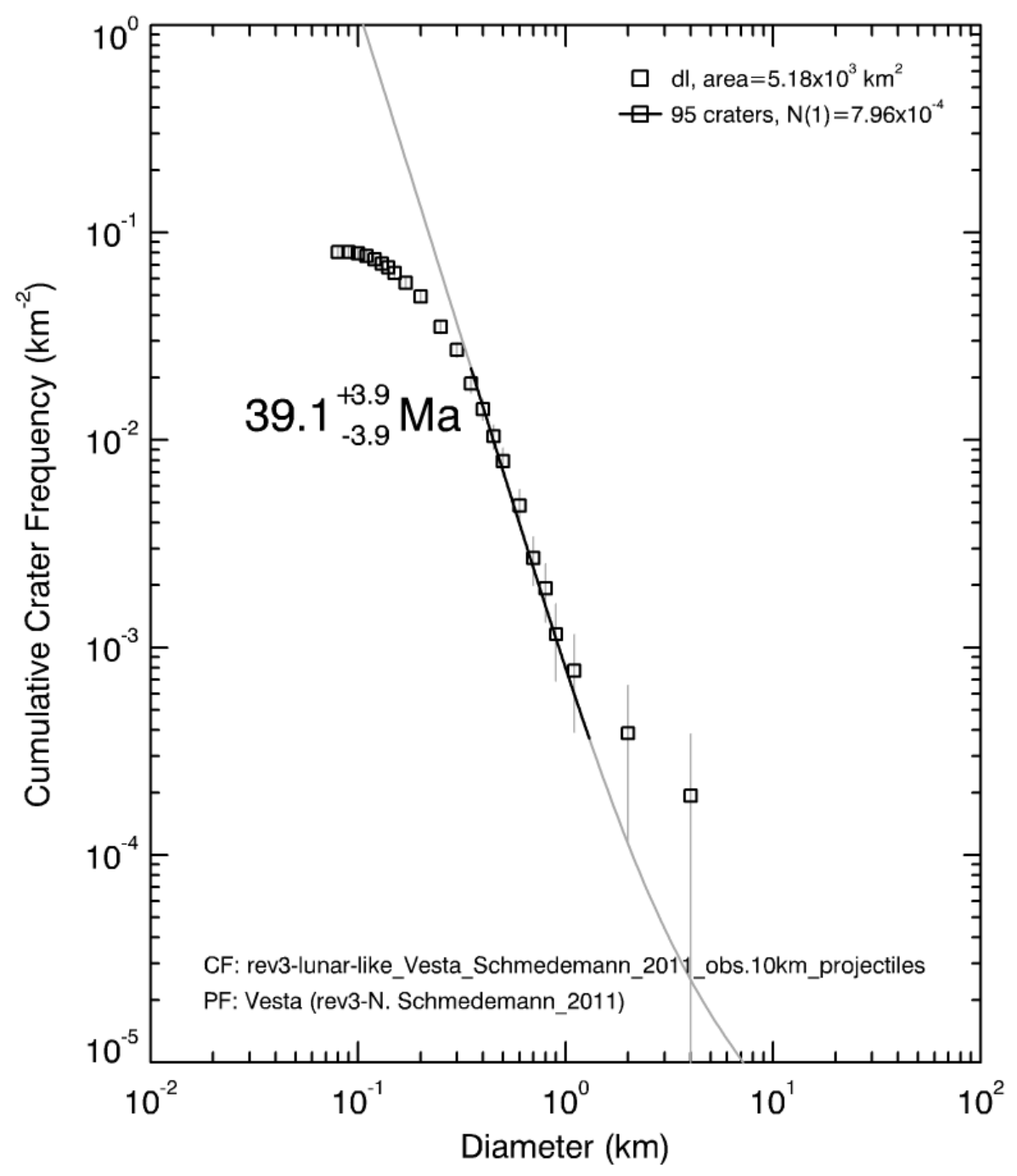

1622

1623

1624

1625

1626

Figure 24.

1627

1628 


\section{CORRELATION OF MAP UNITS}

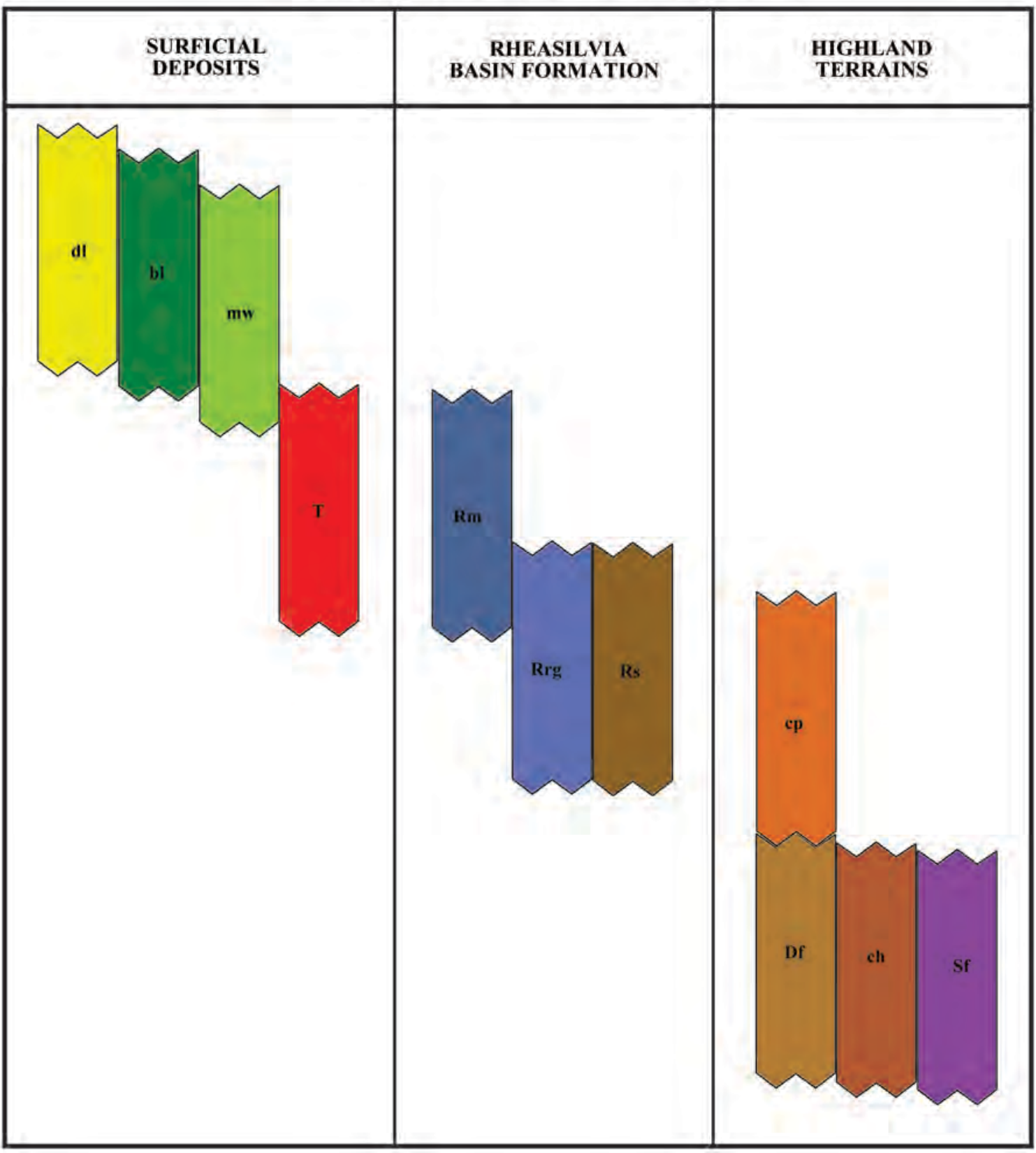

1629

1630

Figure 25.

1631

1632

1633

1634

1635 
1636 Table 1.

1637

\begin{tabular}{|l|c|c|c|}
\hline & RC/OpNav & Survey & HAMO \\
\hline Framing Camera, mosaic & $400 \mathrm{~m} /$ pixel & $250 \mathrm{~m} /$ pixel & $60 \mathrm{~m} / \mathrm{pixel}$ \\
\hline DTM & $750 \mathrm{~m} / \mathrm{pxl}$ & $445 \mathrm{~m} / \mathrm{pxl}$ & $92 \mathrm{~m} / \mathrm{pixel}$ \\
\hline Geologic mapping scale & $1: 20-25 \mathrm{M}$ & $1: 1 \mathrm{M}$ & $1: 500 \mathrm{~K}$ \\
\hline
\end{tabular}

1638 